\title{
24. Low-Temperature Scanning Probe Microscopy
}

\author{
Mehmet Z. Baykara, Markus Morgenstern, Alexander Schwarz, Udo D. Schwarz
}

This chapter is dedicated to scanning probe microscopy (SPM) operated at cryogenic temperatures, where the more fundamental aspects of phenomena important in the fields of nanoscience and nanotechnology can be investigated with high sensitivity under well-defined conditions. In general, scanning probe techniques allow the measurement of physical properties down to the nanometer scale. Some techniques, such as scanning tunneling microscopy (STM) and scanning force microscopy (SFM), even go down to the atomic scale. Various properties are accessible. Most importantly, one can image the arrangement of atoms on conducting surfaces by STM and on insulating samples by SFM. However, electronic states (scanning tunneling spectroscopy), force interaction between different atoms (scanning force spectroscopy), magnetic domains (magnetic force microscopy), magnetic exchange interactions (magnetic exchange force microscopy and spectroscopy), local capacitance (scanning capacitance microscopy), local contact potential differences (Kelvin probe force microscopy), local temperature (scanning thermal microscopy), and local light-induced excitations (scanning near-field microscopy) can also be measured with high spatial resolution, among others. In addition, some modern techniques even allow the controlled manipulation of individual atoms/molecules and the visualization of the internal structure of individual molecules. Moreover, combined STM/SFM experiments are now possible, mainly thanks to the advent of tuning forks as sensing elements in lowtemperature (LT) SPM systems.

Probably the most important advantage associated with the low-temperature operation of scanning probes is that it leads to a significantly better signal-to-noise ratio than measuring at room temperature. This is why many researchers work below $100 \mathrm{~K}$. However, there are also physical reasons to use low-temperature equipment. For example, visualizing the internal structure of molecules with SFM or the utilization of scanning tunneling spectroscopy with high energy resolu- tion can only be realized at low temperatures. Moreover, some physical effects such as superconductivity or the Kondo effect are restricted to low temperatures. Here, we describe the advantages of low-temperature scanning probe operation and the basics of related instrumentation. Additionally, some of the important results achieved by low-temperature scanning probe microscopy are summarized. We first focus on the STM, giving examples of atomic manipulation and the analysis of electronic properties in different material arrangements, among others. Afterwards, we describe results obtained by SFM, reporting on atomic-scale and submolecular imaging, as well as three-dimensional (3-D) force spectroscopy. Results obtained with the method of Kelvin probe force microscopy (KPFM) that is used to study variations in local contact potential difference (LCPD) are briefly discussed. Magnetic force microscopy (MFM), magnetic exchange force microscopy (MExFM), and magnetic resonance force microscopy (MRFM) are also introduced. Although the presented selection of results is far from complete, we feel that it gives an adequate impression of the fascinating possibilities of low-temperature scanning probe instruments.

In this chapter low temperatures are defined as lower than about $100 \mathrm{~K}$ and are normally achieved by cooling with liquid nitrogen or liquid helium. Applications in which SPMs are operated close to $0^{\circ} \mathrm{C}$ are not covered in this chapter.

\subsection{Microscope Operation at Low} Temperatures. 771

24.1.1 Drift .............................................. 771

24.1.2 Noise ........................................ 771

24.1.3 Stability ..................................... 771

24.1.4 Piezo Relaxation and Hysteresis ............ 771

24.2 Instrumentation ........................... 772

24.3 Scanning Tunneling Microscopy and Spectroscopy............................ 773

24.3.1 Atomic Manipulation ..................... 774

24.3.2 High-Resolution Spectroscopy ............ 775

24.3.3 Imaging Electronic Wave Functions ..... 779 
24.3.4 Imaging Spin Polarization: Nanomagnetism

24.4 Scanning Force Microscopy and Spectroscopy

788

24.4.1 Atomic-Scale and Intramolecular Imaging

24.4.2 Force Spectroscopy

24.4.3 Atomic and Molecular Manipulation....

789

790

793
24.4.4 Kelvin Probe Force Microscopy 794

24.4.5 Magnetic Force Microscopy ....

24.4.6 Magnetic Exchange Force Microscopy and Spectroscopy

24.4.7 Magnetic Resonance Force Microscopy.
24.5 Summary...

References.
795

798

799

799

Three decades ago, the first design of an experimental setup was presented where a sharp tip was systematically scanned over a sample surface in order to acquire local information on the tip-sample interaction down to the atomic scale. This original instrument used the tunneling current between a conducting tip and a conducting sample as a feedback signal and was thus named the scanning tunneling microscope [24.1]. Soon after this historic breakthrough, it became widely recognized that virtually any type of tip-sample interaction could be used to obtain local information on the sample by applying the same general principle, provided that the selected interaction was reasonably short-ranged. Thus, a whole variety of new methods has been introduced, which are denoted collectively as scanning probe methods. Overviews are provided in [24.2] and more recently [24.3].

The various methods, especially the above-mentioned scanning tunneling microscopy (STM) and scanning force microscopy (SFM) - which is often further classified into subdisciplines such as topographyreflecting atomic force microscopy (AFM), Kelvin probe force microscopy (KPFM), or magnetic force microscopy (MFM) - have been established as standard methods for surface characterization on the nanometer scale. The reason is that they feature extremely high resolution (often down to the atomic scale for STM and AFM), despite a principally simple, compact, and comparatively inexpensive design.

An important advantage of the simple working principle and the compact design of many scanning probe microscopes (SPMs) is that they can be adapted to different environments such as air, all kinds of gaseous atmospheres, liquids, or vacuum with reasonable effort. Another advantage is their ability to work within a wide temperature range. Microscope operation at higher temperatures is chosen to study surface diffusion, surface reactivity, surface reconstructions that only manifest at elevated temperatures, high-temperature phase transitions, or to simulate conditions as they occur, e.g.,

in engines, catalytic converters or reactors. Ultimately, the upper limit for the operation of an SPM is determined by the stability of the sample. However, thermal drift, which limits the ability to move the tip in a controlled manner with respect to the sample, as well as the depolarization temperature of the piezoelectric positioning elements might further restrict successful measurements.

Conversely, low-temperature (LT) application of SPMs is much more widespread than operation at high temperatures. Essentially five reasons make researchers adapt their experimental setups to low-temperature compatibility. These are:

1. Reduced thermal drift

2. Lower noise levels

3. Enhanced stability of tip and sample

4. Reduction in piezo hysteresis/creep

5. Probably the most obvious, the fact that many physical effects are restricted to low temperature.

Reasons 1-4 only apply unconditionally if the whole microscope body is kept at low temperature (typically by employing a bath cryostat, Sect. 24.2). Conversely, setups in which only the sample or the tip is cooled (typically those that employ flow cryostats) may show considerably less favorable operating characteristics. As a result of $1-4$, ultrahigh resolution and long-term stability can be achieved on a level that significantly exceeds what can be accomplished at room temperature even under the most favorable circumstances. Typical examples of (5) are superconductivity [24.4] and the Kondo effect [24.5].

This chapter is organized such that the advantages associated with low-temperature microscope operation are introduced first, followed by an overview of instrumentational aspects. Subsequently, certain exemplary results of low-temperature STM and SFM operation are presented and the chapter is concluded with a summary. 


\subsection{Microscope Operation at Low Temperatures}

\subsubsection{Drift}

Thermal drift originates from thermally activated movements of individual atoms, which are reflected by the thermal expansion coefficient. At room temperature, typical values for solids are in the order of $(1-50) \times$ $10^{-6} \mathrm{~K}^{-1}$. If the temperature could be kept precisely constant, any thermal drift would vanish, regardless of the absolute temperature of the system. The close coupling of the microscope to a large temperature bath that maintains a constant temperature therefore ensures a significant reduction in thermal drift and allows for distortion-free long-term measurements. As such, microscopes that are efficiently attached to sufficiently large bath cryostats show a one- to two-orderof-magnitude increase in thermal stability compared with nonstabilized setups operated at room temperature.

A second effect also helps suppress thermally induced drift of the probing tip relative to a specific location on the sample surface: The thermal expansion coefficients of materials at liquid-helium temperatures are two or more orders of magnitude smaller than at room temperature. Consequently, thermal drift during low-temperature operation decreases significantly.

For some specific scanning probe methods, there may be additional ways in which a change in temperature can affect the quality of the data. In frequencymodulation SFM (FM-SFM), for example, the measurement principle relies on the accurate determination of the eigenfrequency of the cantilever, which is determined by its spring constant and its effective mass. However, the spring constant changes with temperature due to both thermal expansion (i. e., the resulting change in cantilever dimensions) and the variation of the Young's modulus with temperature. Assuming temperature drift rates of about $2 \mathrm{mK} / \mathrm{min}$, as is typical for room-temperature measurements, this effect might have a significant influence on the obtained data.

It should be mentioned that software-based correction of thermal drift to a certain extent in SPM experiments is now a possibility [24.6,7]. Despite this fact, low-temperature operation currently provides the most direct and reliable route to the elimination of thermal-drift-related effects.

\subsubsection{Noise}

The theoretically achievable resolution in SPM often increases with decreasing temperature due to a decrease in thermally induced noise. An example is the thermal noise in SFM, which is proportional to the square root of the temperature $[24.8,9]$. Lowering the temperature from $T=300$ to $10 \mathrm{~K}$ thus results in a reduction of the thermal frequency noise by more than a factor of five, enabling, e.g., three-dimensional force fields to be acquired with atomic resolution on low-corrugation surfaces such as graphite [24.10].

Another, even more striking, example is the spectroscopic resolution in scanning tunneling spectroscopy (STS). This depends linearly on the temperature [24.2] and is consequently reduced even more at LT than the thermal noise in AFM. This provides the opportunity to study structures or physical effects not accessible at room temperature such as Landau levels in semiconductors [24.11].

Finally, it might be worth mentioning that the enhanced stiffness of most materials at low temperatures (increased Young's modulus) leads to a reduced coupling to external noise. Even though this effect is considered small [24.9], it should not be ignored, especially when combined with the fact that artifacts in atomic-resolution SPM experiments due to tip elasticity $[24.12,13]$ are also expected to decrease at low temperatures due to the increase in stiffness.

\subsubsection{Stability}

There are two major stability issues that considerably improve at low temperature. First, low temperatures close to the temperature of liquid helium inhibit most of the thermally activated diffusion processes. As a consequence, the sample surfaces show a significantly increased long-term stability since defect motion and/or adatom diffusion is massively suppressed. Most strikingly, even single xenon atoms deposited on suitable substrates can be successfully imaged $[24.14,15]$ or even manipulated [24.16]. In the same way, low temperatures also stabilize the atomic configuration at the tip end by preventing sudden jumps of the most loosely bound, foremost tip atom(s). Moreover, the large cryostat that usually surrounds the microscope acts as an effective cryo-pump. Thus, samples can be kept clean for several weeks, which is a multiple of the corresponding time at room temperature (about 3-4h).

\subsubsection{Piezo Relaxation and Hysteresis}

The last important benefit of low-temperature operation of SPMs is that artifacts from the response of the piezoelectric scanners are substantially reduced. After applying a voltage ramp to one electrode of a piezoelectric scanner, its immediate initial deflection, $l_{0}$, is followed by a much slower relaxation, $\Delta l$, with a loga- 
rithmic time dependence. This effect, known as piezo relaxation or creep, diminishes substantially at low temperatures, typically by a factor of ten or more.
As a consequence, piezo nonlinearities and piezo hysteresis decrease accordingly. Additional information is given in [24.17].

\subsection{Instrumentation}

The two main design criteria for all ultrahigh vacuum (UHV)-based SPM systems (which we are exclusively focusing on) are: (1) to provide an efficient decoupling of the microscope from the UHV system and other sources of external vibrations, and (2) to avoid most internal noise sources through the high mechanical rigidity of the microscope body itself. In vacuum systems designed for low-temperature applications, a significant degree of complexity is added, since, on the one hand, close thermal contact of the SPM and cryogen is necessary to ensure the (nearly) drift-free conditions described above, while, on the other hand, good vibration isolation (both from the outside world, as well as from the boiling or flowing cryogen) has to be maintained.

During the last couple of decades, a considerable number of both home-built and commercial SPM designs have been presented for low-temperature operation. Because of the variety of different approaches, it is not possible to cover all related aspects here. Instead, we will briefly discuss a home-built combined STM/SFM system that is specifically geared toward atomic-resolution imaging and spectroscopy at low temperatures that relies on a bath cryostat [24.18] rather than a flow cryostat. The main advantage of using bath cryostats instead of flow cryostats for low-temperature operation is the fact that the whole microscope is kept at the same cryogenic temperature which thus (nearly) eliminates the occurrence of thermal drift between the tip and the sample.

Drawings of the (i) UHV system that houses the specific microscope to be discussed here, (ii) the cryostat to which the microscope is attached, and (iii) the microscope itself, are provided in Fig. 24.1. The UHV system (Fig. 24.1a) is partially based on a commercial design by Omicron Nanotechnology (Germany) and features two individual chambers for sample preparation (via sputtering, annealing, oxygen plasma deposition, etc.) and surface analysis (via conventional surface science techniques such as low-energy electron diffraction (LEED) and Auger spectroscopy) as well as a third chamber (the SPM chamber) that houses the microscope attached to the on-top bath cryostat. Samples are introduced into the UHV system via a fast entry load lock pumped by a turbo pump and sample transfer between different chambers occurs in situ via magnetic transfer arms. All chambers are equipped with ion get-

ter and titanium sublimation pumps that allow base pressures on the order of $10^{-11} \mathrm{mbar}$ to be reached. Additionally, the SPM and preparation chambers are equipped with turbo pumps for the bakeout process and pump-down from ambient pressure. For vibration isolation purposes, the whole UHV system is situated on 12 active vibration isolation units in a sound-proofed room on a basement floor.

The bath cryostat employed in the present design (Fig. 24.1b) has been fabricated by Cryovac and consists of a central dewar for liquid helium with a capacity of 8.51 and a shielding dewar that can contain up to 181 of liquid nitrogen. This setup allows low liquid helium consumption $(\approx 31 /$ day $)$. The cryostat features, on the vacuum chamber side, a double set of thermal radiation shields (gold-coated copper) that are cooled by the liquid helium and liquid nitrogen dewars, respectively. The microscope is situated inside the thermal shields and may be suspended on a set of three springs for vibrational isolation from the cryostat during measurements. Thanks to a set of sliding doors on the shields, sample and tip transfer occur in situ (as the microscope is cold and under vacuum) and cool-down times after transfer are on the order of $2 \mathrm{~h}$. During operation, the whole microscope is at a typical temperature of $\approx 5 \mathrm{~K}$.

The microscope itself (Fig. 24.1c) allows combined STM/SFM operation thanks to the fact that it employs quartz tuning forks rather than the conventional micromachined SFM cantilevers. While the particular details associated with using tuning forks as sensing elements for atomic-resolution SFM (often combined with STM) are explained elsewhere [24.19], the main advantages involve (i) self-sensing thanks to the piezoelectric character of the quartz and (ii) enhanced sensitivity to short-range forces due to high mechanical stiffness. The main body of the microscope is manufactured out of Macor and is cylindrical with a height of $80 \mathrm{~mm}$ and a diameter of $40 \mathrm{~mm}$. To minimize thermal drift, a (mostly) symmetric structural design has been employed and most metallic parts are made out of titanium which has a similar thermal expansion coefficient to Macor. In this particular design, the tip is stationary and for the coarse approach, the sample is moved vertically with respect to the tip by using a pan-style motor that is based on a sapphire prism in point-contact with several shear-piezo-stacks (see [24.18] for details). 


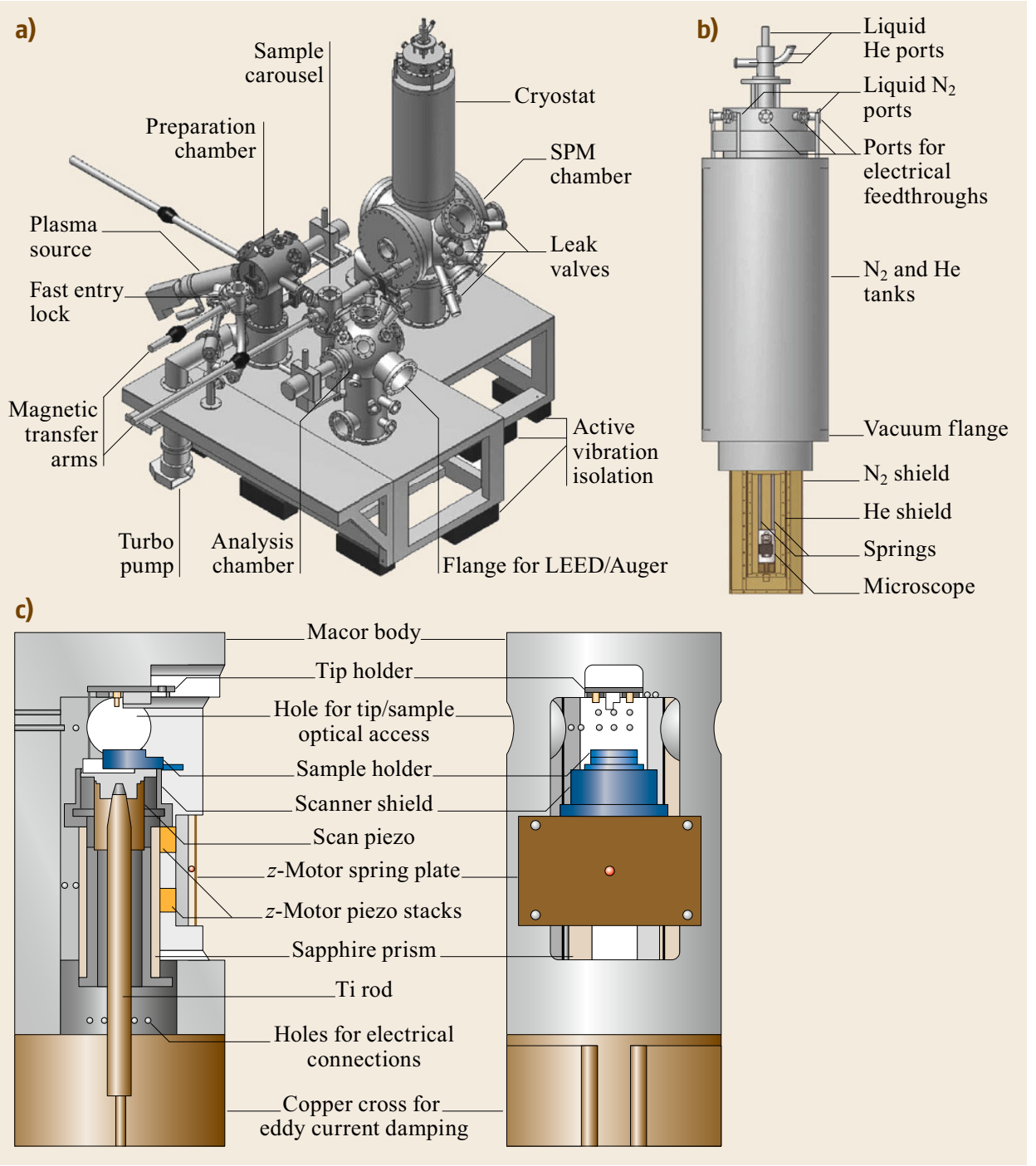

Fig. 24.1 Schematic drawings of the (a) UHV system that houses the low-temperature microscope described in this chapter, (b) the cryostat to which the microscope is attached and (c) the microscope itself (left: section view, right: front view). Adapted from [24.18]

For precise three-dimensional positioning of the sample with respect to the tip during the fine approach and data acquisition, a piezoelectric element in the shape of a cylinder (the scan piezo) is employed.

To conclude this section, one should re-iterate that careful instrumentational design for low-temperature scanning probe microscopy allows imaging and spectroscopy experiments with ultrahigh resolution and stability to be successfully performed. In particular, the
3-D force spectroscopy data on surface-oxidized copper and titanium dioxide surfaces discussed in Sect. 24.4 have been obtained with the specific microscope presented here. Finally, rapidly increasing prices for liquid helium have recently led to the development of cryogen-free designs for low-temperature SPM with promising results (e.g., by RHK technology). It is projected that this trend in SPM instrumentation will accelerate in the near future.

\subsection{Scanning Tunneling Microscopy and Spectroscopy}

In this section, we review some of the most important results achieved by low-temperature scanning tunneling microscopy (LT-STM). After summarizing the results, placing emphasis on the necessity of LT equipment, we turn to the details of the different experiments and the physical meaning of the results obtained. 
As described in Sect. 24.1, the LT equipment has basically three advantages for scanning tunneling microscopy (STM) and spectroscopy (STS): First, the instruments are much more stable with respect to thermal drift and the coupling to external noise, allowing the establishment of new functionalities for the instrument. In particular, LT-STM has been used to move atoms on a surface [24.16], cut molecules into pieces [24.20], reform bonds [24.21], charge individual atoms [24.22], and, consequently, establish new structures on the nanometer scale. Also, the detection of light resulting from tunneling into a particular molecule [24.23, 24], the visualization of thermally induced atomic movements [24.25], and the detection of hysteresis curves of individual atoms [24.26] require LT instrumentation. More recent accomplishments achieved with LT-STM include the measurement of electron spin relaxation times of individual atoms [24.27] and the detection of magnetic remanence in single atoms [24.28].

Second, the spectroscopic resolution in STS depends linearly on temperature and is, therefore, considerably reduced at LT. This provides the opportunity to study physical effects inaccessible at room temperature. Examples are the resolution of spin and Landau levels in semiconductors [24.11], or the investigation of lifetime-broadening effects on the nanometer scale [24.29]. Also the imaging of distinct electronic wavefunctions in real space requires LT-STM [24.30]. More recently, vibrational levels, spin-flip excitations and phonons have been detected with high spatial resolution at LT using the additional inelastic tunneling channel [24.31-33].

Third, many physical effects, in particular effects guided by electronic correlations, are restricted to low temperature. Typical examples are superconductivity [24.4], the Kondo effect [24.5], and many of the electron phases found in semiconductors [24.34]. Here, LT-STM provides the possibility to study electronic effects on a local scale, and intensive work has been done in this field, the most elaborate with respect to hightemperature superconductivity [24.35-37].

\subsubsection{Atomic Manipulation}

Although manipulation of surfaces on the atomic scale can be achieved at room temperature [24.38,39], only the use of LT-STM allows the placement of individual atoms at desired atomic positions [24.40]. The main reason is that rotation, diffusion, or charge transfer of entities could be excited at higher temperature, making the intentionally produced configurations unstable.

The usual technique to manipulate atoms is to increase the current above a certain atom, which reduces the tip-atom distance, then to move the tip with the atom to a desired position and finally to reduce the current again in order to decouple atom and tip. The first demonstration of this technique was performed by Eigler and Schweizer [24.16], who used Xe atoms on a $\mathrm{Ni}(110)$ surface to write the three letters IBM (their employer) on the atomic scale (Fig. 24.2a). Nowadays, many laboratories are able to move different kinds of atoms and molecules on different surfaces with high precision. An example featuring $\mathrm{CO}$ molecules on $\mathrm{Cu}(110)$ is shown in Fig. 24.2b-g. Even more complex structures than the "2000" shown in the figure, such as cascades of $\mathrm{CO}$ molecules that by mutual repulsive interaction mimic different kinds of logic gates, have been assembled and their functionality tested [24.41]. Although these devices are slow and restricted to low temperature, they nicely demonstrate the high degree of control achieved on the atomic scale.

The basic modes of controlled motion of atoms and molecules by the tip are pushing, pulling, and sliding. The selection of the particular mode depends on the tunneling current, i.e., the distance between tip and molecule, as well as on the particular moleculesubstrate combination [24.42]. It has been shown experimentally that the potential landscape for the adsorbate movement is modified by the presence of the tip [24.43, 44] and that excitations induced by the tunneling current can trigger atomic or molecular motion [24.45, 46]. Other sources of motion are the electric field between tip and molecule or electromigration caused by the high current density [24.40]. The required lateral tip force for atomic motion has been measured for typical adsorbate-substrate combinations to be on the order of $0.1 \mathrm{nN}$ [24.47]. Other types of manipulation on the atomic scale are feasible. Some of them require selective inelastic tunneling into vibrational or rotational modes of the molecules [24.48]. This leads to controlled desorption [24.49], diffusion [24.50], molecular rotation [24.51, 52], conformational change [24.53], or even the controlled pick-up of molecules by the tip [24.21]. Dissociation can be achieved by voltage pulses [24.20] inducing local heating, even if the pulse is applied at distances of $100 \mathrm{~nm}$ away from the molecule [24.54]. Also, association of individual molecules [24.21,5557] can require voltage pulses in order to overcome local energy barriers. The process of controlled bond formation can even be used for doping of single $\mathrm{C}_{60}$ molecules by up to four potassium atoms [24.58]. As an example of controlled manipulation, Fig. $24.2 \mathrm{~h}-\mathrm{m}$ shows the production of biphenyl from two iodobenzene molecules [24.59]. The iodine is abstracted by voltage pulses (Fig. 24.2i,j), then the iodine is moved to the terrace by the pulling mode (Fig. 24.2k,1), and finally the two phenyl parts are slid along the step edge until they are close enough to react (Fig. 24.2m). The 
a)
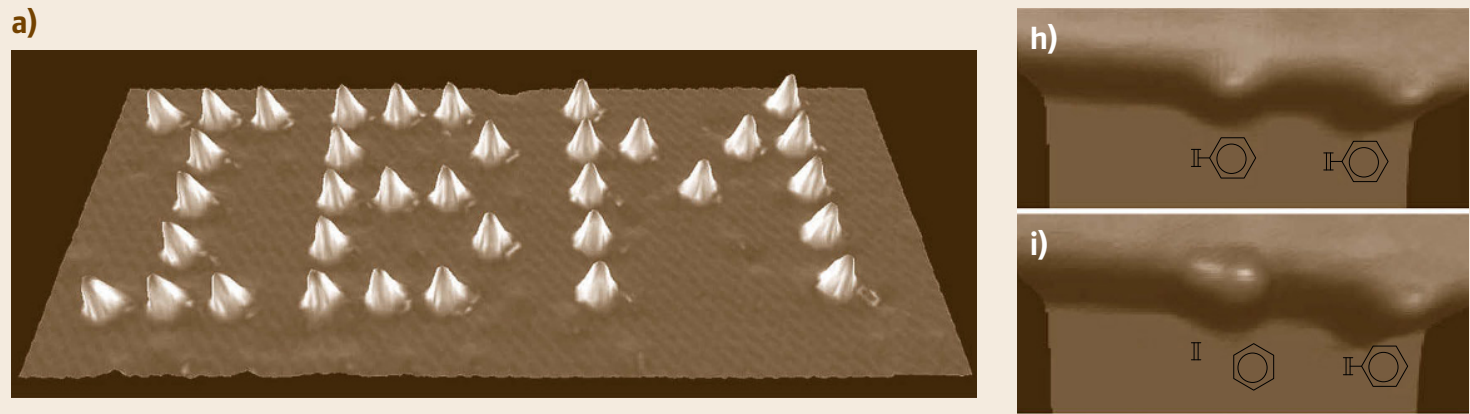

b)

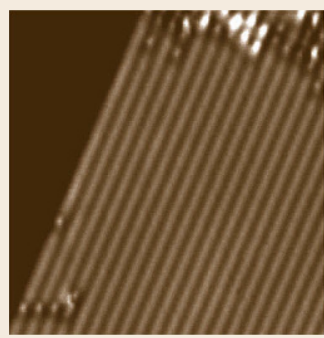

e)

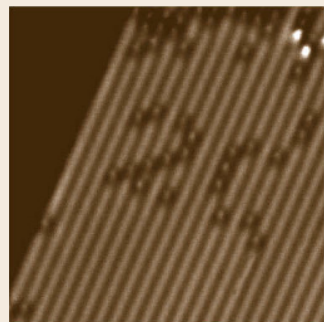

c)

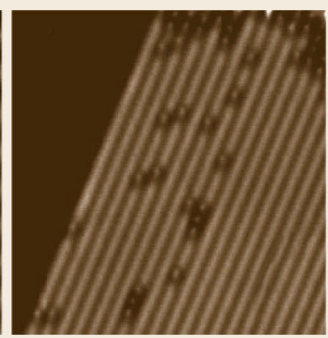

f)

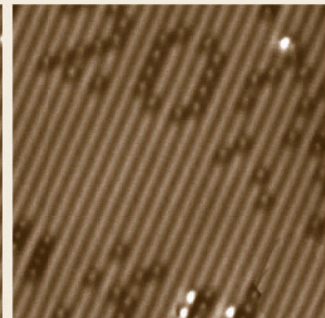

d)

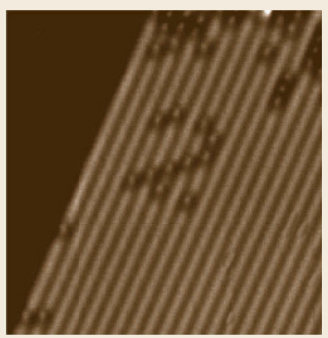

g)

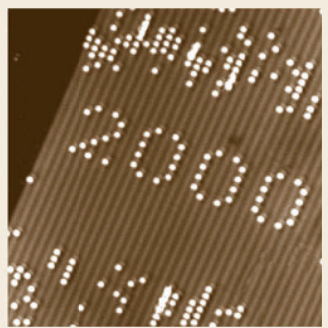

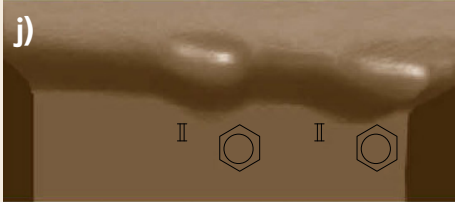
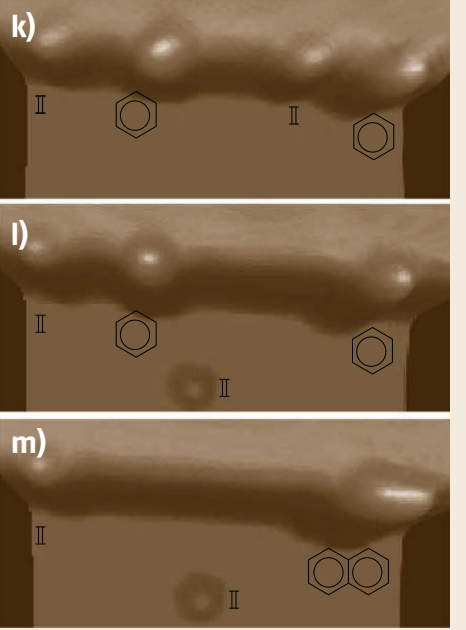

Fig. 24.2 (a) STM image of single Xe atoms positioned on a Ni(110) surface in order to realize the letters $I B M$ on the atomic scale (@ D. Eigler, Almaden); (b-f) STM images recorded after different positioning processes of CO molecules on a $\mathrm{Cu}(110)$ surface; (g) final artwork greeting the new millennium on the atomic scale ((b-g) $\odot$ G. Meyer, Zürich). (h-m) Synthesis of biphenyl from two iodobenzene molecules on $\mathrm{Cu}(111)$ : First, iodine is abstracted from both molecules $(i, j)$; then the iodine between the two phenyl groups is removed from the step $(\mathbf{k})$, and finally one of the phenyls is slid along the $\mathrm{Cu}$ step (I) until it reacts with the other phenyl $(\mathrm{m})$; the line drawings symbolize the actual status of the molecules $((\mathbf{h}-\mathbf{m})$ ) $\mathrm{S}$. W. Hla and K. H. Rieder, Berlin)

chemical identification of the product is not straightforward and partly requires detailed vibrational STM spectroscopy [24.60].

Finally, also the charge state of a single atom or molecule can be manipulated, tested, and read out. $\mathrm{A} \mathrm{Au}$ atom has been switched reversibly between two charge states using an insulating thin film as the substrate [24.22]. In addition, the carrier capture rate of a single impurity level within the bandgap of a semiconductor has been quantified [24.61], and the point conductance of a single atom has been measured and turned out to be a reproducible quantity [24.62]. These promising results might trigger a novel electronic field of manipulation of matter on the atomic scale, which is tightly related to the currently very popular field of molecular electronics.

\subsubsection{High-Resolution Spectroscopy}

One of the most important modes of LT-STM is STS, which detects the differential conductivity $\mathrm{d} I / \mathrm{d} V$ as a function of the applied voltage $V$ and the position $(x, y)$. The $\mathrm{d} I / \mathrm{d} V$ signal is basically proportional to the local density of states (LDOS) of the sample, the sum 
over squared single particle wavefunctions $\Psi_{i}$ [24.2],

$$
\frac{\mathrm{d} I}{\mathrm{~d} V}(V, x, y) \propto \operatorname{LDOS}(E, x, y)=\sum_{\Delta E}\left|\Psi_{i}(E, x, y)\right|^{2},
$$

where $\Delta E$ is the energy resolution of the experiment. In simple terms, each state corresponds to a tunneling channel, if it is located between the Fermi levels $\left(E_{\mathrm{F}}\right)$ of the tip and the sample. Thus, all states located in this energy interval contribute to $I$, while $\mathrm{d} I / \mathrm{d} V(V)$ detects only the states at the energy $E$ corresponding to $V$. The local intensity of each channel depends further on the LDOS of the state at the corresponding surface position and its decay length into vacuum. For s-like tip states, Tersoff and Hamann have shown that it is simply proportional to the LDOS at the position of the tip [24.63]. Therefore, as long as the decay length is spatially constant, one measures the LDOS at the surface (24.1). Note that the contributing states are not only surface states, but also bulk states. However, surface states usually dominate if present. Chen has shown that higher orbital tip states lead to the so-called derivation rule [24.64]: $\mathrm{p}_{\mathrm{z}}$-type tip states detect $\mathrm{d}(\mathrm{LDOS}) / \mathrm{d} z$, $\mathrm{d}_{\mathrm{z}}^{2}$-states detect $\mathrm{d}^{2}$ (LDOS) $/ \mathrm{d} z^{2}$, and so on. As long as the decay into vacuum is exponential and spatially constant, this leads only to an additional, spatially constant factor in $\mathrm{d} I / \mathrm{d} V$. Thus, it is still the LDOS that is measured (24.1). The requirement of a spatially constant decay is usually fulfilled on larger length scales, but not on the atomic scale [24.64]. There, states located close to the atoms show a stronger decay into vacuum than the less localized states in the interstitial region. This effect can lead to STS corrugations that are larger than the real LDOS corrugations [24.65].

The voltage dependence of $\mathrm{d} I / \mathrm{d} V$ is sensitive to a changing decay length with $V$, which increases with $V$. This influence can be reduced at higher $V$ by displaying $\mathrm{d} I / \mathrm{d} V /(I / V)$ [24.66]. Additionally, $\mathrm{d} I / \mathrm{d} V(V)$ curves might be influenced by possible structures in the density of states (DOS) of the tip, which also contribute to the number of tunneling channels [24.67]. However, these structures can usually be identified, and only tips free of characteristic DOS structures are used for quantitative experiments.

Importantly, the energy resolution $\Delta E$ is largely determined by temperature. It is defined as the smallest energy distance of two $\delta$-peaks in the LDOS that can still be resolved as two individual peaks in $\mathrm{d} I / \mathrm{d} V(V)$ curves and is $\Delta E=3.3 \mathrm{k}_{\mathrm{B}} \mathrm{T}$ [24.2]. The temperature dependence is nicely demonstrated in Fig. 24.3, where the tunneling gap of the superconductor $\mathrm{Nb}$ is measured at different temperatures [24.68]. The peaks at the rim

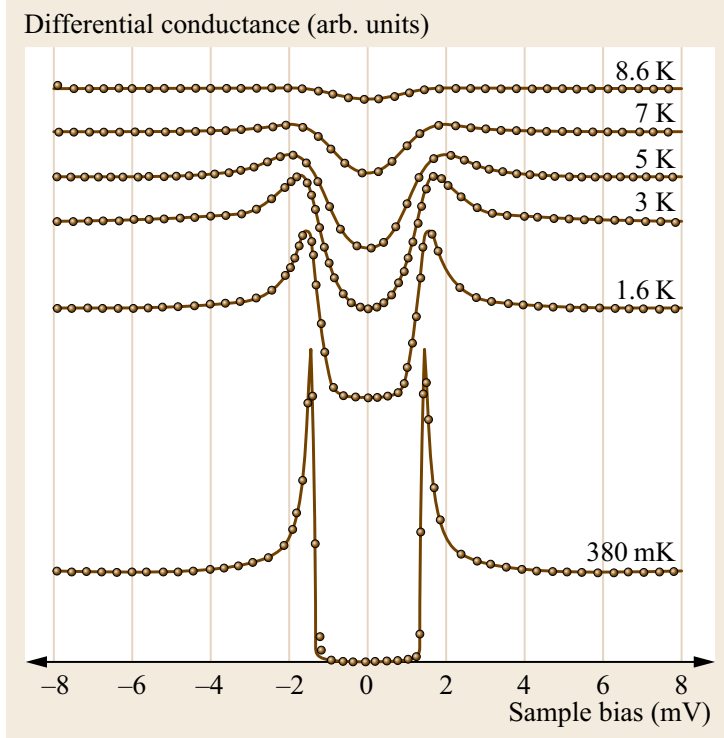

Fig. 24.3 Differential conductivity curve $\mathrm{d} I / \mathrm{d} V(V)$ measured on a Au surface by a Nb tip (circles). Different temperatures are indicated; the lines are fits according to the superconducting gap of $\mathrm{Nb}$ folded with the temperaturebroadened Fermi distribution of the $\mathrm{Au}$ (C) S.H. Pan, Houston)

of the gap get wider at temperatures well below the critical temperature of the superconductor $\left(T_{\mathrm{c}}=9.2 \mathrm{~K}\right)$.

\section{Lifetime Broadening}

Besides $\Delta E$, intrinsic properties of the sample lead to a broadening of spectroscopic features. Basically, the finite lifetime of the electron or hole in the corresponding state broadens its energetic width. Any kind of interaction such as electron-electron interaction can be responsible. Lifetime broadening has usually been measured by photoemission spectroscopy (PES), but it turned out that lifetimes of surface states on noblemetal surfaces determined by STS (Fig. 24.4a,b) are up to a factor of three larger than those measured by PES [24.69]. The reason is probably that defects broaden the PES spectrum. Defects are unavoidable in a spatially integrating technique such as PES, thus STS has the advantage of choosing a particularly clean area for lifetime measurements. The STS results can be successfully compared with theory, highlighting the dominating influence of intraband transitions for the surface-state lifetime on $\mathrm{Au}(111)$ and $\mathrm{Cu}(111)$, at least close to the onset of the surface band [24.29].

With respect to band electrons, the analysis of the width of the band onset on $\mathrm{d} I / \mathrm{d} V(V)$ curves has the disadvantage of being restricted to the onset energy. Another method circumvents this problem by mea- 


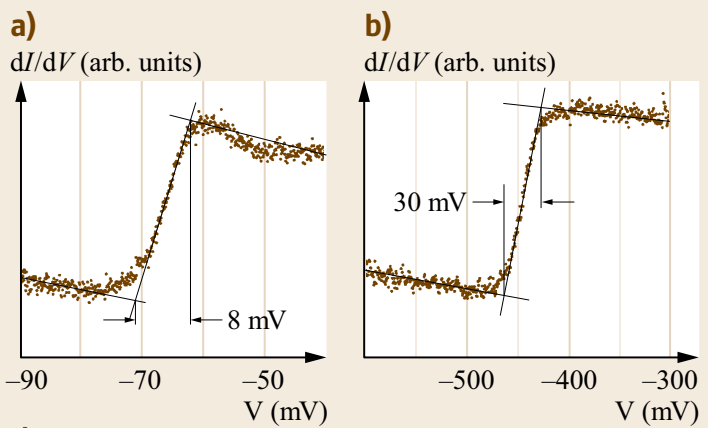

c)

$\mathrm{d} / / \mathrm{d} V$ (arb. units)

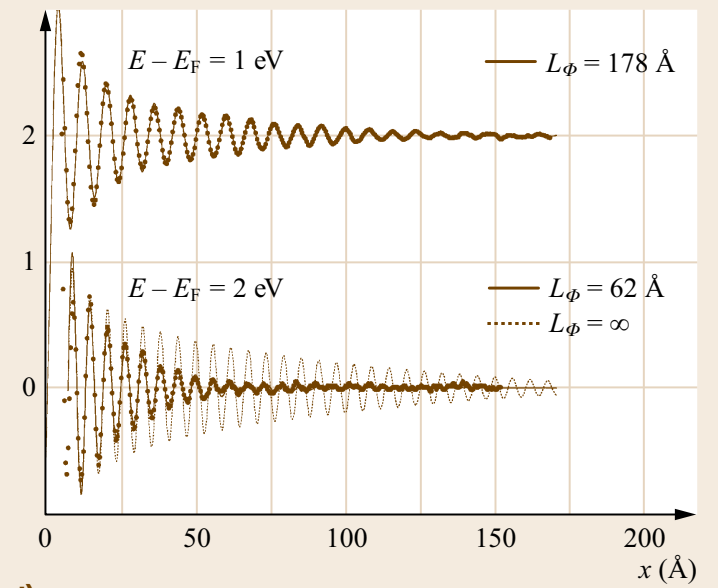

d)

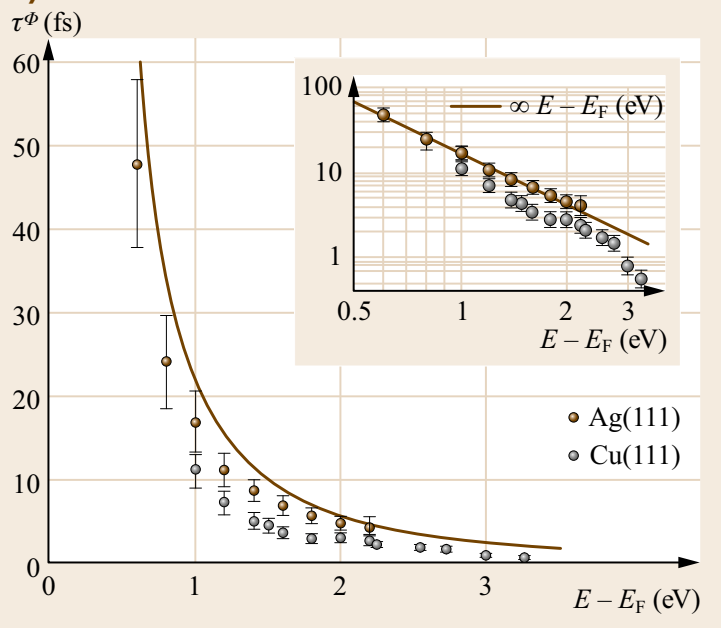

suring the decay of standing electron waves scattered from a step edge as a function of energy [24.70]. Figure $24.4 \mathrm{c}, \mathrm{d}$ shows the resulting oscillating $\mathrm{d} I / \mathrm{d} V$ signal measured for two different energies. To deduce the coherence length $L_{\Phi}$, which is inversely proportional to the lifetime $\tau_{\Phi}$, one has to consider that the finite energy resolution $\Delta E$ in the experiment also
Fig. $24.4(\mathrm{a}, \mathrm{b})$ Spatially averaged $\mathrm{d} I / \mathrm{d} V$ curves of $\mathrm{Ag}(111)$ and $\mathrm{Cu}(111)$; both surfaces exhibit a surface state with parabolic dispersion, starting at -65 and $-430 \mathrm{meV}$, respectively. The lines are drawn to determine the energetic width of the onset of these surface bands $((a, b)$ (C) R. Berndt, Kiel); (c) $\mathrm{d} I / \mathrm{d} V$ intensity as a function of position away from a step edge of $\mathrm{Cu}(111)$ measured at the voltages $\left(E-E_{\mathrm{F}}\right)$, as indicated (points); the lines are fits assuming standing electron waves with a phase coherence length $L_{\Phi}$ as marked; (d) resulting phase coherence time as a function of energy for $\mathrm{Ag}(111)$ and $\mathrm{Cu}(111)$. Inset shows the same data on a double-logarithmic scale, evidencing the $E^{-2}$ dependence (line) $((\mathrm{c}, \mathrm{d}) \odot \mathrm{H}$. Brune, Lausanne)

leads to a decay of the standing wave away from the step edge. The dotted fit line using $L_{\Phi}=\infty$ indicates this effect and, more importantly, shows a discrepancy from the measured curve. Only including a finite coherence length of $6.2 \mathrm{~nm}$ results in good agreement, which in turn determines $L_{\Phi}$ and thus $\tau_{\Phi}$, as displayed in Fig. 24.4c. The determined $1 / E^{2}$ dependence of $\tau_{\Phi}$ points to a dominating influence of electronelectron interactions at higher energies in the surface band.

\section{Landau and Spin Levels}

Moreover, the increased energy resolution at LT allows the resolution of electronic states that are not resolvable at room temperature (RT); for example, Landau and spin quantization appearing in a magnetic field $B$ have been probed on InAs(110) [24.11,71]. The corresponding quantization energies are given by $E_{\mathrm{Landau}}=$ $\hbar e B / m_{\text {eff }}$ and $E_{\text {spin }}=g \mu B$. Thus, InAs is a good choice, since it exhibits a low effective mass $m_{\text {eff }} / m_{\mathrm{e}}=0.023$ and a high $g$-factor of 14 in the bulk conduction band. The values in metals are $m_{\mathrm{eff}} / m_{\mathrm{e}} \approx 1$ and $g \approx 2$, resulting in energy splittings of only 1.25 and $1.20 \mathrm{meV}$ at $B=10 \mathrm{~T}$. This is obviously lower than the typical lifetime broadenings discussed in the previous section and also close to $\Delta E=1.1 \mathrm{meV}$ achievable at $T=$ $4 \mathrm{~K}$.

Fortunately, the electron density in doped semiconductors is much lower, and thus the lifetime increases significantly. Figure 24.5a shows a set of spectroscopy curves obtained on $\operatorname{InAs}(110)$ in different magnetic fields [24.11]. Above $E_{\mathrm{F}}$, oscillations with increasing intensity and energy distance are observed. They show the separation expected from Landau quantization. In turn, they can be used to deduce $m_{\text {eff }}$ from the peak separation (Fig. 24.5b). An increase of $m_{\mathrm{eff}}$ with increasing $E$ has been found, as expected from theory. Also, at high fields, spin quantization is observed (Fig. 24.5c). It is larger than expected from the bare $g$-factor due to contributions from exchange enhancement [24.72]. 
a) $\mathrm{d} / / \mathrm{d} V$ (arb. units)

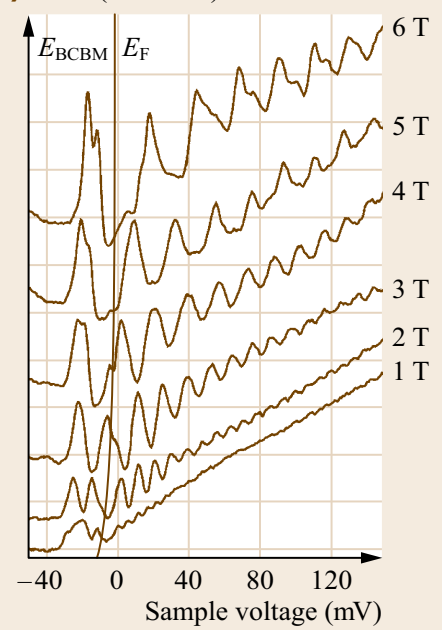

b) $m_{\text {eff }} / m_{\mathrm{e}}$

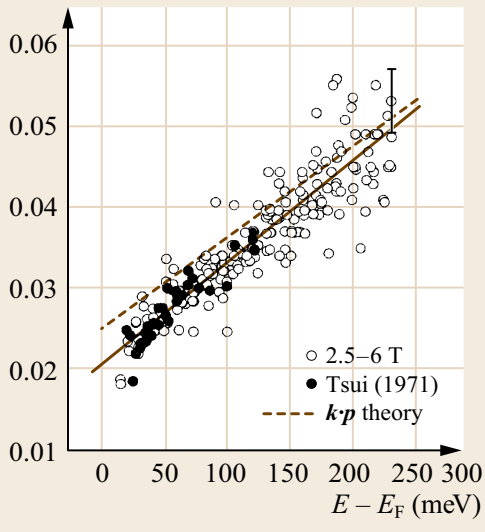

c) $\mathrm{d} I / \mathrm{d} V$ (arb. units)

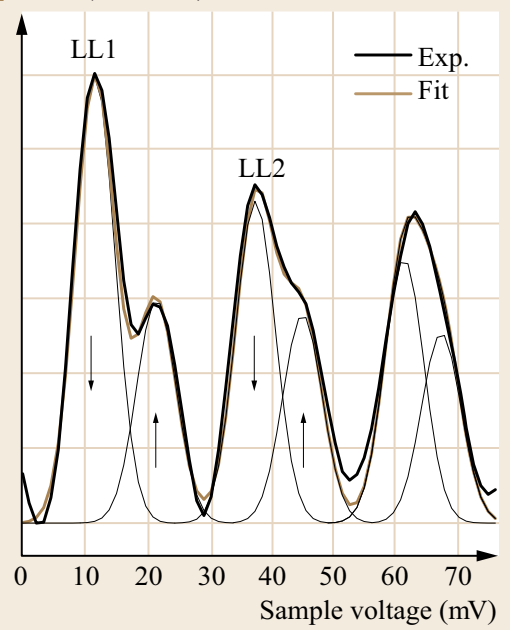

Fig. 24.5 (a) $\mathrm{d} I / \mathrm{d} V$ curves of $n$-InAs(110) at different magnetic fields, as indicated; $E_{\mathrm{BCBM}}$ marks the bulk conduction band minimum; oscillations above $E_{\mathrm{BCBM}}$ are caused by Landau quantization; the double peaks at $B=6 \mathrm{~T}$ are caused by spin quantization. (b) Effective-mass data deduced from the distance of adjacent Landau peaks $\Delta E$ according to $\Delta E=h e B / m_{\mathrm{eff}}$ (open symbols); filled symbols are data from planar tunnel junctions (Tsui), the solid line is a meansquare fit of the data and the dashed line is the expected effective mass of InAs according to $\boldsymbol{k} \cdot \boldsymbol{p}$ theory. (c) Magnification of a $\mathrm{d} I / \mathrm{d} V$ curve at $B=6 \mathrm{~T}$, exhibiting spin splitting; the Gaussian curves marked by arrows are the fitted spin levels

\section{Atomic Energy Levels}

Another opportunity at LT is to study electronic states and resonances of single adatoms. A complicated resonance is the Kondo resonance described below. A simpler resonance is a surface state bound at the adatom potential. It appears as a spatially localized peak below the onset of the extended surface state (Fig. 24.4a) [24.73, 74]. A similar resonance caused by a mixing of bulk states of the $\mathrm{NiAl}(110)$ substrate with atomic Au levels has been used to detect exchange splitting in Au dimers as a function of interatomic distance [24.75]. Single magnetic adatoms on the same surface also exhibit a double-peak resonance, but here due to the influence of spin-split d-levels of the adsorbate [24.76]. Atomic and molecular states decoupled from the substrate have finally been observed, when the atoms or molecules are deposited on an insulating thin film $[24.22,57]$.

\section{Vibrational Levels}

Inelastic tunneling processes contribute to the tunneling current. The coupling of electronic states to vibrational levels is one source of inelastic tunneling [24.33]. It provides additional channels contributing to $\mathrm{d} I / \mathrm{d} V(V)$ with final states at energies different from $V$. The final energy is simply shifted by the energy of the vibrational level. If only discrete vibrational energy levels couple to a smooth electronic DOS, one expects a peak in $\mathrm{d}^{2} I / \mathrm{d} V^{2}$ at the vibrational energy. This situation ap- pears for molecules on noble-metal surfaces. As usual, the isotope effect can be used to verify the vibrational origin of the peak. First indications of vibrational levels have been found for $\mathrm{H}_{2} \mathrm{O}$ and $\mathrm{D}_{2} \mathrm{O}$ on $\mathrm{TiO}_{2}$ [24.77], and completely convincing work has been performed for $\mathrm{C}_{2} \mathrm{H}_{2}$ and $\mathrm{C}_{2} \mathrm{D}_{2}$ on $\mathrm{Cu}(001)$ [24.33] (Fig. 24.6a). The technique has been used to identify individual molecules on the surface by their characteristic vibrational levels [24.60]. Moreover, the orientation of complexes with respect to the surface can be determined to a certain extent, since the vibrational excitation depends on the position of the tunneling current within the molecule. Finally, the excitation of certain molecular levels can induce such corresponding motions as hopping [24.50], rotation [24.52] (Fig. 24.6b-e), or desorption [24.49] leading to additional possibilities for manipulation on the atomic scale.

In turn, the manipulation efficiency as a function of applied voltage can be used to identify vibrational energies within the molecule, even if they are not detectable directly by $\mathrm{d}^{2} I / \mathrm{d} V^{2}$ spectroscopy [24.78]. Multiple vibronic excitations are found by positioning the molecule on an insulating film, leading to the observation of equidistant peaks in $\mathrm{d}^{2} I / \mathrm{d} V^{2}(V)$ [24.79].

\section{Kondo Resonance}

A rather intricate interaction effect is the Kondo effect. It results from a second-order scattering process be- 

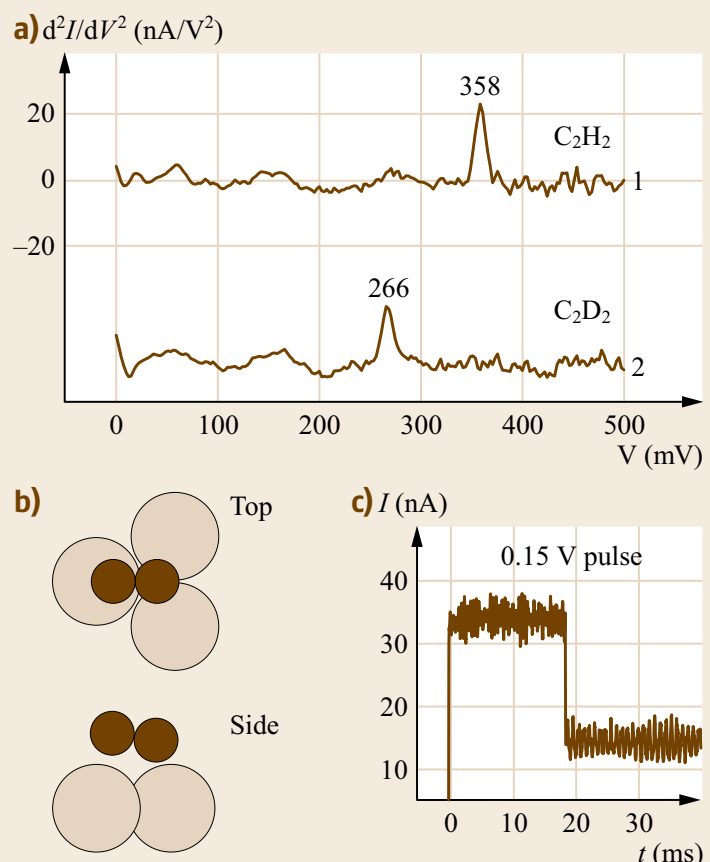

c) $I(\mathrm{nA})$
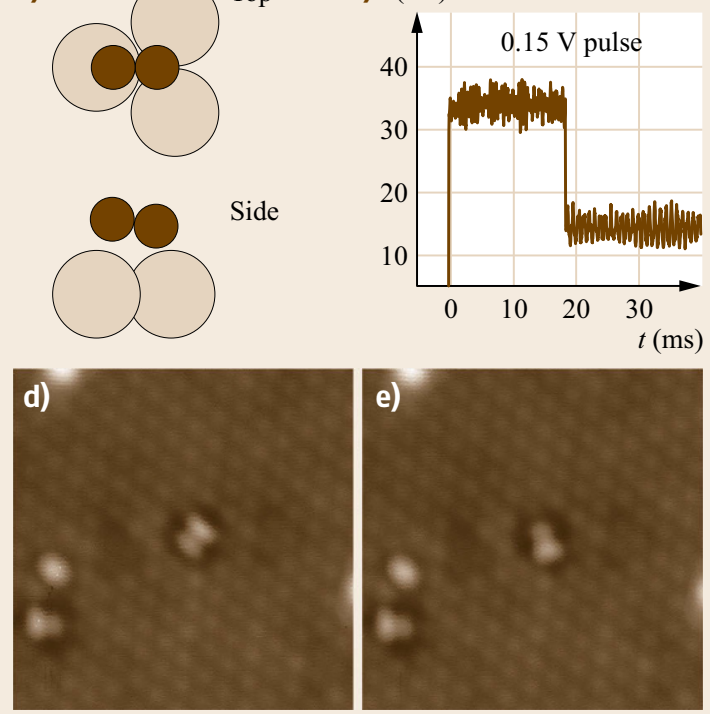

Fig. 24.6 (a) $\mathrm{d}^{2} I / \mathrm{d} V^{2}$ curves taken above a $\mathrm{C}_{2} \mathrm{H}_{2}$ and a $\mathrm{C}_{2} \mathrm{D}_{2}$ molecule on $\mathrm{Cu}(100)$; the peaks correspond to the $\mathrm{C}-\mathrm{H}$ or $\mathrm{C}-\mathrm{D}$ stretch-mode energy of the molecule, respectively. (b) Sketch of $\mathrm{O}_{2}$ molecule on $\mathrm{Pt}(111)$. (c) Tunneling current above an $\mathrm{O}_{2}$ molecule on $\mathrm{Pt}(111)$ during a voltage pulse of $0.15 \mathrm{~V}$; the jump in current indicates rotation of the molecule. $(d, e)$ STM images of an $\mathrm{O}_{2}$ molecule on $\operatorname{Pt}(111)(V=0.05 \mathrm{~V})$, prior to and after rotation induced by a voltage pulse to $0.15 \mathrm{~V}((\mathrm{a}-\mathrm{e})$ (C) $\mathrm{W}$. Ho, Irvine)

tween itinerate states and a localized state [24.80]. The two states exchange some degree of freedom back and forth, leading to a divergence of the scattering probability at the Fermi level of the itinerate state. Because of the divergence, the effect strongly modifies sample properties. For example, it leads to an unexpected increase in resistance with decreasing temperature for metals containing magnetic impurities [24.5]. Here, the exchanged degree of freedom is the spin. A spectroscopic signature of the Kondo effect is a narrow peak in the DOS at the Fermi level, continuously disappearing above a characteristic temperature (the Kondo temperature). STS provides the opportunity to study this effect on the local scale [24.81,82].

Figure 24.7a-d shows an example of Co clusters deposited on a carbon nanotube [24.83]. While only a small dip at the Fermi level, probably caused by curvature influences on the $\pi$-orbitals, is observed without Co (Fig. 24.7b) [24.84], a strong peak is found around a Co cluster deposited on top of the tube (Co cluster is marked in Fig. 24.7a). The peak is slightly shifted with respect to $V=0 \mathrm{mV}$ due to the so-called Fano resonance [24.85] which results from interference of the tunneling processes into the localized Co level and the itinerant nanotube levels. The resonance disappears within several nanometers of the cluster, as shown in Fig. 24.7d.

The Kondo effect has also been detected for different magnetic atoms deposited on noble-metal surfaces [24.81,82]. There, it disappears at about $1 \mathrm{~nm}$ from the magnetic impurity, and the effect of the Fano resonance is more pronounced, contributing to dips in $\mathrm{d} I / \mathrm{d} V(V)$ curves instead of peaks. Detailed investigations show that the d-level occupation of the adsorbate [24.86] as well as the surface charge density $[24.87,88]$ matter for the Kondo temperature. Exchange interaction between adsorbates tunable by their mutual distance can be used to tune the Kondo temperature [24.89] or even to destroy the Kondo resonance completely [24.90]. Meanwhile, magnetic molecules have also been shown to exhibit Kondo resonances. This increases the tunability of the Kondo effect, e.g., by the selection of adequate ligands surrounding the localized spins [24.91,92], by distant association of other molecules [24.93], or by conformational changes within the molecule [24.94].

A fascinating experiment has been performed by Manoharan et al. [24.95], who used manipulation to form an elliptic cage for the surface states of $\mathrm{Cu}(111)$ (Fig. 24.7e, bottom part). This cage was constructed to have a quantized level at $E_{\mathrm{F}}$. Then, a Co atom was placed in one focus of the elliptic cage, producing a Kondo resonance. Surprisingly, the same resonance reappeared in the opposite focus, but not away from the focus (Fig. 24.7e, top part). This shows amazingly that complex local effects such as the Kondo resonance can be wave-guided to remote points.

\subsubsection{Imaging Electronic Wave Functions}

\section{Bloch Waves}

Since STS measures the sum of squared wavefunctions (24.1), it is an obvious task to measure the local appearance of the simplest wavefunctions in solids, 


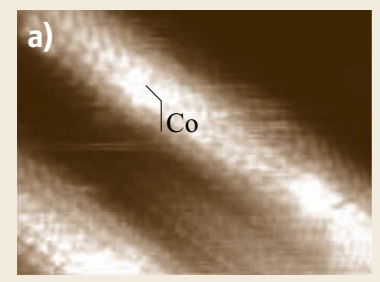

b) $\mathrm{d} I / \mathrm{d} V$ (arb. units)

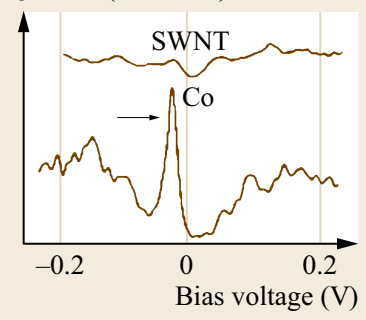

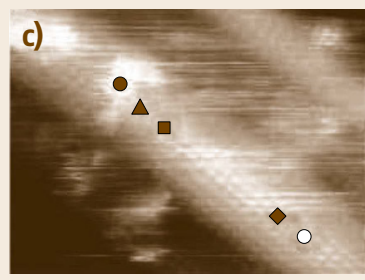

d) $\mathrm{d} / / \mathrm{d} V$ (arb. units)

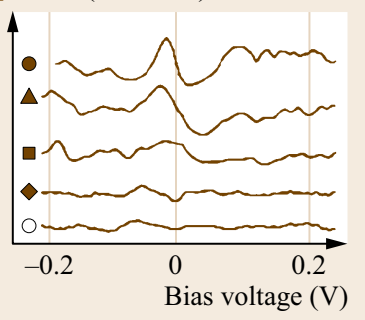

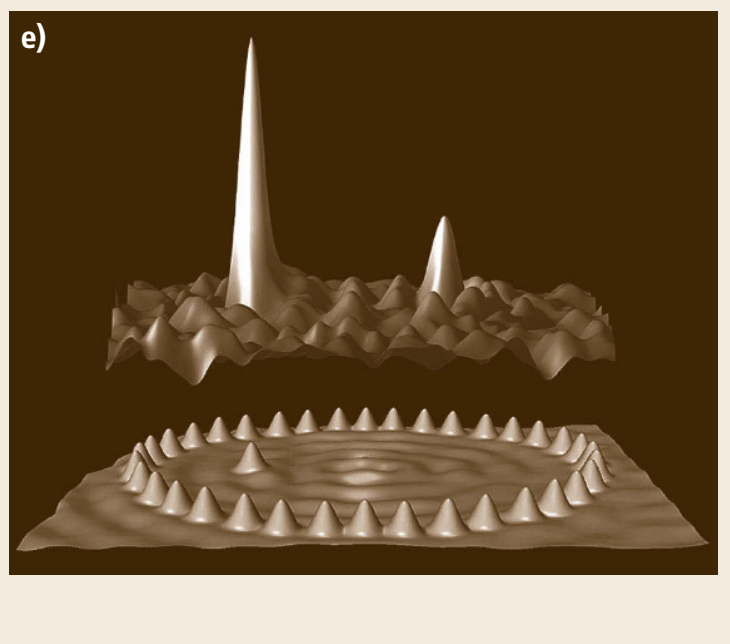

Fig. 24.7 (a) STM image of a Co cluster on a single-wall carbon nanotube (SWNT). (b) $\mathrm{d} I / \mathrm{d} V$ curves taken directly above the Co cluster (Co) and far away from the Co cluster (SWNT); the arrow marks the Kondo peak. (c) STM image of another Co cluster on a SWNT with symbols marking the positions where the $\mathrm{d} I / \mathrm{d} V$ curves displayed in (d) are taken. (d) $\mathrm{d} I / \mathrm{d} V$ curves taken at the positions marked in (c) ((a-d) (C) C. Lieber, Cambridge). (e) Lower part: STM image of a quantum corral of elliptic shape made from $\mathrm{Co}$ atoms on $\mathrm{Cu}(111)$; one $\mathrm{Co}$ atom is placed at one of the foci of the ellipse. Upper part: map of the strength of the Kondo signal in the corral; note that there is also a Kondo signal at the focus that is not covered by a Co atom ((e) (C) D. Eigler, Almaden)

namely Bloch waves. The atomically periodic part of the Bloch wave is always measured if atomic resolution is achieved (inset of Fig. 24.9a). However, the long-range wavy part requires the presence of scatterers. The electron wave impinges on the scatterer and is reflected, leading to self-interference. In other words, the phase of the Bloch wave becomes fixed by the scatterer.

Such self-interference patterns were first found on graphite (0001) [24.96] and later on noble-metal surfaces, where adsorbates or step edges scatter the surface states (Fig. 24.8a) [24.30]. Fourier transforms of the real-space images reveal the $k$-space distribution of the corresponding states [24.97], which may include additional contributions besides the surface state [24.98]. Using particular geometries such as so-called quantum corrals, the Bloch waves can be confined (Fig. 24.8b). Depending on the geometry of the corral, the resulting state looks rather complex, but it can usually be reproduced by simple calculations involving single-particle states only [24.99].

Meanwhile, Bloch waves in semiconductors scattered at charged dopants (Fig. 24.8c,d) [24.100], Bloch states confined in semiconducting or organic quantum dots (Fig. 24.8e-g) [24.101-103] and quantum wells [24.104], as well as Bloch waves confined in short-cut carbon nanotubes (Fig. 24.8h,i) [24.105, 106] have been visualized. In special nanostructures, it was even possible to extract the phase of the wavefunction by using the mathematically known transformation matrices of so-called isospectral structures, i.e., geometrically different structures exhibiting exactly the same spatially averaged density of states. The resulting wavefunctions $\Psi(x)$ are shown in Fig. 24.8j [24.107].

More localized structures, where a Bloch wave description is not appropriate, have been imaged, too. Examples are the highest occupied molecular orbital (HOMO) and lowest unoccupied molecular orbital (LUMO) of pentacene molecules deposited on $\mathrm{NaCl} / \mathrm{Cu}(100)$ (Fig. 24.8k,1) [24.57], the different molecular states of $\mathrm{C}_{60}$ on $\mathrm{Ag}(110)$ (Fig. 24.8mo) [24.108], the anisotropic states of $\mathrm{Mn}$ acceptors in a semiconducting host $[24.109,110]$, and the hybridized states developing within short monoatomic $\mathrm{Au}$ chains, which result in particular states at the end of the chains [24.111, 112]. Using pairs of remote Mn acceptors, even symmetric and antisymmetric pair wavefunctions have been imaged in real space [24.113].

The central requirements for a detailed imaging of wavefunctions are LT for an appropriate energetic distinction of an individual state, adequate decoupling of the state from the substrate in order to decrease lifetimeinduced broadening effects, and, partly, the selection of a system with an increased Bohr radius in order to increase the spatial extension of details above the lat- 
Fig. 24.8a-d (a) Low-voltage STM image of $\mathrm{Cu}(111)$ including two defect atoms; the waves are electronic Bloch waves scattered at the defects; (b) low-voltage STM image of a rectangular quantum corral made from single atoms on $\mathrm{Cu}(111)$; the pattern inside the corral is the confined state of the corral close to $E_{\mathrm{F}}$; (@ D. Eigler, Almaden $(\mathrm{a}, \mathrm{b})$ ); (c) STM image of GaAs(110) around a Si donor, $V=$ $-2.5 \mathrm{~V}$; the line scan along $\mathrm{A}$, shown in (d), exhibits an additional oscillation around the donor caused by a standing Bloch wave; the grid-like pattern corresponds to the atomic corrugation of the Bloch wave (@ H. van Kempen, Nijmegen $(c, d)) ;(e-g) d I / d V$ images of a self-assembled InAs quantum dot deposited on $\operatorname{GaAs}(100)$ and measured at different $V((\mathrm{e}) 1.05 \mathrm{~V}$, (f) $1.39 \mathrm{~V}$, (g) $1.60 \mathrm{~V})$. The images show the squared wavefunctions confined within the quantum dot, which exhibit zero, one, and two nodal lines with increasing energy. (h) STM image of a short-cut carbon nanotube; (i) greyscale plot of the $\mathrm{d} I / \mathrm{d} V$ intensity inside the short-cut nanotube as a function of position $(x$-axis) and tunneling voltage ( $y$-axis); four wavy patterns of different wavelength are visible in the voltage range from -0.1 to $0.15 \mathrm{~V}$ (@ C. Dekker, Delft $(\mathrm{h}, \mathrm{i})$ ); (j) two reconstructed wavefunctions confined in so-called isospectral corrals made of $\mathrm{CO}$ molecules on $\mathrm{Cu}(111)$. Note that $\Psi(x)$ instead of $|\Psi(x)|^{2}$ is displayed, exhibiting positive and negative values. This is possible since the transplantation matrix transforming one isospectral wavefunction into another is known (๑) H. Manoharan, Stanford (j)); (k,I) STM images of a pentacene molecule deposited on $\mathrm{NaCl} / \mathrm{Cu}(100)$ and measured with a pentacene molecule at the apex of the tip at $V=-2.5 \mathrm{~V}$ $((\mathbf{k})$ HOMO $=$ highest occupied molecular orbital) and $V=2.5 \mathrm{~V}$ ((I) LUMO = lowest unoccupied molecular orbital) (@) J. Repp, Regensburg (k,l)); (m) STM image of a $\mathrm{C}_{60}$ molecule deposited on $\operatorname{Ag}(100), V=2.0 \mathrm{~V}$; $(\mathrm{n}, 0) \mathrm{d} I / \mathrm{d} V$ images of the same molecule at $V=0.4 \mathrm{~V}(\mathrm{n})$, $1.6 \mathrm{~V}(\mathrm{n})((\mathrm{m}-\mathrm{o}) \odot \mathrm{M}$. Crommie, Berkeley $)$

eral resolution of STM, thereby improving, e.g., the visibility of bonding and antibonding pair states within a dimer [24.113].

\section{Wavefunctions in Disordered Systems}

More complex wavefunctions result from interactions. A nice playground to study such interactions is doped semiconductors. The reduced electron density with respect to metals increases the importance of electron interactions with potential disorder and other electrons. Applying a magnetic field quenches the kinetic energy, thus enhancing the importance of interactions. A dramatic effect can be observed on InAs(110), where threedimensional (3-D) bulk states are measured. While the usual scattering states around individual dopants are observed at $B=0$ T (Fig. 24.9a) [24.114], stripe structures
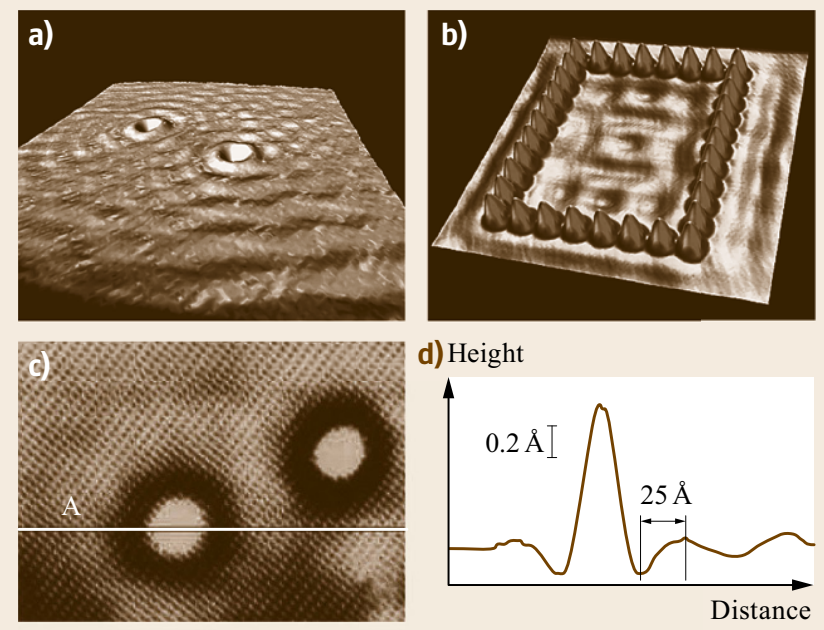

\section{d) Height}
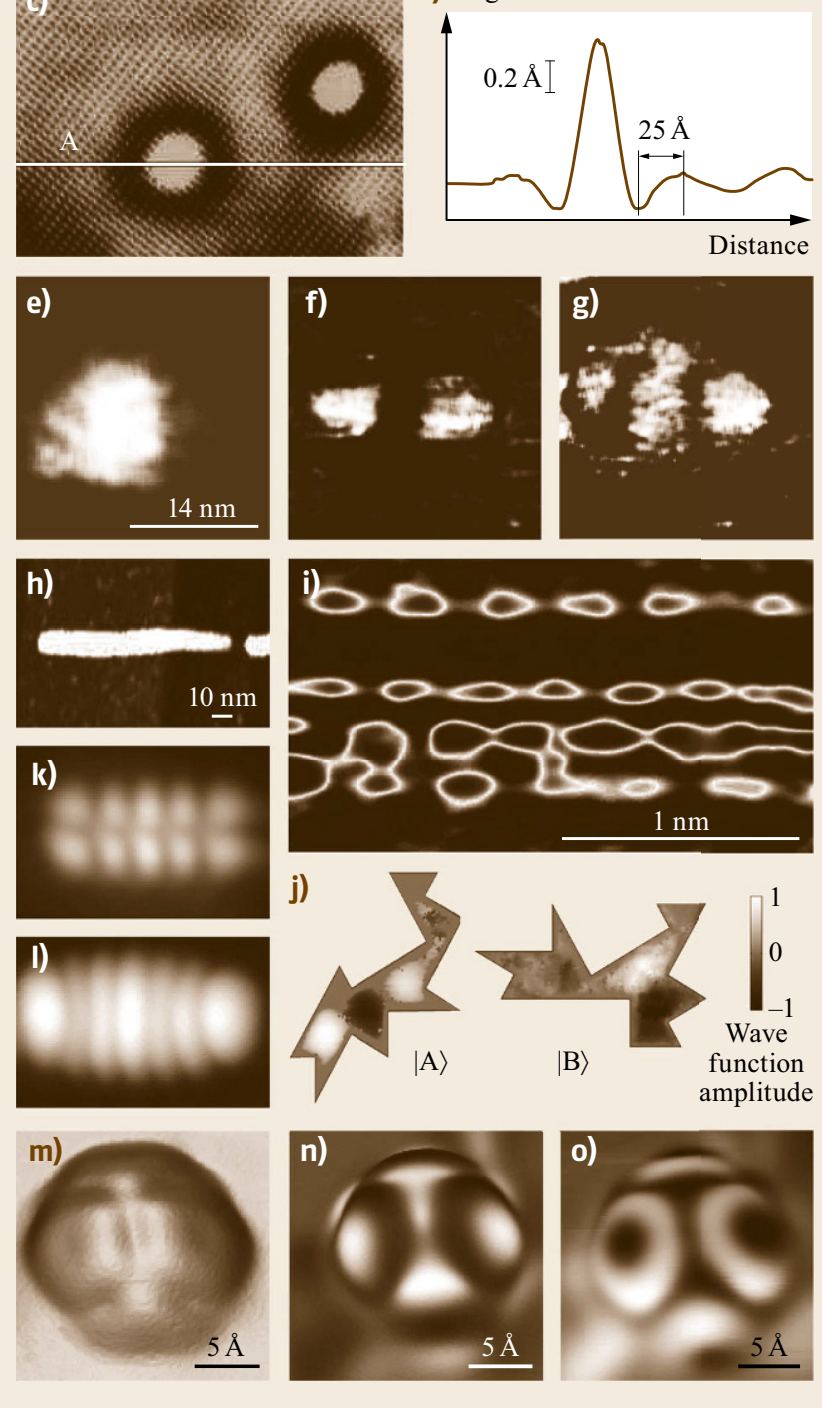

are found at high magnetic field (Fig. 24.9b) [24.115]. They run along equipotential lines of the disorder potential. This can be understood by recalling that the electron tries to move in a cyclotron circle, which becomes a cycloid path along an equipotential line within an inhomogeneous electrostatic potential [24.116]. 

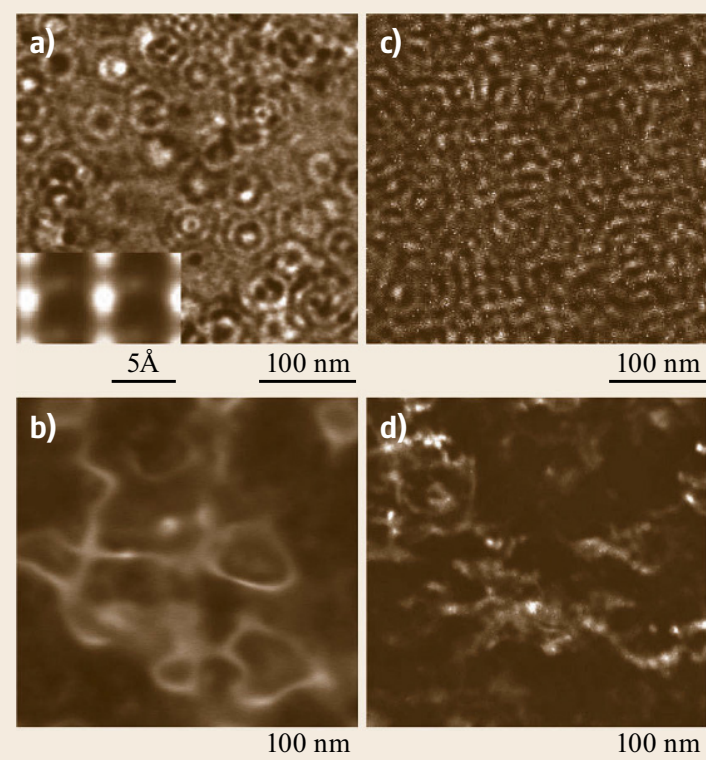

Fig. 24.9 (a) $\mathrm{d} I / \mathrm{d} V$ image of $\operatorname{InAs}(110)$ at $V=50 \mathrm{mV}$, $B=0 \mathrm{~T}$; circular wave patterns corresponding to standing Bloch waves around each sulphur donor are visible; inset shows a magnification revealing the atomically periodic part of the Bloch wave. (b) Same as (a), but at $B=6 \mathrm{~T}$; the stripe structures are drift states. (c) $\mathrm{d} I / \mathrm{d} V$ image of a 2$D$ electron system on $\operatorname{InAs}(110)$ induced by the deposition of Fe, $B=0 \mathrm{~T}$. (d) Same as (c) but at $B=6 \mathrm{~T}$; note that the contrast in (a) is increased by a factor of ten with respect to $(b-d)$

The same effect has been found in two-dimensional (2-D) electron systems (2-DES) of InAs at the same large $B$-field (Fig. 24.9d) [24.117]. However, the scattering states at $B=0 \mathrm{~T}$ are much more complex in 2-D (Fig. 24.9c) [24.118]. The reason is the tendency of a 2-DES to exhibit closed scattering paths [24.119]. Consequently, the self-interference does not result from scattering at individual scatterers, but from complicated self-interference paths involving many scatterers. Nevertheless, the wavefunction pattern can be reproduced by including these effects within the calculations.

Reducing the dimensionality to one dimension (1-D) leads again to complicated self-interference patterns due to the interaction of the electrons with several impurities [24.120,121]. For InAs, they can be reproduced by single-particle calculations. However, experiments imaging self-interference patterns close to the end of a C-nanotube are interpreted as indications of spin charge separation, a genuine property of 1-D electrons not feasible within the single-particle description [24.122].
Charge Density Waves, Jahn-Teller Distortion Another interaction modifying the LDOS is the electron-phonon interaction. Phonons scatter electrons between different Fermi points. If the wavevectors connecting Fermi points exhibit a preferential orientation, a so-called Peierls instability occurs [24.123]. The corresponding phonon energy goes to zero, the atoms are slightly displaced with the periodicity of the corresponding wavevector, and a charge density wave (CDW) with the same periodicity appears. Essentially, the CDW increases the overlap of the electronic states with the phonon by phase fixing with respect to the atomic lattice. The Peierls transition naturally occurs in one-dimensional (1-D) systems, where only two Fermi points are present and hence preferential orientation is excessive. It can also occur in 2-D systems if large parts of the Fermi line run in parallel.

STS studies of CDWs are numerous [24.124, 125]. Examples of a 1-D CDW on a quasi-1-D bulk material and of a 2-D CDW are shown in Fig. 24.10a-d and Fig. 24.10e-h, respectively [24.126, 127]. In contrast to usual scattering states, where LDOS corrugations are only found close to the scatterer, the corrugations of CDWs are continuous across the surface. Heating the substrate toward the transition temperature leads to a melting of the CDW lattice, as shown in Fig. 24.10f-h.

CDWs have also been found on monolayers of adsorbates such as a monolayer of $\mathrm{Pb}$ on $\mathrm{Ge}(111)$ [24.128]. These authors performed a nice temperature-dependent study revealing that the CDW is nucleated by scattering states around defects, as one might expect [24.129]. Some of the transitions have been interpreted as more complex Mott-Hubbard transitions caused primarily by electron-electron interactions [24.130]. One-dimensional systems have also been prepared on surfaces showing Peierls transitions [24.131, 132]. Finally, the energy gap occurring at the transition has been studied by measuring $\mathrm{d} I / \mathrm{d} V(V)$ curves [24.133].

A more local crystallographic distortion due to electron-lattice interactions is the Jahn-Teller effect. Here, symmetry breaking by elastic deformation can lead to the lifting of degeneracies close to the Fermi level. This results in an energy gain due to the lowering of the energy of the occupied levels. By tuning the Fermi level of an adsorbate layer to a degeneracy via doping, such a Jahn-Teller deformation has been induced on a surface and visualized by STM [24.134].

\section{Superconductors}

An intriguing effect resulting from electron-phonon interaction is superconductivity. Here, the attractive part of the electron-phonon interaction leads to the coupling of electronic states with opposite wave vector 

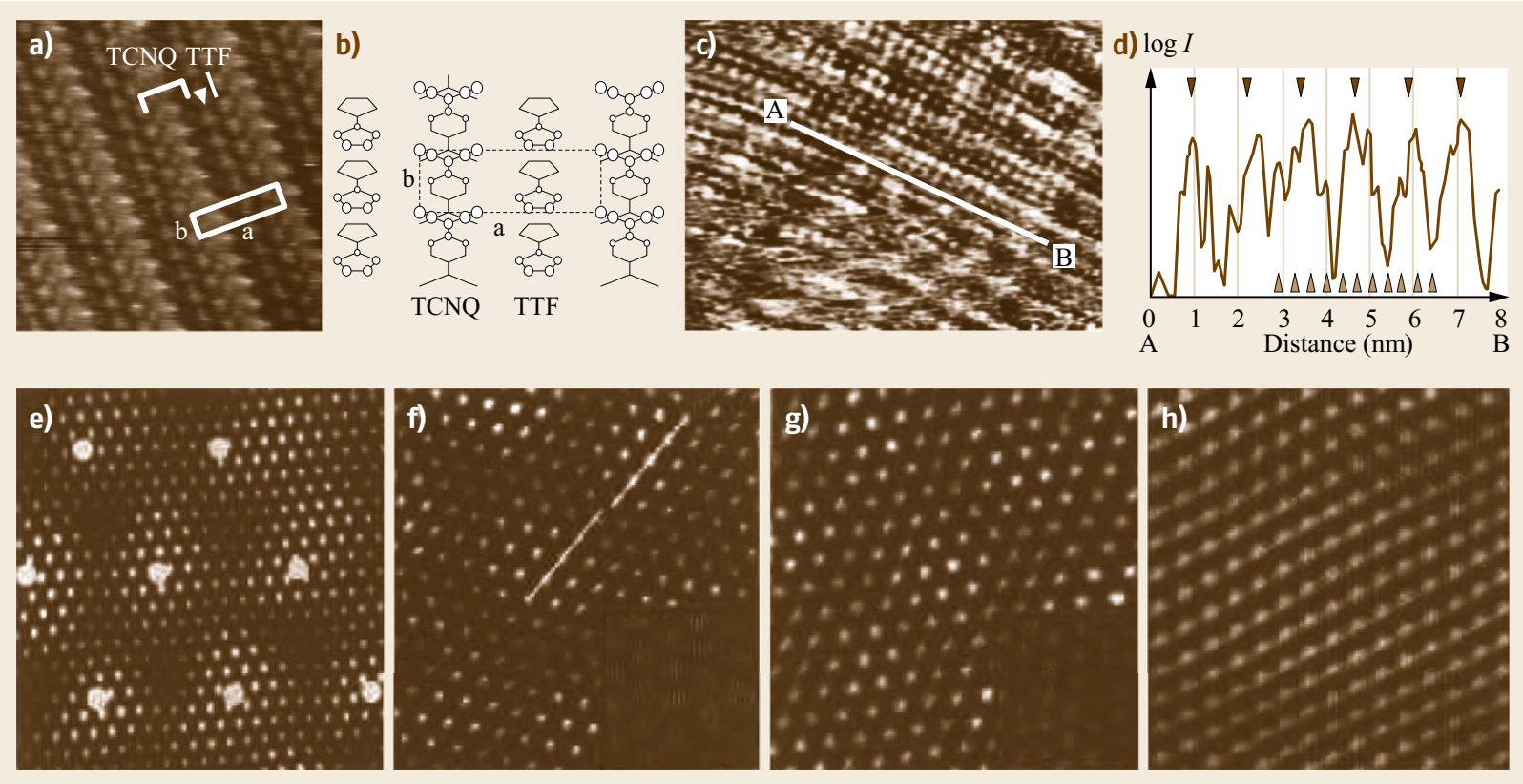

Fig. 24.10 (a) STM image of the $a b$-plane of the organic quasi-1-D conductor tetrathiafulvalene tetracyanoquinodimethane (TTFTCNQ), $T=300 \mathrm{~K}$; while the TCNQ chains are conducting, the TTF chains are insulating. (b) Stick-and-ball model of the $a b$-plane of TTF-TCNQ. (c) STM image taken at $T=61 \mathrm{~K}$; the additional modulation due to the Peierls transition is visible in the profile along line $\mathrm{AB}$ shown in (d); the brown triangles mark the atomic periodicity and the black triangles the expected CDW periodicity ( (a-d) (C) M. Kageshima, Kanagawa). (e-h) Low-voltage STM images of the two-dimensional CDW system 1 T-TaS 2 at $T=242 \mathrm{~K}(\mathrm{e}), 298 \mathrm{~K}(\mathrm{f}), 349 \mathrm{~K}(\mathrm{~g})$, and $357 \mathrm{~K}(\mathrm{~h})$. A long-range, hexagonal modulation is visible besides the atomic spots; its periodicity is highlighted by large white dots in (e); the additional modulation obviously weakens with increasing $T$, but is still apparent in (f) and (g), as evidenced in the lower-magnification images in the insets $(\mathbf{( e - h ) ~ ( ) ~ C . ~ L i e b e r , ~ C a m b r i d g e ) ~}$

and mostly opposite spin [24.135]. Since the resulting Cooper pairs are bosons, they can condense at LT, forming a coherent many-particle phase, which can carry current without resistance. Interestingly, defect scattering does not influence the condensate if the coupling along the Fermi surface is homogeneous (s-wave superconductor). The reason is that the symmetry of the scattering of the two components of a Cooper pair effectively leads to a scattering from one Cooper pair state to another without affecting the condensate. This is different if the scatterer is magnetic, since the different spin components of the pair are scattered differently, leading to an effective pair breaking, which is visible as a single-particle excitation within the superconducting gap. On a local scale, this effect was first demonstrated by putting $\mathrm{Mn}, \mathrm{Gd}$, and $\mathrm{Ag}$ atoms on a $\mathrm{Nb}(110)$ surface [24.136]. While the nonmagnetic $\mathrm{Ag}$ does not modify the gap shown in Fig. 24.11a, it is modified in an asymmetric fashion close to Mn or Gd adsorbates, as shown in Fig. 24.11b. The asymmetry of the additional intensity is caused by the breaking of the particle-hole symmetry due to the exchange interaction between the localized $\mathrm{Mn}$ state and the itinerate $\mathrm{Nb}$ states.
Another important local effect is caused by the relatively large coherence length of the condensate. At a material interface, the condensate wavefunction cannot stop abruptly, but overlaps into the surrounding material (proximity effect). Consequently, a superconducting gap can be measured in areas of nonsuperconducting material. Several studies have shown this effect on the local scale using metals and doped semiconductors as surrounding materials $[24.137,138]$.

While the classical type I superconductors are ideal diamagnets, the so-called type II superconductors can contain magnetic flux. The flux forms vortices, each containing one flux quantum. These vortices are accompanied by the disappearance of the superconducting gap and, therefore, can be probed by STS [24.139]. LDOS maps measured inside the gap lead to bright features in the area of the vortex core. Importantly, the length scale of these features is different from the length scale of the magnetic flux due to the difference between the London penetration depth and the electronic coherence length. Thus, STS probes a different property of the vortex than the usual magnetic imaging techniques (Sect. 24.4.5). Surprisingly, first measurements of the 


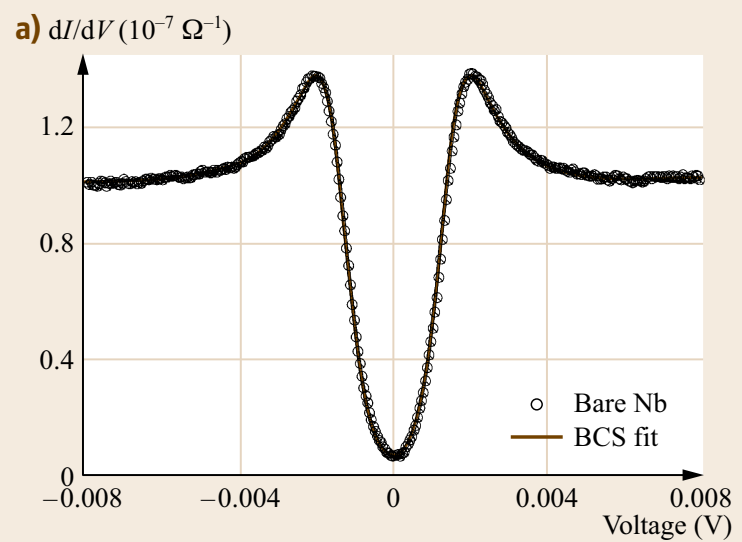

b) $\mathrm{d} / / \mathrm{d} V$ difference $\left(10^{-7} \Omega^{-1}\right)$
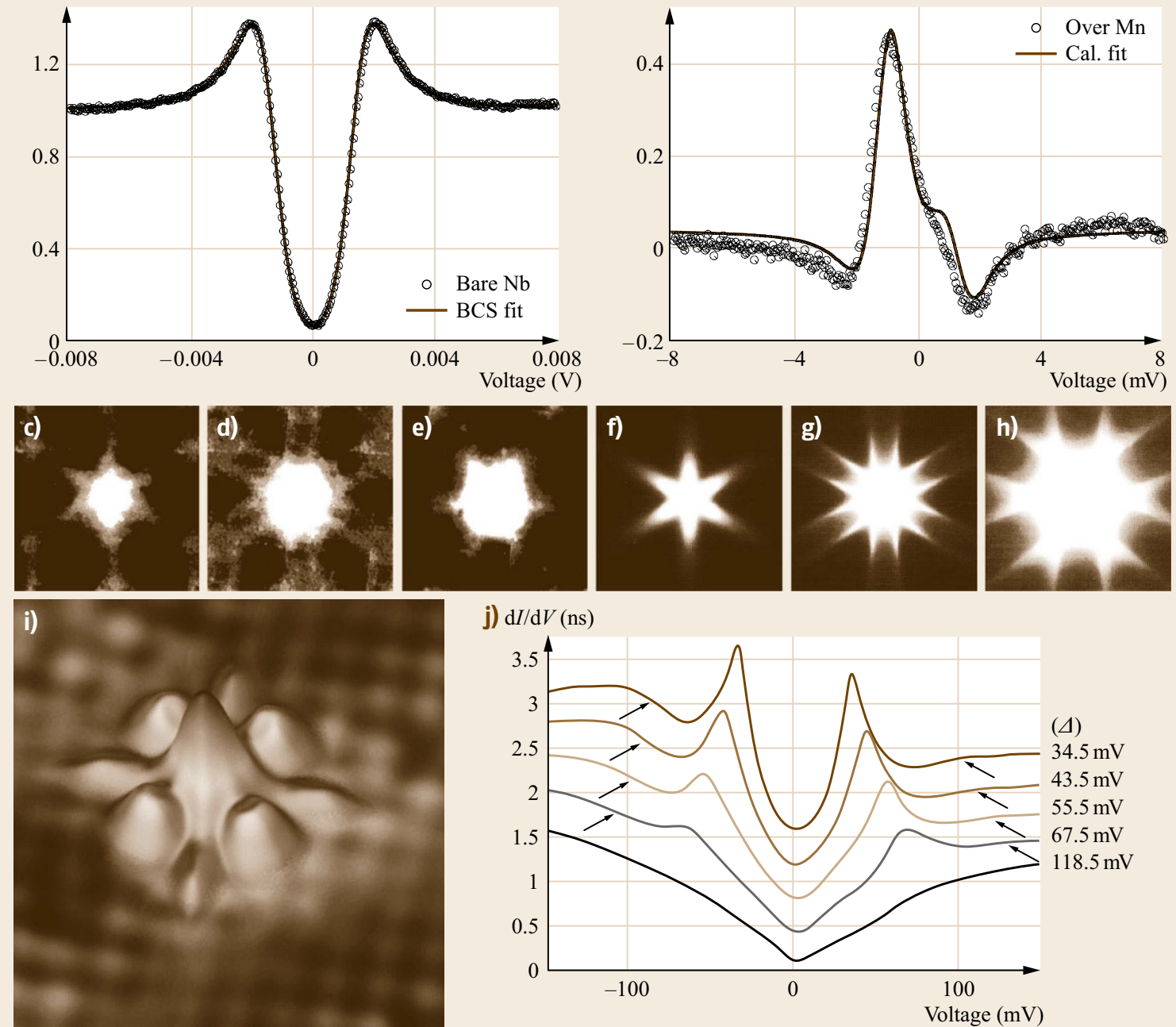

j) $\mathrm{d} / / \mathrm{d} V$ (ns)
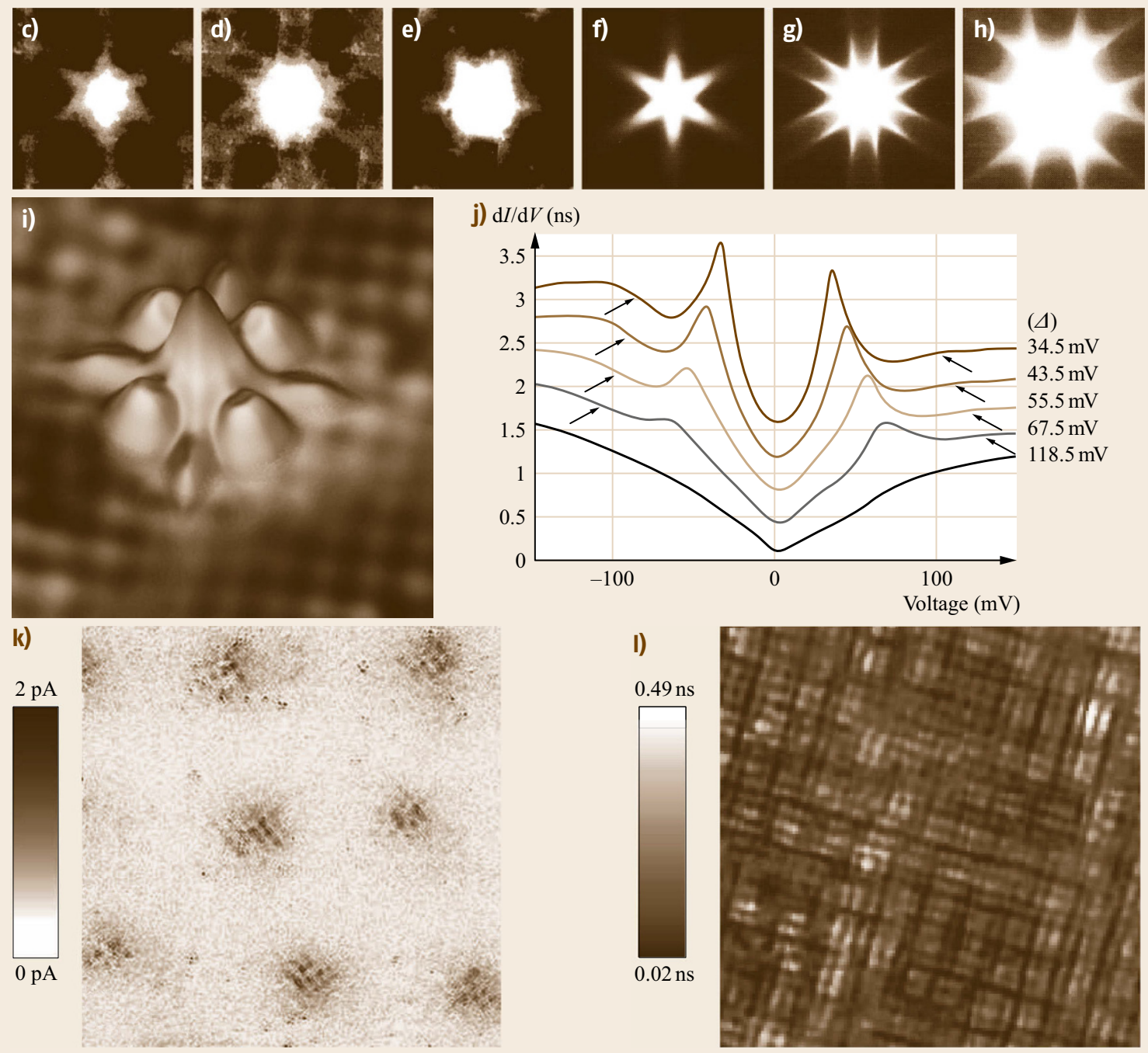

$100 \AA$ 
Fig. 24.11 (a) $\mathrm{d} I / \mathrm{d} V$ curve of $\mathrm{Nb}(110)$ at $T=3.8 \mathrm{~K}$ (symbols) in comparison with a BCS fit of the superconducting gap of $\mathrm{Nb}$ (line). (b) Difference between the $\mathrm{d} I / \mathrm{d} V$ curve taken directly above a $\mathrm{Mn}$ atom on $\mathrm{Nb}(110)$ and the $\mathrm{d} I / \mathrm{d} V$ curve taken above clean $\mathrm{Nb}(110)$ (symbols) in comparison with a fit using the Bogulubov-de Gennes equations (line) (@ D. Eigler, Almaden $(\mathrm{a}, \mathrm{b})$ ). (c-e) $\mathrm{d} I / \mathrm{d} V$ images of a vortex core in the type II superconductor $2 \mathrm{H}-\mathrm{NbSe}_{2}$ at $0 \mathrm{mV}$ (c), $0.24 \mathrm{mV}$ (d), and $0.48 \mathrm{mV}$ (e) ((c-e) (C) H.F. Hess). (f-h) Corresponding calculated LDOS images within the Eilenberger framework ((f-h) @ $\mathrm{K}$. Machida, Okayama). (i) Overlap of an STM image at $V=-100 \mathrm{mV}$ (background 2-D image) and a $I I / \mathrm{d} V$ image at $V=0 \mathrm{mV}$ (overlapped 3-D image) of optimally doped $\mathrm{Bi}_{2} \mathrm{Sr}_{2} \mathrm{CaCu}_{2} \mathrm{O}_{8+\delta}$ containing $0.6 \% \mathrm{Zn}$ impurities. The STM image shows the atomic structure of the cleavage plane, while the $\mathrm{d} I / \mathrm{d} V$ image shows a bound state within the superconducting gap, which is located around a single $\mathrm{Zn}$ impurity. The fourfold symmetry of the bound state reflects the d-like symmetry of the superconducting pairing function; (j) $\mathrm{d} I / \mathrm{d} V$ spectra of $\mathrm{Bi}_{2} \mathrm{Sr}_{2} \mathrm{CaCu}_{2} \mathrm{O}_{8+\delta}$ measured at different positions of the surface at $T=4.2 \mathrm{~K}$; the phonon peaks are marked by arrows, and the determined local gap size $\Delta$ is indicated; note that the strength of the phonon peak increases with the strength of the coherence peaks surrounding the gap; (k) LDOS in the vortex core of slightly overdoped $\mathrm{Bi}_{2} \mathrm{Sr}_{2} \mathrm{CaCu}_{2} \mathrm{O}_{8+\delta}, B=$ $5 \mathrm{~T}$; the $\mathrm{d} I / \mathrm{d} V$ image taken at $B=5 \mathrm{~T}$ is integrated over $V=1-12 \mathrm{mV}$, and the corresponding $\mathrm{d} I / \mathrm{d} V$ image at $B=0 \mathrm{~T}$ is subtracted to highlight the LDOS induced by the magnetic field. The checkerboard pattern within the seven vortex cores exhibits a periodicity, which is fourfold with respect to the atomic lattice shown in (i) and is thus assumed to be a CDW; (I) STM image of cleaved $\mathrm{Ca}_{1.9} \mathrm{Na}_{0.1} \mathrm{CuO}_{2} \mathrm{Cl}_{2}$ at $T=0.1 \mathrm{~K}$, i.e., within the superconducting phase of the material; a checkerboard pattern with fourfold periodicity is visible on top of the atomic resolution (@ S. Davis, Cornell and S. Uchida, Tokyo (i-I)) <

vortices on $\mathrm{NbSe}_{2}$ revealed vortices shaped as a sixfold star [24.140] (Fig. 24.11c). With increasing voltage inside the gap, the orientation of the star rotates by $30^{\circ}$ (Fig. $24.11 \mathrm{~d}, \mathrm{e}$ ). The shape of these stars could finally be reproduced by theory, assuming an anisotropic pairing of electrons in the superconductor (Fig. 24.11fh) [24.141]. Additionally, bound states inside the vortex core, which result from confinement by the surrounding superconducting material, are found [24.140]. Further experiments investigated the arrangement of the vortex lattice, including transitions between hexagonal and quadratic lattices [24.142], the influence of pinning centers [24.143], and the vortex motion induced by current [24.144].
A central topic is still the understanding of hightemperature superconductors (HTSC). An almost accepted property of HTSC is their d-wave pairing symmetry, which is partly combined with other contributions [24.145]. The corresponding $\boldsymbol{k}$-dependent gap (where $\boldsymbol{k}$ is the reciprocal lattice vector) can be measured indirectly by STS using a Fourier transformation of the $\operatorname{LDOS}(x, y)$ determined at different energies [24.146]. This shows that LDOS modulations in HTSC are dominated by simple self-interference patterns of the Bloch-like quasiparticles [24.147]. However, scattering can also lead to pair breaking (in contrast to s-wave superconductors), since the Cooper-pair density vanishes in certain directions. Indeed, scattering states (bound states in the gap) around nonmagnetic $\mathrm{Zn}$ impurities have been observed in $\mathrm{Bi}_{2} \mathrm{Sr}_{2} \mathrm{CaCu}_{2} \mathrm{O}_{8}$. (BSCCO) (Fig. 24.11i,j) [24.148]. They reveal a dlike symmetry, but not the one expected from simple Cooper-pair scattering. Other effects such as magnetic polarization in the environment probably have to be taken into account [24.149]. An interesting topic is the importance of inhomogeneities in HTSC materials. Evidence for inhomogeneities has indeed been found in underdoped materials, where puddles of the superconducting phase identified by the coherence peaks around the gap are shown to be embedded in nonsuperconducting areas [24.36].

In addition, temperature-dependent measurements of the gap size development at each spatial position exhibit a percolation-type behavior above $T_{\mathrm{c}}$ [24.35]. This stresses the importance of inhomogeneities, but the observed percolation temperature being higher than $T_{\mathrm{c}}$ shows that $T_{\mathrm{c}}$ is not caused by percolation of superconducting puddles only. Conversely, it was found that for overdoped and optimally doped samples the gap develops continuously across $T_{\mathrm{c}}$, showing a universal relation between the local gap size $\Delta(T=0)$ (measured at low temperature) and the local critical temperature $T_{\mathrm{p}}$ (at which the gap completely disappears): $2 \Delta(T=0) /\left(k_{\mathrm{B}} T_{\mathrm{p}}\right) \approx 8$. The latter result is evidence that the so-called pseudogap phase is a phase with incoherent Cooper pairs. The results are less clear in the underdoped region, where probably two gaps complicate the analysis. Below $T_{\mathrm{c}}$, it turns out that the strength of the coherence peak is anticorrelated to the local oxygen acceptor density [24.147] and, in addition, correlated to the energy of an inelastic phonon excitation peak in $\mathrm{d} I / \mathrm{d} V$ spectra [24.37]. Figure $24.11 \mathrm{j}$ shows corresponding spectra taken at different positions, where the coherence peaks and the nearby phonon peaks marked by arrows are clearly visible. The phonon origin of the peak has been proven by the isotope effect, similar to Fig. 24.6a. The strong intensity of the phonon 
side-peak as well as the correlation of its strength with the coherence peak intensity points towards an important role of electron-phonon coupling for the pairing mechanism. However, since the gap size does not scale with the strength of the phonon peak [24.150], other contributions must be involved too.

Of course, vortices have also been investigated for HTSC [24.151]. Bound states are found, but at energies that are in disagreement with simple models, assuming a Bardeen-Cooper-Schrieffer (BCS)like d-wave superconductor $[24.152,153]$. Theory predicts, instead, that the bound states are magnetic-fieldinduced spin density waves, stressing the competition between antiferromagnetic order and superconductivity in HTSC materials [24.154]. Since the spin density wave is accompanied by a charge density wave of half wavelength, it can be probed by STS [24.155]. Indeed, a checkerboard pattern of the right periodicity has been found in and around vortex cores in BSCCO (Fig. 24.11k). Similar checkerboards, which do not show any $E(k)$ dispersion, have also been found in the underdoped pseudogap phase at temperatures higher than the superconducting transition temperature [24.156] or at dopant densities lower than the critical doping [24.157]. Depending on the sample, the patterns can be either homogeneous or inhomogeneous and exhibit slightly different periodicities. However, the fact that the pattern persists within the superconducting phase as shown in Fig. 24.111, at least for $\mathrm{Na}-\mathrm{CCOC}$, indicates that the corresponding phase can coexist with superconductivity. This raises the question of whether spin density waves are the central opponent to HTSC. Interestingly, a checkerboard pattern of similar periodicity, but without long-range order, is also found, if one displays the particle-hole asymmetry of $\mathrm{d} I / \mathrm{d} V(V)$ intensity in underdoped samples at low temperature [24.158]. Since the observed asymmetry is known to be caused by the lifting of the correlation gap with doping, the checkerboard pattern might be directly linked to the corresponding localized holes in the $\mathrm{CuO}$ planes appearing at low doping. Although a comprehensive model for HTSC materials is still lacking, STS contributes significantly to disentangling this puzzle.

Notice that all the measurements described above have probed the superconducting phase only indirectly by measuring the quasiparticle LDOS. The superconducting condensate itself could principally also be probed directly using Cooper-pair tunneling between a superconducting tip and a superconducting sample. A proof of principle of this detection scheme has indeed been given at low tunneling resistance $(R \approx$ $50 \mathrm{k} \Omega$ ) [24.159], but meaningful spatially resolved data are still lacking.

\subsubsection{Imaging Spin Polarization: Nanomagnetism}

Conventional STS couples to the LDOS, i. e., the charge distribution of the electronic states. Since electrons also have spin, it is desirable to also probe the spin distribution of the states. This can be achieved by spinpolarized STM (SP-STM) using a tunneling tip covered by a ferromagnetic material [24.160]. The coating acts as a spin filter or, more precisely, the tunneling current depends on the relative angle $\alpha_{\mathrm{ij}}$ between the spins of the tip and the sample according to $\cos \left(\alpha_{\mathrm{ij}}\right)$. Consequently, a particular tip is not sensitive to spin orientations of the sample perpendicular to the spin orientation of the tip. Different tips have to be prepared to detect different spin orientations. Moreover, the stray magnetic field of the tip can perturb the spin orientation of the sample. To avoid this, a technique using antiferromagnetic $\mathrm{Cr}$ as a tip coating material has been developed [24.161]. This avoids stray fields, but still provides a preferential spin orientation of the few atoms at the tip apex that dominate the tunneling current. Depending on the thickness of the $\mathrm{Cr}$ coating, spin orientations perpendicular or parallel to the sample surface, implying corresponding sensitivities to the spin directions of the sample, are achieved.

SP-STM has been used to image the evolution of magnetic domains with increasing $B$ field (Fig. 24.12ad) [24.162], the antiferromagnetic order of a Mn monolayer on W(110) [24.163] as well as of a Fe monolayer on W(100) (Fig. 24.12e) [24.164], and the out-of-plane orientation of a magnetic vortex core in the center of a nanomagnet exhibiting the flux closure configuration [24.165].

In addition, more complex atomic spin structures showing chiral or noncollinear arrangements have been identified [24.166-168]. Even the spin orientation of a single adatom could be detected, if the adatom is placed either directly on a ferromagnetic island [24.169] or close to a ferromagnetic stripe [24.26]. In the latter case, hysteresis curves of the ferromagnetic adatoms could be measured, as shown in Fig. 24.12fh. It was found that the adatoms couple either ferromagnetically (Fig. 24.12g) or antiferromagnetically (Fig. 24.12h) to the close-by magnetic stripe; i. e., the hysteresis is either in-phase or out-of-phase with the hysteresis of the stripe. This behavior, depending on adatom-stripe distance in an oscillating fashion, directly visualizes the famous Ruderman-Kittel-KasuyaYoshida (RKKY) interaction [24.26].

An interesting possibility of SP-STM is the observation of magnetodynamics on the nanoscale. Nanoscale ferromagnetic islands become unstable at a certain temperature, the so-called superparamagnetic tran- 


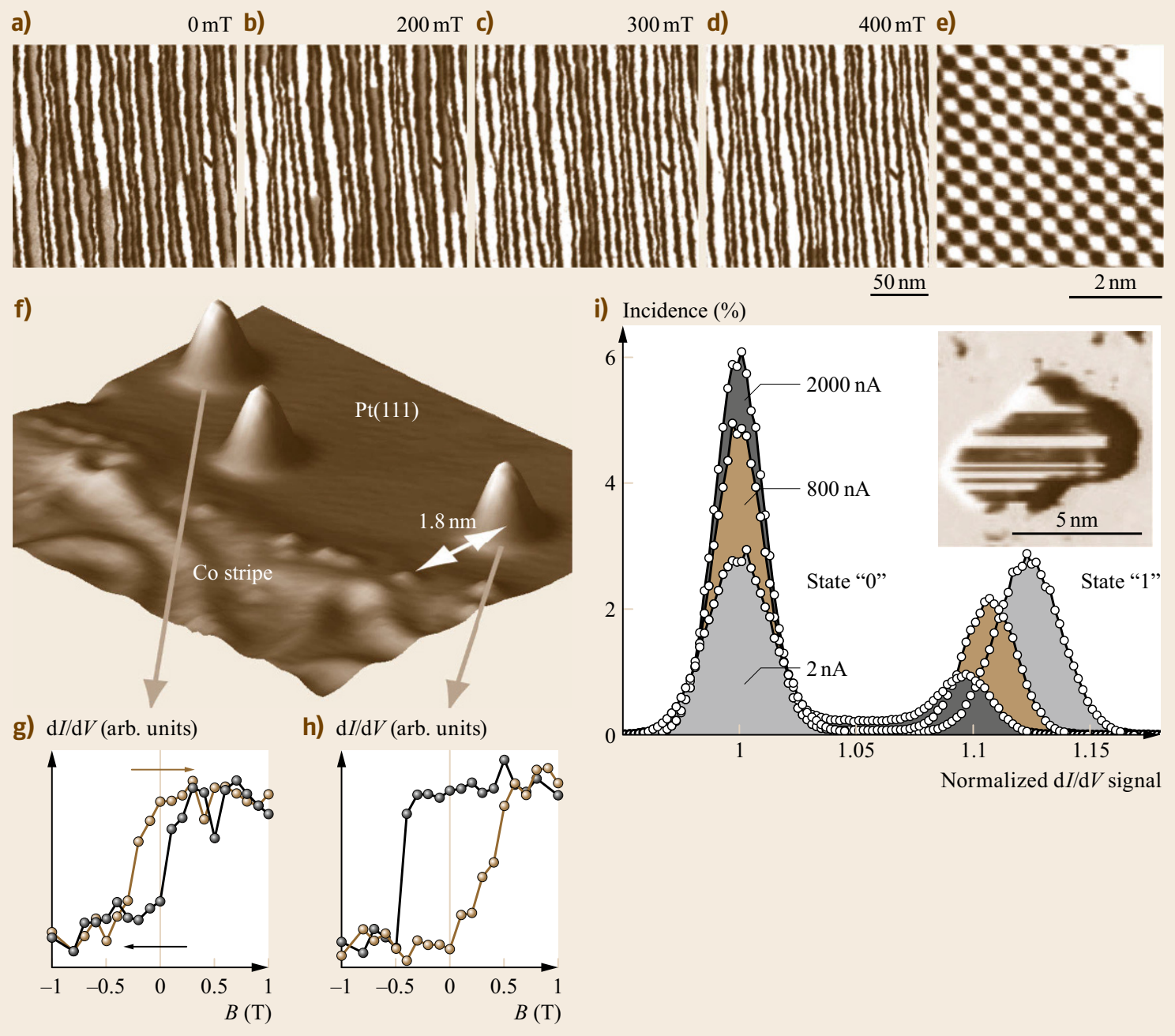

Fig. 24.12 (a-d) Spin-polarized STM images of 1.65 monolayers of Fe deposited on a stepped W(110) surface measured at different $B$ fields, as indicated. Double-layer and monolayer Fe stripes are formed on the W substrate; only the doublelayer stripes exhibit magnetic contrast with an out-of-plane sensitive tip, as used here. White and grey areas correspond to different domains. Note that more white areas appear with increasing field (@ M. Bode, Argonne (a-d)). (e) STM image of an antiferromagnetic Fe monolayer on W(001) exhibiting a checkerboard pattern of spin-down (dark) and spin-up (bright) atoms (C A. Kubetzka, Hamburg); (f) STM image of a $\operatorname{Pt}(111)$ surface with a Co stripe deposited at the Pt edge as marked. Single Co atoms, visible as three hills, are deposited subsequently on the surface at $T=25 \mathrm{~K}$; $(\mathrm{g}, \mathrm{h}) \mathrm{d} I / \mathrm{d} V(B)$ curves obtained above the Co atoms marked in (f) using a spin-polarized tip at $V=0.3 \mathrm{~V}$. The colors mark the sweeping direction of the $B$ field. Obviously the resulting contrast is hysteretic with $B$ and opposite for the two Co atoms. This indicates a different sign of ferromagnetic coupling to the Co stripe. (@ J. Wiebe, Hamburg (f-h)); (i) observed incidences of differential conductivities above a single monolayer Fe island on W(110) with a spin-polarized tip. The three curves are recorded at different tunneling currents and the increasing asymmetry shows a preferential spin direction with increasing spin-polarized current. Inset: $\mathrm{d} I / \mathrm{d} V$ image of the Fe island at $T=56 \mathrm{~K}$ showing the irregular change of $\mathrm{d} I / \mathrm{d} V$ intensity (C S. Krause, Hamburg)

sition temperature. Above this temperature, the direction of magnetization switches back and forth due to thermal excitations. This switching results in a stripelike contrast in SP-STM images, as visible in the in- set of Fig. 24.12i. The island appears dark during the time when the orientation of the island spin is opposite to the orientation of the tip spin, and switches to bright when the island spin orientation changes. By observing 
the switching as a function of time on different islands at different temperatures, the energy barriers of individual islands can be determined [24.170]. Even more importantly, the preferential orientation during switching can be tuned by the tunneling current. This is visible in Fig. 24.12i, which shows the measured orientational probability at different tunneling currents [24.171]. The observed asymmetry in the peak intensity increases with current, providing evidence that current-induced magnetization switching is possible even on the atomic scale.

\subsection{Scanning Force Microscopy and Spectroscopy}

The examples discussed in the previous section show the wide variety of physical questions that have been tackled with the help of LT-STM. Here, we turn to the other prominent scanning probe method that is applied at low temperatures, namely SFM, which gives complementary information on sample properties on the atomic scale.

The ability to detect forces sensitively with spatial resolution down to the atomic scale is of great interest, since force is one of the most fundamental quantities in physics. Mechanical force probes usually consist of a micromachined cantilever with a tip at its free end that is brought close to the sample surface. Basically, two methods exist to detect forces with cantilever-based probes: the static and the dynamic mode [24.172, 173]. They can be used to generate a laterally resolved image (microscopy mode) or to determine the distance dependence of force or a related quantity (spectroscopy mode)

In the static mode, a force that acts on the tip bends the cantilever. By measuring its deflection $\Delta z$, the tip-sample force $F_{\text {ts }}$ can be directly calculated from Hooke's law: $F_{\mathrm{ts}}=c_{\mathrm{z}} \Delta z$, where $c_{\mathrm{z}}$ denotes the spring constant of the cantilever. In the various dynamic modes, the cantilever is oscillated with amplitude $A$, typically at or near its eigenfrequency $f_{0}$. Under ambient conditions or in liquids, amplitude modulation (AMSFM) is typically used to detect amplitude changes or the phase shift between the driving excitation and cantilever oscillation. In vacuum, the frequency shift $\Delta f$ of the cantilever due to tip-sample interaction is measured by the frequency modulation technique (FMSFM). Converting the measured quantity (amplitude, phase or frequency shift) into a physically meaningful quantity, e.g., the tip-sample interaction force $F_{\text {ts }}$ or potential $V_{\mathrm{ts}}$, is not always straightforward and requires an analysis of the equation of motion of the oscillating tip [24.174-177]. Whatever method is used, the resolution of a cantilever-based force detection method is fundamentally limited by its intrinsic thermomechanical noise. If the cantilever is in thermal equilibrium at a temperature $T$, the equipartition theorem predicts a thermally induced root-mean-square (rms) motion of the cantilever in the $z$-direction of $z_{\mathrm{rms}}=\left(k_{\mathrm{B}} T / c_{\mathrm{eff}}\right)^{1 / 2}$, where $k_{\mathrm{B}}$ is the Boltzmann constant and $c_{\text {eff }}=c_{\mathrm{z}}+$ $\partial F_{\mathrm{ts}} / \partial z$. Note that usually $\partial F_{\mathrm{ts}} / \partial z \gg c_{\mathrm{z}}$ in the contact regime and $\partial F_{\mathrm{ts}} / \partial z<c_{\mathrm{z}}$ in the noncontact regime. Evidently, this fundamentally limits the force resolution in the static mode. Of course, the same is true for the different dynamic modes, because the thermal energy $k_{\mathrm{B}} T$ excites the eigenfrequency $f_{0}$ of the cantilever. Thermal noise is white noise, i. e., its spectral density is flat. However, if the cantilever transfer function is taken into account, one can see that the thermal energy mainly excites $f_{0}$. This explains the term thermo in thermomechanical noise, but what is the mechanical part?

A more detailed analysis reveals that the thermally induced cantilever motion is given by

$$
z_{\mathrm{rms}}=\sqrt{\frac{2 k_{\mathrm{B}} T B}{\pi c_{\mathrm{z}} f_{0} Q}},
$$

where $B$ is the measurement bandwidth and $Q$ is the quality factor of the cantilever. Analogous expressions can be obtained for all quantities measured in dynamic modes, because the deflection noise translates, e.g., into frequency noise [24.8]. Note that $f_{0}$ and $c_{\mathrm{z}}$ are correlated with each other via $2 \pi f_{0}=\left(c_{\mathrm{z}} / m_{\mathrm{eff}}\right)^{1 / 2}$, where the effective mass $m_{\text {eff }}$ depends on the geometry, the density, and the elasticity of the material. The $Q$-factor of the cantilever is related to the external damping of the cantilever motion in a medium and on the intrinsic damping within the material. This is the mechanical part of the fundamental cantilever noise. Thus, it becomes evident from (24.2) that the smallest fundamental noise is achievable in vacuum, where the $Q$-factors are more than 100 times larger than in air, and at low temperatures.

Besides the reduced noise, the application of force detection at low temperatures is motivated by increased stability and the possibility to observe phenomena that appear below a certain critical temperature $T_{\mathrm{c}}$, as outlined before. SFM experiments which have been performed at low temperatures until now were motivated by at least one of these reasons and have focused on atomicscale and intramolecular imaging, force spectroscopy, atomic and molecular manipulation as well as the investigation of electrostatic and magnetic properties at increasingly small length scales. In the following, we briefly describe some exemplary results from each area. 


\subsubsection{Atomic-Scale and Intramolecular Imaging}

In a simplified picture, the dimensions of the tip end and its distance to the surface limit the lateral resolution of force microscopy, since it is a near-field technique. Consequently, atomic resolution requires a stable single atom at the tip apex that has to be brought to a distance of some tenths of nanometers over an atomically flat surface. The latter condition can only be fulfilled in the dynamic mode, where the additional restoring force $c_{\mathrm{Z}} A$ at the lower turnaround point prevents the jump-to-contact phenomenon [24.19]. By preventing jump-to-contact, true atomic resolution is nowadays routinely obtained in vacuum (and increasingly, in liquids and air) by FM-SFM.

$\operatorname{Si}(111)-(7 \times 7)$ was the first surface on which true atomic resolution was achieved [24.178] and several studies have been performed at low temperatures on this well-known material [24.179-181]. Because of its importance to the microelectronics industry, the $\mathrm{Si}(100)$ surface has also been targeted by low-temperature FM-SFM measurements. In particular, measurements performed at low temperatures have shown that the structure of the surface is defined by buckled $\mathrm{Si}$ dimers with tilted bonds leading to a $c(4 \times 2)$ reconstruction [24.182]. Additionally, it has been determined that tip-induced interactions at small tip-sample distances lead to dimer flipping, resulting in the occasional observation of a $p(2 \times 1)$ reconstruction during low temperature experiments [24.183]. Moreover, experiments by Sweetman et al. have demonstrated deliberate flipping of single dimers on the $\mathrm{Si}(100)$ surface at low temperatures via controlled tip-sample interactions, corresponding to pure mechanical toggling of an atomicscale switch (Fig. 24.13) [24.184, 185]. Other low-temperature SFM experiments performed on semiconducting surfaces include those on indium arsenide, where noticeable displacements of tip and sample atoms due to interaction forces have been observed [24.186, 187]. Additionally, the combination of SFM and STM methods thanks to the utilization of tuning forks have allowed the precise determination of the surface structure of metal-semiconductor systems such as $\mathrm{Ag}: \mathrm{Si}(111)-$ $(\sqrt{3} \times \sqrt{3}) R 30^{\circ}$ at low temperatures [24.188].

As discussed before, a particular advantage associated with low-temperature SFM operation is reduced noise and increased thermal as well as mechanical stability, which allow atomic-resolution imaging of, e.g., weakly interacting van der Waals surfaces. For such surfaces, much smaller atomic corrugation amplitudes are expected compared with strongly interacting surfaces of, e.g., semiconductors. A typical example is

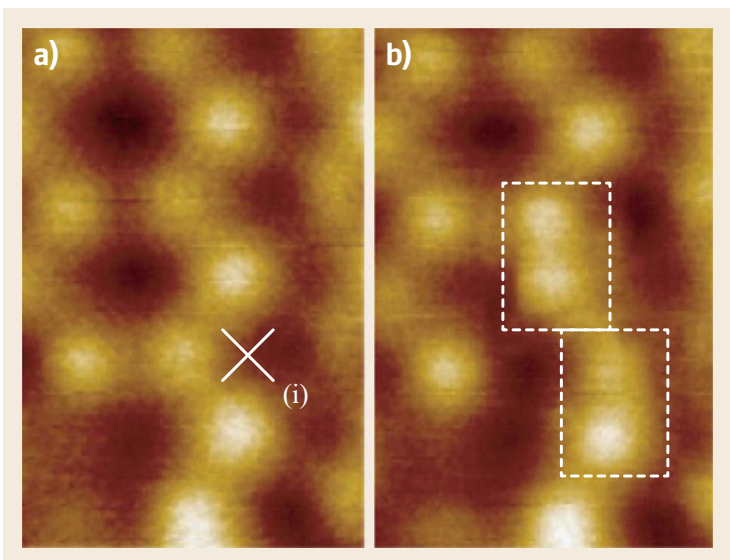

Fig. 24.13 LT-SFM images of the $\operatorname{Si}(100)$ surface that (a) demonstrate the atomic arrangement of the surface featuring the $c(4 \times 2)$ reconstruction and (b) dimer flipping (highlighted by dashed rectangles) induced by controlled force spectroscopy performed at location (i) in part (a). Adapted from [24.185]

graphite, a layered material, where the carbon atoms are covalently bonded and arranged in a honeycomb structure within the (0001) plane. Individual graphene layers stick together by van der Waals forces. Because of the $A B A$ stacking, three distinctive sites exist on the (0001) surface: carbon atoms with (A-type) and without ( $B$-type) a neighbor in the next graphite layer and the hollow site ( $H$-site) in the hexagon center. In static force microscopy operated in the contact regime as well as in STM, the contrast usually exhibits a trigonal symmetry with a periodicity of $246 \mathrm{pm}$, where $A$ - and $B$ site carbon atoms could not be distinguished. However, in high-resolution SFM images acquired at low temperatures, a large maximum and two different minima can be resolved [24.10, 189]. By comparison with other experimental and simulation results in the literature [24.190-192], the large maximum can be assigned to the $H$-site, while the two different minima represent A- and B-type carbon atoms. In addition to graphite, the atomic-scale structure of a pure van der Waals crystal consisting of individual xenon atoms could also be resolved by SFM performed at low temperatures, demonstrating the outstanding resolution and stability of the approach [24.193].

Of course, low-temperature SFM imaging experiments are not limited to semiconductor and van der Waals surfaces only. In particular, metal oxide surfaces such as $\mathrm{TiO}_{2}(110)[24.194,195]$ and ionic crystal surfaces such as $\operatorname{KBr}(001)$ [24.196] have also been imaged with atomic resolution at low temperatures, among others. 
Performing SFM experiments at low temperatures not only allows atomic-scale imaging of clean, atomically flat surfaces but may also remarkably lead to the detailed visualization of intramolecular structure of individual molecules adsorbed on surfaces [24.197-202] and even bonds between individual molecules [24.203]. The key factor enabling the associated high resolution is the selective and deliberate functionalization of the SFM probe tip by, typically, an individual CO molecule. While the exact physical mechanism that gives rise to the submolecular imaging capability (Pauli repulsion versus force-interaction-induced relaxations of the $\mathrm{CO}$ molecule dangling at the tip end) is still under debate [24.204, 205], high-resolution imaging with functionalized probes certainly represents the state-ofthe-art in low-temperature SFM experiments. In particular, while the visualization of the structural backbone of a single pentacene molecule [24.199] was covered worldwide by major media outlets (Fig. 24.14), the applications of the method rather quickly expanded and it recently became possible to precisely identify the structures of certain organic molecules [24.197, 200, 202], discriminate bond order [24.201], and perform KPFM with submolecular resolution [24.206, 207].

\subsubsection{Force Spectroscopy}

A wealth of information about the nature of the tipsample interaction can be obtained by measuring its distance dependence. This is usually done by recording the measured quantity (deflection, frequency shift, amplitude change, phase shift) while changing the tipsample distance by applying an appropriate voltage ramp to the $z$-electrode of the scan piezo. According to (24.2), low temperatures and high $Q$-factors (vacuum) considerably increase the force resolution. In the static mode, long-range forces and contact forces can be examined. Force measurements at small tip-sample distances are inhibited by the jump-to-contact phenomenon: If the force gradient $\partial F_{\mathrm{ts}} / \partial z$ becomes larger than the spring constant $c_{\mathrm{z}}$, the cantilever cannot resist the attractive tip-sample forces and the tip snaps into the surface. Sufficiently large spring constants prevent this effect, but reduce the force resolution. In the dynamic modes, as explained before, jump-to-contact can be avoided due to the additional restoring force $c_{\mathrm{z}} A$. The highest sensitivity can be achieved in vacuum by using the FM technique, i. e., by recording $\Delta f(z)$-curves. An alternative FM spectroscopy method, the recording of $\Delta f(A)$-curves, has been suggested in [24.208]. Note that if the amplitude is much larger than the characteristic decay length of the tip-sample force, the frequency shift cannot simply be converted into force gradients by using $\partial F_{\mathrm{ts}} / \partial z=2 c_{\mathrm{z}} \Delta f / f_{0}$ [24.209]. Several methods have been published to convert $\Delta f(z)$ data into the tip-sample force $F_{\mathrm{ts}}(z)$ and the tip-sample potential $V_{\mathrm{ts}}(z)$ [24.174-177]. In addition to reducing noise and increasing force resolution, a major benefit of perform-

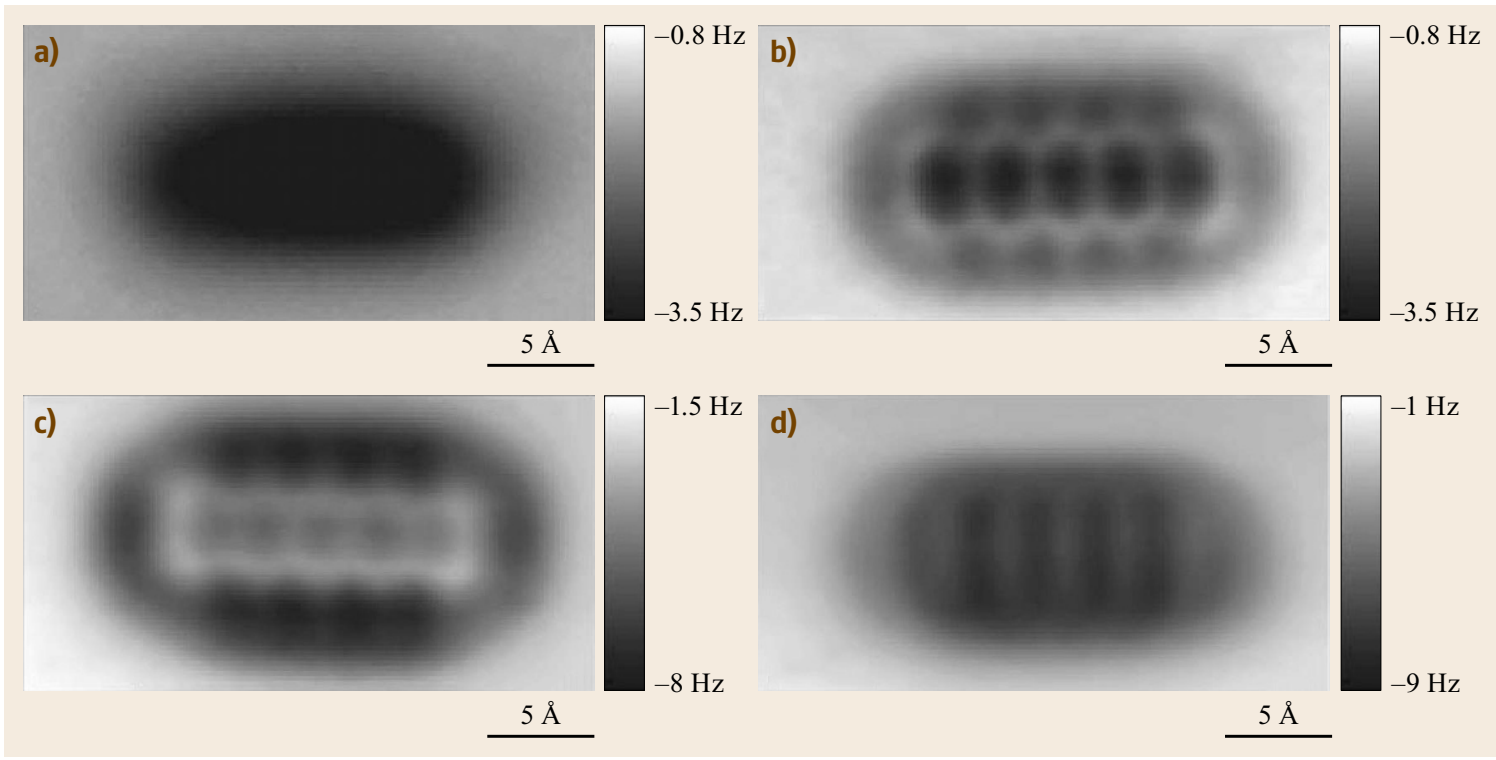

Fig. 24.14a-d Constant-height LT-SFM images of a single pentacene molecule acquired with (a) Ag-functionalized, (b) CO-functionalized, (c) Cl-functionalized, and (d) pentacene-functionalized tips. The CO-functionalized tip provides clear intramolecular resolution. The respective color bars indicate $\Delta f$ values. Adapted from [24.199]. Reprinted with permission from the AAAS 
ing force spectroscopy at low temperatures is drastically reduced thermal drift, which allows recording spectroscopy data on the same atomically defined surface site for extended periods of time.

Several research groups around the world have reported atomically resolved force spectroscopy results acquired at low temperatures not long after the first demonstration of atomic-resolution imaging via FM-SFM [24.191,210-213]. In particular, force spectroscopy data obtained on graphite has been found to be in good agreement with well-known interaction laws (Fig. 24.15a) [24.211]. Additionally, small differences in interaction forces exhibited by different surface atoms on $\mathrm{Si}(111)-(7 \times 7)$ have also been detected via low-temperature force spectroscopy [24.213]. Moreover, low-temperature force spectroscopy has been utilized on ionic crystal surfaces to, e.g., chemically identify tip and surface atoms as well as characterize elastic deformations and asymmetry artifacts [24.210, 212]. It was even shown that CO-terminated probes can be used to acquire several $\Delta f(z)$-curves above another $\mathrm{CO}$ molecule adsorbed on a $\mathrm{Cu}(111)$ surface at low temperatures, to obtain a two-dimensional, vertical map of interaction energy between the two molecules with high resolution (Fig. 24.15b) [24.214]. It should be mentioned that while spectacular results regarding chemical identification of individual atoms via force spectroscopy have been previously reported based on experiments performed at room temperature [24.215], the signal-to-noise-ratio had to be drastically increased by averaging about 100 curves at every atom species, which required an appropriate atom tracking scheme [24.6, 216].

While the pioneering experiments described above are all based on the careful acquisition of individual $\Delta f(z)$ curves at well-defined surface sites (or several such curves to form two-dimensional, vertical maps as in [24.214] and [24.218]) and eventual conversion to $F_{\mathrm{ts}}(z)$ or $V_{\mathrm{ts}}(z)$ data, it has recently been shown that it is possible to reliably combine thousands of such curves via the method of three-dimensional atomic force microscopy (3-D-AFM) to construct volumetric maps of interaction force/energy with $\mathrm{pm}$ spatial and $\mathrm{pN}$ (meV) force (energy) resolution [24.10, 194, 217, 219, 220], mainly thanks to the development of specialized data acquisition/processing procedures and operation at low temperatures which severely reduces thermal drift [24.221, 222]. Following the first proof-of-concept demonstration of 3-D-AFM on graphite [24.10], the method has been expanded to cover simultaneous acquisition of tunneling currents together with interaction forces. The corresponding technique of 3-D-AFM/STM (or alternatively, 3-D-SPM) has been recently shown to deliver complementary 3-D maps of interaction force

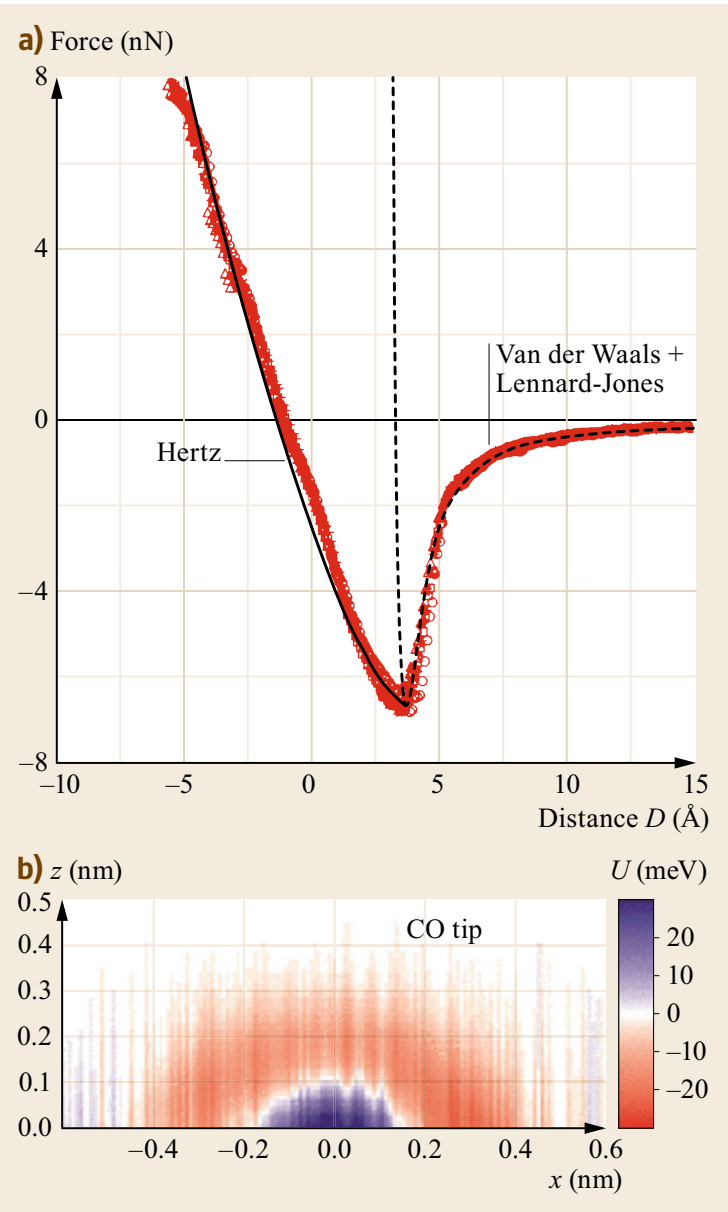

Fig. 24.15 (a) A force spectroscopy curve acquired on graphite with a silicon tip at low temperature. The interaction at first closely follows a combination of van der Waals and Lennard-Jones laws. At closer tip-sample distances, Hertzian contact mechanics approximates the force interaction reasonably well. (b) A two-dimensional map of interaction energy acquired with a CO-terminated tip on a $\mathrm{CO}$ molecule adsorbed on $\mathrm{Cu}(111)$. Red (blue) corresponds to attractive (repulsive) interactions. Adapted from [24.211] and [24.214], respectively

and tunneling current on sample surfaces of surfaceoxidized copper $(\mathrm{Cu}(100)-\mathrm{O})$ [24.217] and most recently, $\mathrm{TiO}_{2}$ (110) [24.194], with atomic resolution. Specifically, Fig. 24.16 shows the atomic structure associated with the $\mathrm{Cu}(100)-\mathrm{O}$ surface and the corresponding three-dimensional map of interaction forces spanning a lateral extent of $2.89 \mathrm{~nm} \times 2.89 \mathrm{~nm}$. The magnitude of the maximum interaction force measured in the bottom plane is $\approx 1.34 \mathrm{nN}$ whereas the contrast between atomically defined lattice sites is $\approx 23 \mathrm{pN}$. Moreover, a horizontal cut of interaction forces ex- 
tracted from the 3-D force map of Fig. 24.16b at the tip-sample distance of closest approach is shown in Fig. 24.17a, together with the simultaneously recorded map of tunneling currents at the same tip-sample distance (Fig. 24.17b). While symmetry arguments allow assigning the bright spots in the force image to individual oxygen atoms, the tunneling current map exposes a completely different structural arrangement that corresponds to the copper atoms of the surface. In addition to the simultaneous imaging of multiple atomic species on the sample surface, other exciting features of the discussed data set involve (i) the detection of minute differences in force interaction on individual oxygen atoms and (ii) the visualization and structural/chemical identification of surface defects, with support from $a b$ initio calculations [24.217]. To further illustrate the scientific insight that 3-D-AFM/STM measurements can provide, simultaneously acquired maps of interaction force and tunneling current on the prototypical metal oxide surface of $\mathrm{TiO}_{2}(110)$ are shown in Fig. 24.17c,d. In particular, it is observed that a peculiar defect in the form of a subsurface hydrogen atom modulates the surface density of electronic states, without a detectable influence on interaction force [24.194]. Thus, it is seen that the detailed information on tip-sample interactions that is obtained by 3-D-AFM/STM definitely has the potential to provide much needed experimental input into research fields as diverse as catalysis and friction, among others.

It must be mentioned that the application of the method of 3-D-AFM is not restricted to low temperatures and it has indeed been applied multiple times at room temperature thanks to the development of software-based drift correction methods [24.223, 224].
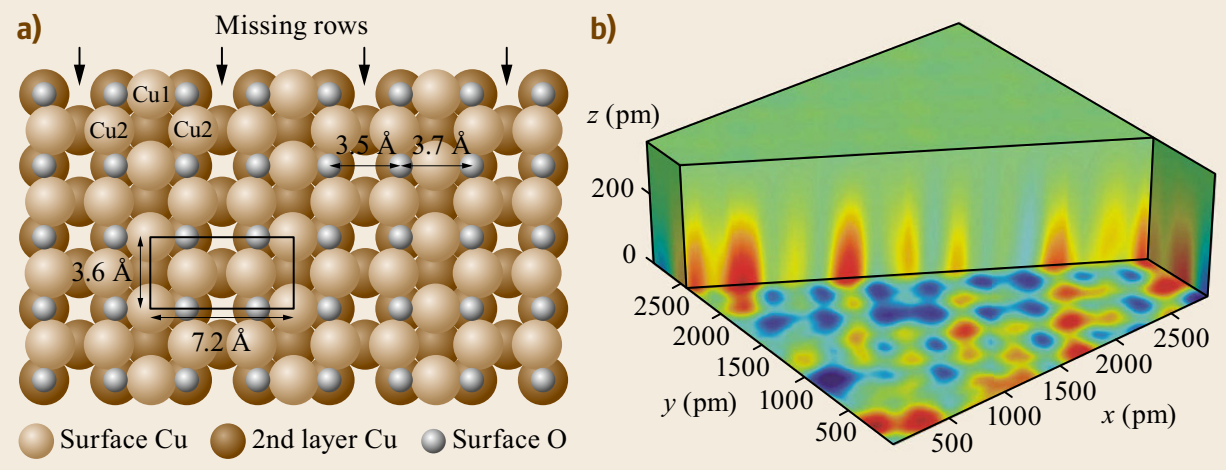

Fig. 24.16 (a) Structural model of the surface-oxidized $\mathrm{Cu}(100)$ surface $(\mathrm{Cu}(100)-\mathrm{O})$ ). (b) Atomically resolved, 3-D map of interaction forces acquired on $\mathrm{Cu}(100)-\mathrm{O}$ via 3-D-AFM at low temperature. Adapted from [24.217]
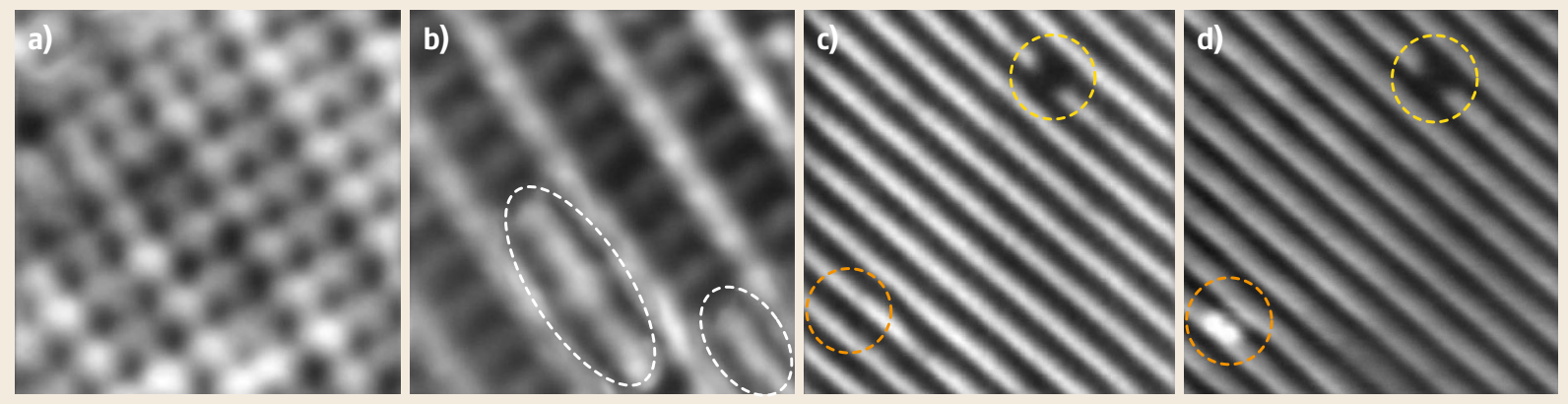

Fig. 24.17 (a,b) Simultaneously acquired, atomic-scale maps $(2.89 \mathrm{~nm} \times 2.89 \mathrm{~nm})$ of interaction force (a) and tunneling current (b) on the $\mathrm{Cu}(100)-\mathrm{O}$ surface at low temperature. Circular maxima in (a) correspond to oxygen atoms while the tunneling current channel (b) resolves copper atoms. Linear surface defects are highlighted by dashed white ellipses. (c,d) Simultaneously acquired, atomic-scale maps $(6.55 \mathrm{~nm} \times 6.55 \mathrm{~nm})$ of interaction force $(c)$ and tunneling current (d) on the $\mathrm{TiO}_{2}(110)$ surface at low temperature. Bright rows correspond to the arrangement of bridging oxygen ions on the surface. While a point defect in the form of an oxygen vacancy (dashed yellow circle) is visible in both data channels, a subsurface hydrogen interstitial (dashed orange circle) is found to modulate only the tunneling current data, without a detectable influence on interaction force. Adapted from [24.217] and [24.194], respectively 
On the other hand, low-temperature operation results in certain significant advantages that have been discussed in detail in the literature [24.222, 225]. Finally, the application of 3-D-AFM has recently expanded from atomically flat substrate surfaces to individual molecules [24.199, 226].

\subsubsection{Atomic and Molecular Manipulation}

Atomic-scale manipulation has first been demonstrated via the application of LT-STM and the method is still used successfully to manipulate individual adsorbates (atoms or molecules) on conducting surfaces (Sect. 24.3.1). In most of these experiments the adsorbate is dragged with the tip by the attractive force acting between the foremost tip apex atoms and the adsorbate. By adjusting a large or a small tip-sample distance via the tunneling resistance, it is possible to switch between imaging and manipulation. In the past decade, it has been shown that controlled manipulation of individual atoms and molecules is also possible by using dynamic SFM. Vertical manipulation was performed by pressing the tip in a controlled manner into a $\mathrm{Si}(111)-(7 \times 7)$ surface [24.227] at low temperatures. The strong repulsion leads to the removal of the selected silicon atom. The process could be traced by recording the frequency shift and the damping signal during the approach. For lateral manipulation, a rubbing technique has been utilized [24.228] again at low temperature - where the slow scan axis is halted above a selected atom, while the tip-surface distance is gradually reduced until the selected atom hops to a new stable position. Figure 24.18 shows a Ge adatom on $\mathrm{Ge}(111)-c(2 \times 8)$ that was moved during scanning in two steps from its original position (Fig. 24.18a) to its final position (Fig. 24.18c). More recently, vertical manipulation of individual $\mathrm{Cu}$ atoms on a reconstructed $\mathrm{Cu}(110): \mathrm{O}-p(2 \times 1)$ surface has been demonstrated at low temperature and the associated mechanism elucidated via a combined density functional theory and kinetic Monte Carlo simulation approach [24.229].

While it is not unreasonable to think that operation at low temperature would be a prerequisite for atomicscale manipulation due to the fact that the diffusion and desorption processes associated with the adsorbates would be severely suppressed, it has been shown that it is in fact possible to perform atomic-scale manipulation successfully at room temperature using dynamic SFM on semiconducting [24.230,231] and even insulating surfaces [24.232], leading to the ability to construct complex atomic-scale patterns on surfaces via a bottomup and deterministic approach (Fig. 24.19), with serious implications for atomic-scale device designs.

Atoms have not been the only species that have been controllably manipulated via SFM. In particular, it was shown a decade ago that individual, waterrelated defects can be manipulated on the insulating $\mathrm{CaF}_{2}(111)$ surface [24.233]. Additionally, individual molecules have also been laterally manipulated on various substrates by SFM [24.47,234-236] and even directed rotations of individual porphyrin molecules could be induced by precisely controlled force spectroscopy [24.237].

A major advantage of SFM compared to STM within the framework of atomic-scale manipulation is the fact that SFM allows researchers to measure vertical and lateral forces involved in the manipulation process. In a seminal paper published in 2008, Ternes et al. have manipulated individual $\mathrm{Co}$ atoms and $\mathrm{CO}$ molecules on $\mathrm{Pt}(111)$ and $\mathrm{Cu}(111)$ surfaces, and precisely measured the lateral forces required for manip- a)

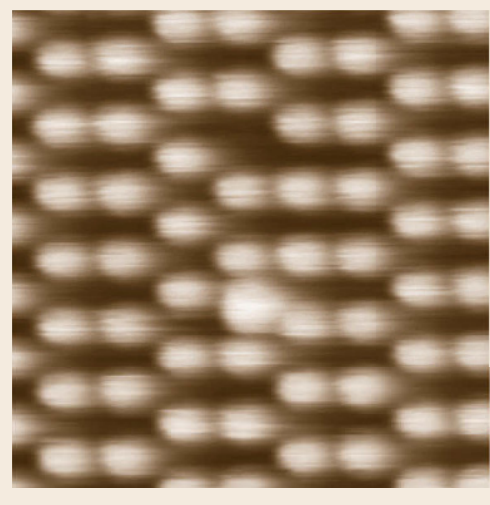

b)

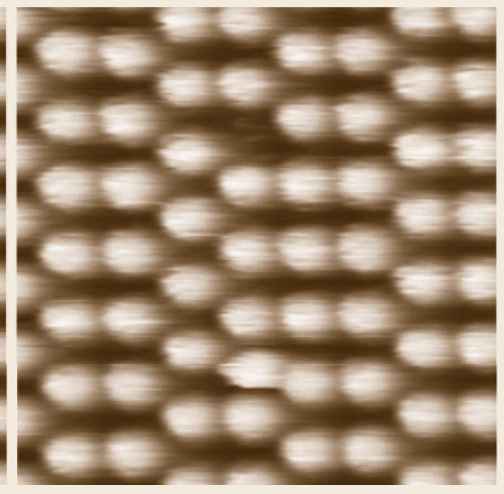

c)

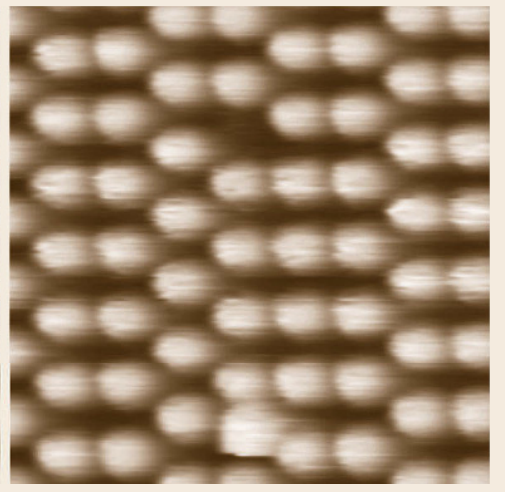

Fig. 24.18a-c Consecutively recorded FM-AFM images showing the tip-induced manipulation of a Ge adatom on $\mathrm{Ge}(111)-\mathrm{c}(2 \times 8)$ at $80 \mathrm{~K}$. Scanning was performed from bottom to top (C) N. Oyabu [24.228]) 


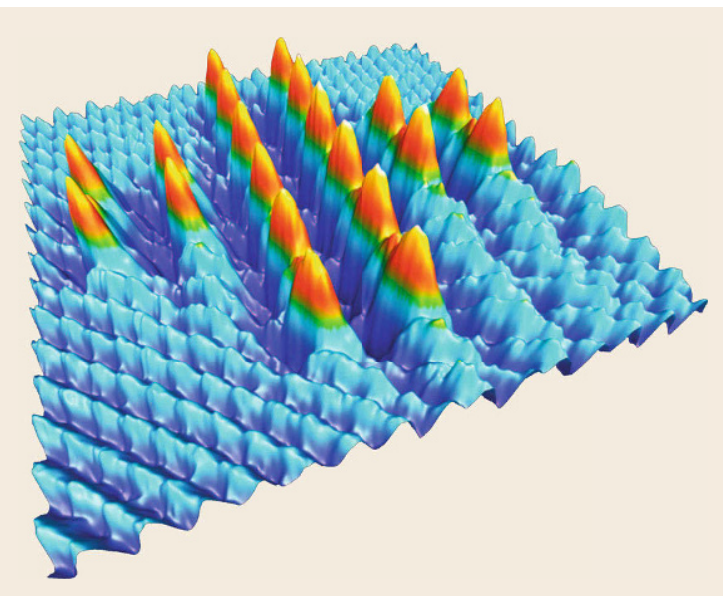

Fig. 24.19 Three-dimensional representation of a Swiss cross made of substitutional bromine ions that are assembled atom-by-atom on a sodium chloride surface via SFM. This result represents the first time that controllable atomic-scale manipulation on an insulator surface has been accomplished at room temperature. Adapted from [24.232]

ulation [24.47]. This goal was achieved by recording constant-height data at different tip-sample distances to obtain first $V_{\mathrm{ts}}(x, z)$ and then the lateral force $F_{\mathrm{x}}(x, z)=$ $\partial V_{\mathrm{ts}}(x, z) / \partial x$. Four curves of the whole data set are displayed in Fig. 24.20. The discontinuity of the lateral force at the lowest adjusted tip-sample distance $(z=160 \mathrm{pm})$ indicates the jump of a Co atom from one hollow site to the next on $\mathrm{Pt}(111)$ (see inset of Fig. 24.20). The jump takes place at about $210 \mathrm{pN}$, corresponding to the lateral force required for manipulation. More recently, Kawai et al. have performed a remarkable experiment where individual nanoribbons of graphene have been picked up from one side and controllably slid on a clean $\mathrm{Au}(111)$ surface via dynamic SFM at low temperature, whereby superlubric sliding was encountered [24.238].

\subsubsection{Kelvin Probe Force Microscopy}

The use of Kelvin probe force microscopy (KPFM) [24.239] in nanoscience and nanotechnology research has accelerated rapidly in recent years, thanks to the capability of the method to readily visualize local contact potential differences (LCPDs) over sample surfaces and even individual molecules with very high (down to atomic-scale and even intramolecular) resolution. The operational details of the method are explained elsewhere [24.240] and it should be sufficient here to state that KPFM is applied in conjunction with dynamic SFM, and the imaging typically involves a new feedback loop that is em-

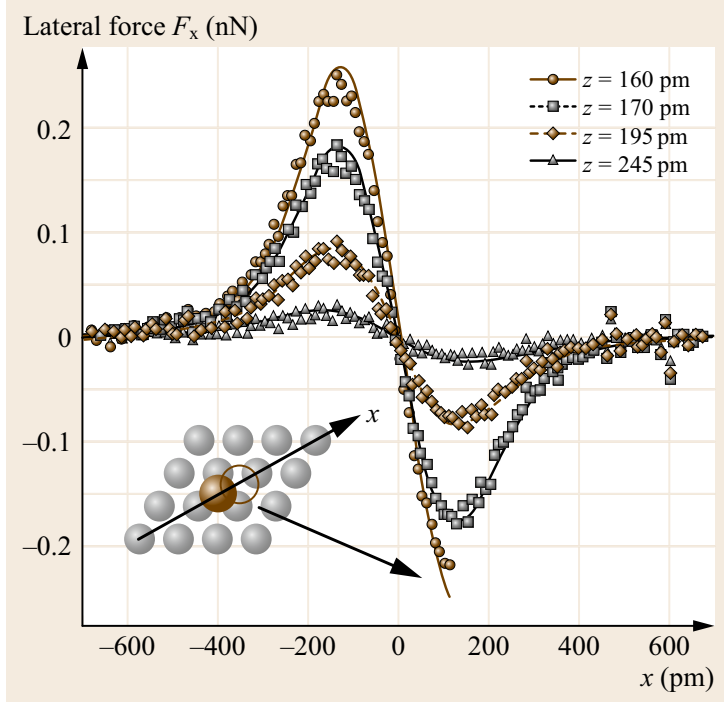

Fig. 24.20 Lateral force curves recorded at constant tipsample separation $z$. At the lowest separation a discontinuity appears, which marks the jump of the Co atom form one site to the next as indicated in the inset ( $($ ). M. Ternes [24.47])

ployed to determine the LCPD at each lateral position on a given sample surface by precisely measuring the bias voltage that needs to be applied for a minimization of the electrostatic tip-sample interaction. This leads to the simultaneous acquisition of a KPFM map together with the conventional topography map on the sample surface, whereby the KPFM map now reflects local LCPD variations on the surface.

While the mentioned variations in LCPD can be directly correlated to work function differences for conducting surfaces, in the case of insulating surfaces, KPFM contrast was shown to be determined by fixed charges and/or dipoles [24.240]. Even though the application of the KPFM method is not restricted to low temperatures, certain exciting results have been reported under such conditions. In particular, König et al. have determined the charge state of individual defects [24.241] and it was even possible to achieve submolecular resolution with KPFM and thus probe the charge distribution inside individual molecules at low temperatures $[24.206,207]$. Specifically, a COfunctionalized tip has been utilized by Mohn et al. to perform combined NC-AFM/KPFM (NC: non-contact) experiments on a single naphthalocyanine molecule, revealing an asymmetric charge distribution inside the molecule (Fig. 24.21) [24.206]. Thus, the capability of KPFM at low temperatures to provide essential quantitative information about the physical and chemical properties of individual adsorbates is demonstrated. 


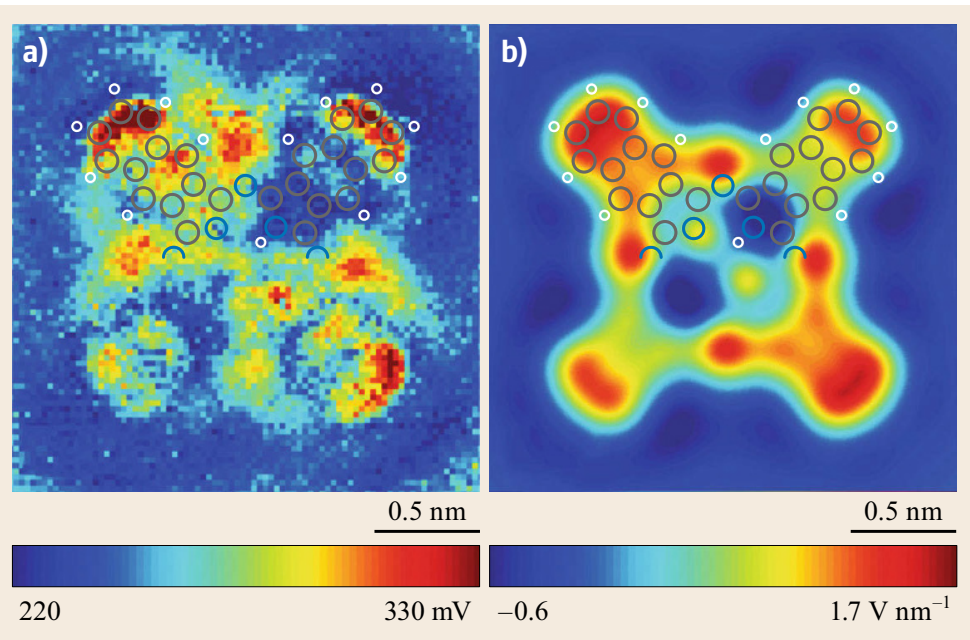

Fig. 24.21 (a) KPFM map of an individual naphthalocyanine molecule acquired at low temperature that highlights an asymmetric intramolecular charge distribution and (b) the corresponding calculation of electric field magnitude at a distance of $0.3 \mathrm{~nm}$ above the molecule. Adapted from [24.206]

\subsubsection{Magnetic Force Microscopy}

To detect magnetostatic tip-sample interactions with magnetic force microscopy (MFM), a ferromagnetic probe has to be used. Such probes are readily prepared by evaporating a thin magnetic layer, e.g., $10 \mathrm{~nm}$ iron, onto the tip. Because of the in-plane shape anisotropy of thin films, the magnetization of such tips lies predominantly along the tip axis, i. e., perpendicular to the surface. Since magnetostatic interactions are longrange, they can be separated from the topography by scanning at a certain constant height (typically around $20 \mathrm{~nm}$ ) above the surface, where the $z$-component of the sample stray field is probed (Fig. 24.22a). Therefore, MFM is always operated in the noncontact regime. The signal from the cantilever is directly recorded while the $z$-feedback is switched off. MFM can be operated in the static mode or in the dynamic modes (AM-MFM at ambient pressures and FM-MFM in vacuum; AM - amplitude-modulation; FM - frequency-modulation). A lateral resolution below $50 \mathrm{~nm}$ can be routinely obtained.

\section{Observation of Domain Patterns}

MFM is widely used to visualize domain patterns of ferromagnetic materials. At low temperatures, Moloni et al. observed the domain structure of magnetite below its Verwey transition temperature $\left(T_{\mathrm{V}}=\right.$ $122 \mathrm{~K}$ ) [24.242], but most of the work concentrated on thin films of $\mathrm{La}_{1-x} \mathrm{Ca}_{x} \mathrm{MnO}_{3}$ [24.243-245]. Below $T_{\mathrm{V}}$, the conductivity decreases by two orders of magnitude and a small structural distortion is observed. The domain structure of this mixed valence manganite is of great interest, because its resistivity strongly depends on the external magnetic field, i. e., it exhibits a large colossal magnetoresistive effect. To investigate the field dependence of domain patterns under ambient conditions, electromagnets have to be used. They can cause severe thermal drift problems due to Joule heating of the coils by large currents. Flux densities on the order of $100 \mathrm{mT}$ can be achieved. In contrast, much larger flux densities (more than $10 \mathrm{~T}$ ) can be rather easily produced by implementing a superconducting magnet in low-temperature setups. With such a design, Liebmann et al. recorded the domain structure along the major hysteresis loop of $\mathrm{La}_{0.7} \mathrm{Ca}_{0.3} \mathrm{MnO}_{3}$ epitaxially grown on $\mathrm{LaAlO}_{3}$ (Fig. 24.22b-f) [24.243]. The film geometry (with thickness of $100 \mathrm{~nm}$ ) favors an in-plane magnetization, but the lattice mismatch with the substrate induces an out-of-plane anisotropy. Thereby, an irregular pattern of strip domains appears at zero field. If the external magnetic field is increased, the domains with antiparallel orientation shrink and finally disappear in saturation (Fig. 24.22b,c). The residual contrast in saturation (Fig. 24.22d) reflects topographic features. If the field is decreased after saturation (Fig. 24.22e,f), cylindrical domains first nucleate and then start to grow. At zero field, the maze-type domain pattern evolves again. Such data sets can be used to analyze domain nucleation and the domain growth mode. Moreover, because of negligible drift at low temperatures, domain structure and surface morphology can be directly compared, because every MFM can be used as a regular topographyimaging force microscope.

\section{Detection of Individual Vortices in Superconductors}

Numerous low-temperature MFM experiments have been performed on superconductors [24.246-252]. Some basic features of superconductors have been mentioned already in Sect. 24.3.3, Superconductors. The main difference of STM/STS compared to MFM is its 


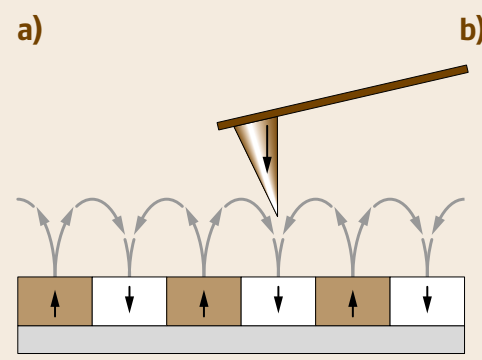

d)

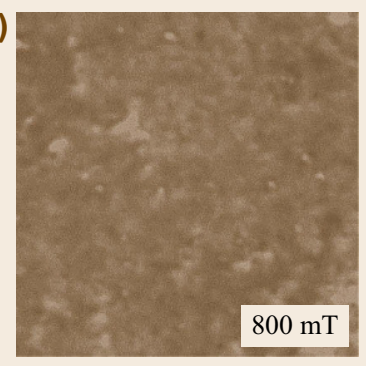

b)

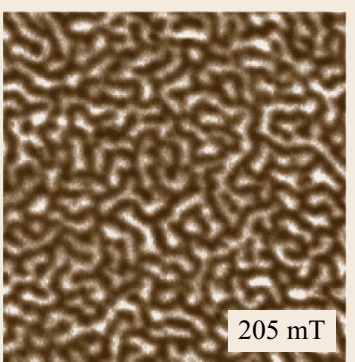

e)

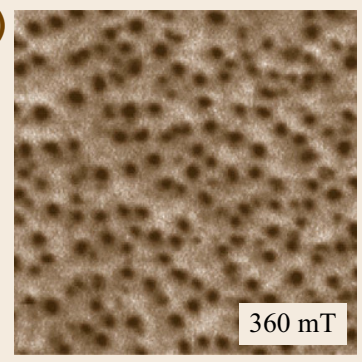

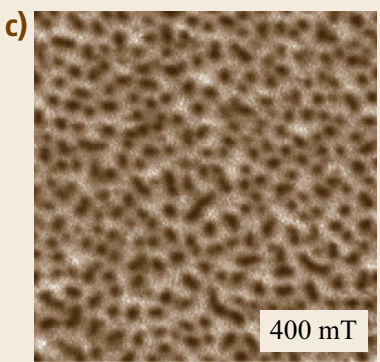

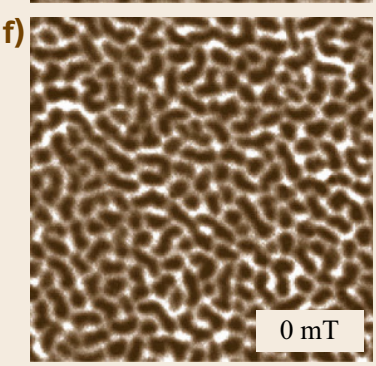

Fig. 24.22 Principle of MFM operation (a) and field-dependent domain structure of a ferromagnetic thin film (b-f) recorded at $5.2 \mathrm{~K}$ with FM-MFM. All images were recorded on the same $4 \mu \mathrm{m} \times 4 \mu \mathrm{m}$ scan area. The $\mathrm{La}_{0.7} \mathrm{Ca}_{0.3} \mathrm{MnO}_{3} / \mathrm{LaAlO}_{3}$ system exhibits a substrate-induced out-of-plane anisotropy. Bright and dark areas are visible and correspond to attractive and repulsive magnetostatic interactions, respectively. The series shows how the domain pattern evolves along the major hysteresis loop, i. e., from zero field to saturation at $600 \mathrm{mT}$ and back to zero field. (b-f) adapted from [24.243]

high sensitivity to the electronic properties of the surface. Therefore, careful sample preparation is a prerequisite. This is not so important for MFM experiments, since the tip is scanned at a certain distance above the surface.

Superconductors can be divided into two classes with respect to their behavior in an external magnetic field. For type-I superconductors, all magnetic flux is entirely excluded below their critical temperature $T_{\mathrm{c}}$ (Meissner effect), while for type-II superconductors, cylindrical inclusions (vortices) of normal material exist in a superconducting matrix (vortex state). The radius of the vortex core, where the Cooper pair density decreases to zero, is on the order of the coherence length $\xi$. Since the superconducting gap vanishes in the core, they can be detected by STS. Additionally, each vortex contains one magnetic quantum flux $\Phi=h / 2 e$ (where $h$ represents Planck's constant and $e$ the electron charge). Circular supercurrents around the core screen the magnetic field associated with a vortex; their radius is given by the London penetration depth $\lambda$ of the material. This magnetic field of the vortices can be detected by MFM. Investigations have been performed on the two most popular copper oxide high- $T_{\mathrm{c}}$ superconductors $\mathrm{YBa}_{2} \mathrm{Cu}_{3} \mathrm{O}_{7}$ [24.246, 252,253] and $\mathrm{Bi}_{2} \mathrm{Sr}_{2} \mathrm{CaCu}_{2} \mathrm{O}_{8}[24.247,252]$, on the only elemental conventional type-II superconductor $\mathrm{Nb}$ [24.249, 250], and on the layered compound crystal $\mathrm{NbSe}_{2}$ [24.250, 254].

Most often, vortices have been generated by cooling the sample from the normal state to below $T_{\mathrm{c}}$ in an external magnetic field. After such a field cooling procedure, the energetically most favorable vortex arrangement is a regular triangular Abrikosov lattice. Volodin et al. were able to observe such an Abrikosov lattice on $\mathrm{NbSe}_{2}$ [24.254]. The intervortex distance $d$ is related to the external field $B$ during cool-down via $d=(4 / 3)^{1 / 4}(\Phi / B)^{1 / 2}$. Another way to introduce vortices into a type-II superconductor is vortex penetration from the edge by applying a magnetic field at temperatures below $T_{\mathrm{c}}$. According to the Bean model, a vortex density gradient exists under such conditions within the superconducting material. $P i$ et al. slowly increased the external magnetic field until the vortex front approaching from the edge reached the scanning area [24.247].

If the vortex configuration is dominated by the pinning of vortices at randomly distributed structural defects, no Abrikosov lattice emerges. The influence of pinning centers can be studied easily by MFM, because every MFM can be used to scan the topography in its AFM mode. This has been done for natural growth defects by Moser et al. on $\mathrm{YBa}_{2} \mathrm{Cu}_{3} \mathrm{O}_{7}$ [24.253] and for $\mathrm{YBa}_{2} \mathrm{Cu}_{3} \mathrm{O}_{7}$ and niobium thin films, respectively, by Volodin et al. [24.251]. Roseman et al. investigated 
the formation of vortices in the presence of an artificial structure on niobium films [24.249], while $P i$ et al. produced columnar defects by heavy-ion bombardment in a $\mathrm{Bi}_{2} \mathrm{Sr}_{2} \mathrm{CaCu}_{2} \mathrm{O}_{8}$ single crystal to study the strong pinning at these defects [24.247].

Figure 24.23 demonstrates that MFM is sensitive to the polarity of vortices. In Fig. 24.23a, six vortices have been produced in a niobium film by field cooling in $+0.5 \mathrm{mT}$. The external magnetic field and tip magnetization are parallel, therefore, the tip-vortex interaction is attractive (bright contrast). To remove the vortices, the niobium was heated above $T_{\mathrm{c}}(\approx 9 \mathrm{~K})$. Thereafter, vortices of opposite polarity have been produced by field cooling in $-0.5 \mathrm{mT}$, which appear dark in Fig. 24.23b. The vortices are probably bound to strong pinning sites, because the vortex positions are identical in both images of Fig. 24.23. By imaging the vortices at different scanning heights, Roseman et al. tried to extract values for the London penetration depth from the scan-height dependence of their profiles [24.248]. While good qualitative agreement with theoretical predictions has been found, the absolute values do not agree with published literature values. The disagreement was attributed to the convolution between the tip and vortex stray fields. Better values might be obtained with calibrated tips.

\subsubsection{Magnetic Exchange Force Microscopy and Spectroscopy}

The resolution of MFM is limited to the nanometer range, because the long-range magnetostatic tipsample interaction is not localized between individual surface atoms and the foremost tip apex atom [24.255].

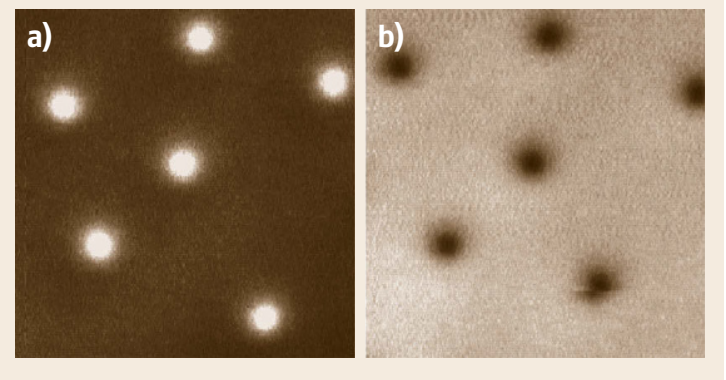

Fig. 24.23 Two $5 \mu \mathrm{m} \times 5 \mu \mathrm{m}$ FM-MFM images of vortices in a niobium thin film after field-cooling at $0.5 \mathrm{mT}$ (a) and $-0.5 \mathrm{mT}(\mathrm{b})$, respectively. Since the external magnetic field was parallel in (a) and antiparallel in (b) with respect to the tip magnetization, the vortices exhibit opposite contrast. Strong pinning dominates the position of the vortices, since they appear at identical locations in (a) and (b) and are not arranged in a regular Abrikosov lattice (C) P. Grütter [24.248])
As early as 1991 Wiesendanger et al. [24.256] proposed that the short-range magnetic exchange interaction can be utilized to image the configuration of magnetic moments with atomic resolution. For the suggested test system - $\mathrm{NiO}(001)$, an antiferromagnetic insulator Momida and Oguchi provided density functional calculations [24.257]. They found a magnetic exchange force between the magnetic moments of a single iron atom (the tip) and nickel surface atoms of more than $0.1 \mathrm{nN}$ at tip-sample distances below $0.5 \mathrm{~nm}$. Eventually, Kaiser et al. were able to prove the feasibility of magnetic exchange force microscopy (MExFM) on $\mathrm{NiO}(001)$ [24.258]. The super-exchange between neighboring (111) planes via bridging oxygen atoms results in a row-wise antiferromagnetic configuration of magnetic moments on the (001) surface. Hence the magnetic surface unit cell is twice as large as the chemical surface unit cell. Figure 24.24a shows the atomicscale contrast due to a pure chemical interaction. Maxima and minima correspond to the oxygen and nickel atoms, respectively. Their arrangement represents the $(1 \times 1)$ surface unit cell. Figure $24.24 \mathrm{~b}$ exhibits an additional modulation on chemically and structurally equivalent rows of nickel atoms (the minima). The structure corresponds to the $(2 \times 1)$ magnetic surface unit cell. Since the spin-carrying nickel $3 \mathrm{~d}$ states are highly localized, the magnetic contrast only becomes significant at very small tip-sample distances. Subsequently, Schmidt
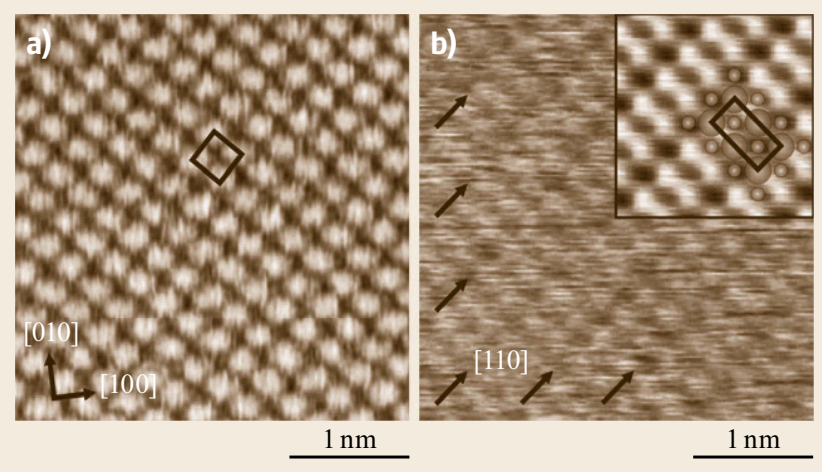

Fig. 24.24 (a) Pure chemical contrast on $\mathrm{NiO}(001)$ obtained with AFM using a nonmagnetic tip. Oxygen and nickel atoms are represented as maxima and minima, respectively, forming the $(1 \times 1)$ surface unit cell (black square). Arrows indicate the main crystallographic directions. (b) Additional modulation on neighboring nickel rows along the [110] direction (see arrows) due to the magnetic exchange interaction obtained with MExFM using a magnetic tip. The $(2 \times 1)$ structure (black rectangle in the inset) represents the magnetic surface unit cell. The inset is tiled together from the averaged magnetic unit cell calculated from the raw data, whereby the signal-to-noise ratio is significantly increased. Adapted from [24.258] 


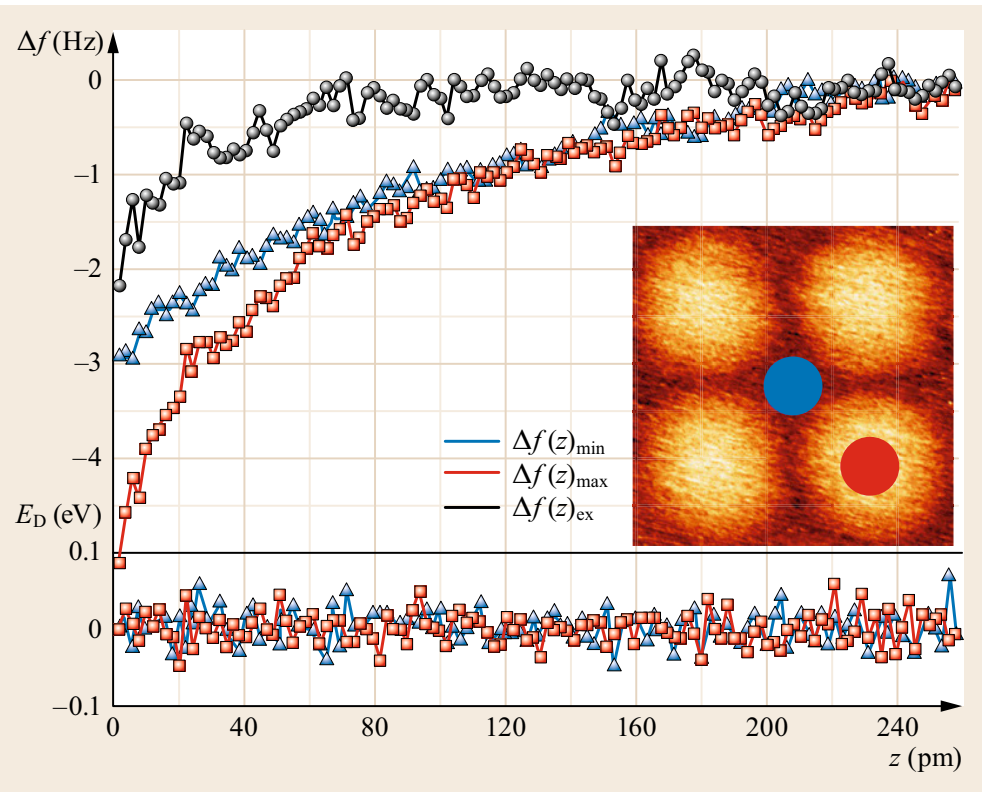

Fig. 24.25 Individual force spectroscopy curves acquired on two iron atoms with opposite spin (blue and red, respectively) and the corresponding difference (black) which is directly related to the magnetic exchange interaction. The curves in the bottom panel highlight the use of a nondissipative tip (energy dissipation $E_{\mathrm{D}} \approx 0$ ). Adapted from [24.259] et al. were able to perform MExFM with a much better signal-to-noise ratio on an itinerant metallic system: the antiferromagnetic iron monolayer on W(001) [24.260]. Density functional theory performed with a realistic tip model indicated significant relaxations of tip and sample atoms during imaging. Moreover, a comparison between simulation and experimental data revealed complex interplay between chemical and magnetic interaction, which results in the observed atomic-scale contrast. More recently, it became possible to quantify the distance dependence of the magnetic exchange interaction on metallic systems using magnetic exchange force spectroscopy (MExFS) [24.259] that relies on three-dimensional recording of interaction forces (Sect. 24.4.2). Specifically, force spectroscopy data obtained on atoms with opposite spin are subtracted from each other and the result corresponds to the magnetic exchange interaction (Fig. 24.25). Moreover, controllable magnetization switching via the utilization of the magnetic exchange interaction during MExFS experiments has been demonstrated [24.261].

\subsubsection{Magnetic Resonance Force Microscopy}

Another ambitious force-based approach utilizing magnetic tips is the proposed detection of individual spins by magnetic resonance force microscopy (MRFM) [24.262, 263]. Conventionally, nuclear spins are investigated by nuclear magnetic resonance (NMR), a spectroscopic technique to obtain microscopic chemical and physical information about molecules. An im- portant application of NMR for medical diagnostics is magnetic resonance imaging (MRI). This tomographic imaging technique uses the NMR signal from thin slices through, e.g., the human body, to reconstruct its threedimensional structure. Currently, at least $10^{12}$ nuclear spins must be present in a given volume to obtain a significant MRI signal. The ultimate goal of MRFM is to combine aspects of force microscopy with MRI to achieve true 3-D imaging with atomic resolution and elemental selectivity.

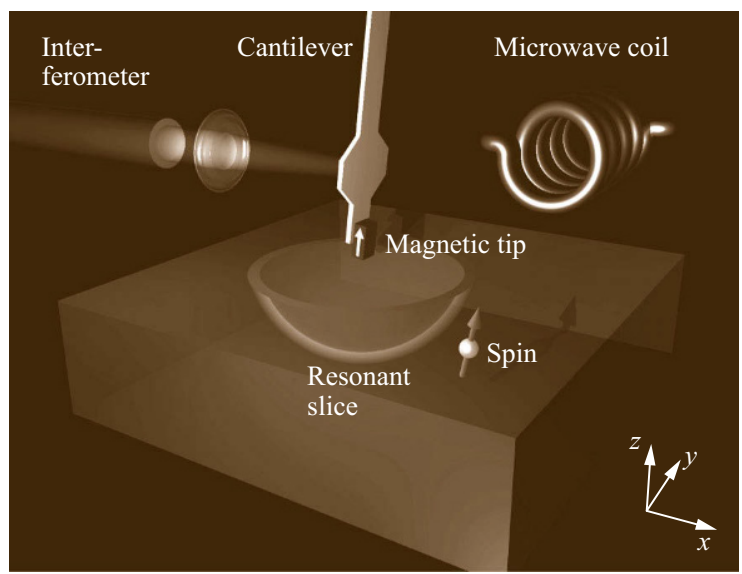

Fig. 24.26 MRFM setup. The cantilever with the magnetic tip oscillates parallel to the surface. Only electron spins within a hemispherical slice, where the stray field of the tip plus the external field matches the condition for magnetic resonance, can contribute to the MRFM signal due to cyclic spin inversion (C D. Rugar [24.264]) 
The experimental setup is sketched in Fig. 24.26. An oscillating cantilever with a magnetic tip at its end points toward the surface. The spherical resonant slice within the sample represents those points where the stray field from the tip and the external field match the condition for magnetic resonance. The cyclic spin flip causes a slight shift of the cantilever frequency due to the magnetic force exerted by the spin on the tip. Since the forces are extremely small, very low temperatures are required.

To date, no individual nuclear spins have been detected by MRFM. However, the design of ultrasensitive cantilevers made considerable progress, and the detection of forces below $1 \times 10^{-18} \mathrm{~N}$ has been achieved [24.265]. Thereby, it became possible to perform nuclear magnetic resonance [24.266] and ferromagnetic resonance [24.267] experiments of spin ensembles with micrometer resolution. Moreover, in $\mathrm{SiO}_{2}$ the magnetic moment of a single electron, which is three orders of magnitude larger than the nuclear magnetic moment, could be detected by $R u$ gar et al. using the setup shown in Fig. 24.26 at $1.6 \mathrm{~K}$ [24.264]. This major breakthrough demonstrates the capability of force microscopy to detect single spins. More recently, Degen et al. have combined MRFM with 3-D image reconstruction to enable MRI on biological samples with a spatial resolution of $\approx$ $10 \mathrm{~nm}$ [24.268].

\subsection{Summary}

It was the intention of this chapter to give an introduction to the application of scanning probe microscopy at low temperatures. The first three sections were devoted to a general introduction, an analysis of the specialities of microscope operation at low temperatures, and an overview of related instrumentation. It was emphasized that low-temperature operation of SPMs can significantly increase both the achievable resolution and the overall stability of the instrument.

The goal of the subsequent sections was to illustrate some of the most active areas of application for LT-SPM. In particular, LT-STM allows the controlled manipulation of individual atoms and the characteriza-

tion of electronic properties with atomic specificity. On essentially the same scale, LT-SFM allows the measurement of different types of force fields that provide complementary information. Nevertheless, the provided list of applications is certainly not complete, as this would go far beyond the scope of this handbook chapter.

In summary, the wide variety of associated techniques as well as the many physical questions which can be tackled by LT-SPM make it an unprecedented tool for nanotechnology. And despite the many investigations using LT-SPM that have already been performed, the authors believe that there is still plenty of room at the bottom [24.269].

\section{References}

24.1 G. Binnig, H. Rohrer: Scanning tunneling microscopy, Helvetica Physica Acta 55, 726-735 (1982)

24.2 R. Wiesendanger: Scanning Probe Microscopy and Spectroscopy (Cambridge Univ. Press, Cambridge 1994)

24.3 D.A. Bonnell, D.N. Basov, M. Bode, U. Diebold, S.V. Kalinin, V. Madhavan, L. Novotny, M. Salmeron, U.D. Schwarz, P.S. Weiss: Imaging physical phenomena with local probes: From electrons to photons, Rev. Modern Phys. 84, 1343-1381 (2012)

24.4 M. Tinkham: Introduction to Superconductivity (McGraw-Hill, New York 1996)

24.5 J. Kondo: Theory of dilute magnetic alloys, Solid State Phys. 23, 183-281 (1969)

24.6 M. Abe, Y. Sugimoto, 0. Custance, S. Morita: Room-temperature reproducible spatial force spectroscopy using atom-tracking technique, Appl. Phys. Lett. 87, 173503 (2005)
24.7 M. Abe, Y. Sugimoto, T. Namikawa, K. Morita, N. Oyabu, S. Morita: Drift-compensated data acquisition performed at room temperature with frequency modulation atomic force microscopy, Appl. Phys. Lett. 90, 203103 (2007)

24.8 T.R. Albrecht, P. Grutter, D. Horne, D. Rugar: Frequency modulation detection using high- $Q$ cantilevers for enhanced force microscope sensitivity, J. Appl. Phys. 69, 668-673 (1991)

24.9 F.J. Giessibl, H. Bielefeldt, S. Hembacher, J. Mannhart: Calculation of the optimal imaging parameters for frequency modulation atomic force microscopy, Appl. Surf. Sci. 140, 352-357 (1999)

24.10 B.J. Albers, T.C. Schwendemann, M.Z. Baykara, N. Pilet, M. Liebmann, E.I. Altman, U.D. Schwarz: Three-dimensional imaging of short-range chemical forces with picometre resolution, Nat. Nanotechnol. 4, 307-310 (2009) 
24.11 M. Morgenstern, D. Haude, V. Gudmundsson, C. Wittneven, R. Dombrowski, R. Wiesendanger: Origin of Landau oscillations observed in scanning tunneling spectroscopy on $n-\operatorname{InAs}(110)$, Phys. Rev. B 62, 7257-7263 (2000)

24.12 B. Uluutku, M.Z. Baykara: Effect of lateral tip stiffness on atomic-resolution force field spectroscopy, J. Vac. Sci. Technol. B 31, 041801 (2013)

24.13 B. Uluutku, M.Z. Baykara: Artifacts related to tip asymmetry in high-resolution atomic force microscopy and scanning tunneling microscopy measurements of graphitic surfaces, J. Vac. Sci. Technol. B 33, 031802 (2015)

24.14 D.M. Eigler, P.S. Weiss, E.K. Schweizer, N.D. Lang: Imaging Xe with a low-temperature scanning tunneling microscope, Phys. Rev. Lett. 66, 11891192 (1991)

24.15 P.S. Weiss, D.M. Eigler: Site dependence of the apparent shape of a molecule in scanning tunneling micoscope images: Benzene on Pt $\{111\}$, Phys. Rev. Lett. 71, 3139-3142 (1993)

24.16 D.M. Eigler, E.K. Schweizer: Positioning single atoms with a scanning tunnelling microscope, Nature 344, 524-526 (1990)

24.17 H.J. Hug, B. Stiefel, P.J.A. van Schendel, A. Moser, S. Martin, H.J. Guntherodt: A low temperature ultrahigh vacuum scanning force microscope, Rev. Sci. Instrum. 70, 3625-3640 (1999)

24.18 B.J. Albers, M. Liebmann, T.C. Schwendemann, M.Z. Baykara, M. Heyde, M. Salmeron, E.I. Altman, U.D. Schwarz: Combined low-temperature scanning tunneling/atomic force microscope for atomic resolution imaging and site-specific force spectroscopy, Rev. Sci. Instrum. 79, 033704 (2008)

24.19 F.J. Giessibl: Advances in atomic force microscopy, Rev. Modern Phys. 75, 949-983 (2003)

24.20 G. Dujardin, R.E. Walkup, P. Avouris: Dissociation of individual molecules with electrons from the tip of a scanning tunneling microscope, Science 255, 1232-1235 (1992)

24.21 H.J. Lee, W. Ho: Single-bond formation and characterization with a scanning tunneling microscope, Science 286, 1719-1722 (1999)

24.22 J. Repp, G. Meyer, F.E. Olsson, M. Persson: Controlling the charge state of individual gold adatoms, Science 305, 493-495 (2004)

24.23 R. Berndt, R. Gaisch, J.K. Gimzewski, B. Reihl, R.R. Schlittler, W.D. Schneider, M. Tschudy: Photon emission at molecular resolution induced by a scanning tunneling microscope, Science 262, 1425-1427 (1993)

24.24 X.H. Qiu, G.V. Nazin, W. Ho: Vibrationally resolved fluorescence excited with submolecular precision, Science 299, 542-546 (2003)

24.25 B.G. Briner, M. Doering, H.P. Rust, A.M. Bradshaw: Microscopic molecular diffusion enhanced by adsorbate interactions, Science 278, 257-260 (1997)

24.26 F. Meier, L. Zhou, J. Wiebe, R. Wiesendanger: Revealing magnetic interactions from single-atom magnetization curves, Science 320, 82-86 (2008)

24.27 S. Loth, M. Etzkorn, C.P. Lutz, D.M. Eigler, A.J. Heinrich: Measurement of fast electron spin relaxation times with atomic resolution, Science 329, 1628-1630 (2010)

24.28 F. Donati, S. Rusponi, S. Stepanow, C. Waeckerlin, A. Singha, L. Persichetti, R. Baltic, K. Diller, F. Patthey, E. Fernandes, J. Dreiser, Z. Sljivancanin, K. Kummer, C. Nistor, P. Gambardella, H. Brune: Magnetic remanence in single atoms, Science 352, 318-321 (2016)

24.29 J. Kliewer, R. Berndt, E.V. Chulkov, V.M. Silkin, P.M. Echenique, S. Crampin: Dimensionality effects in the lifetime of surface states, Science $\mathbf{2 8 8}$, 1399-1402 (2000)

24.30 M.F. Crommie, C.P. Lutz, D.M. Eigler: Imaging standing waves in a two-dimensional electron gas, Nature 363, 524-527 (1993)

24.31 H. Gawronski, M. Mehlhorn, K. Morgenstern: Imaging phonon excitation with atomic resolution, Science 319, 930-933 (2008)

24.32 A.J. Heinrich, J.A. Gupta, C.P. Lutz, D.M. Eigler: Single-atom spin-flip spectroscopy, Science 306, 466-469 (2004)

24.33 B.C. Stipe, M.A. Rezaei, W. Ho: Single-molecule vibrational spectroscopy and microscopy, Science 280, 1732-1735 (1998)

24.34 C.W.J. Beenakker, H. Vanhouten: Quantum transport in semiconductor nanostructures, Solid State Phys. 44, 1-228 (1991)

24.35 K.K. Gomes, A.N. Pasupathy, A. Pushp, S. Ono, Y. Ando, A. Yazdani: Visualizing pair formation on the atomic scale in the high-T-c superconductor $\mathrm{Bi}_{2} \mathrm{Sr}_{2} \mathrm{CaCu}_{2} \mathrm{O}_{8+\delta}$, Nature 447, 569-572 (2007)

24.36 K.M. Lang, V. Madhavan, J.E. Hoffman, E.W. Hudson, H. Eisaki, S. Uchida, J.C. Davis: Imaging the granular structure of high-T-c superconductivity in underdoped $\mathrm{Bi}_{2} \mathrm{Sr}_{2} \mathrm{CaCu}_{2} \mathrm{O}_{8+\delta}$, Nature 415, 412416 (2002)

24.37 J. Lee, K. Fujita, K. McElroy, J.A. Slezak, M. Wang, Y. Aiura, H. Bando, M. Ishikado, T. Masui, J.X. Zhu, A.V. Balatsky, H. Eisaki, S. Uchida, J.C. Davis: Interplay of electron-lattice interactions and superconductivity in $\mathrm{Bi}_{2} \mathrm{Sr}_{2} \mathrm{CaCu}_{2} \mathrm{O}_{8+\delta}$, Nature 442, 546-550 (2006)

24.38 R.S. Becker, J.A. Golovchenko, B.S. Swartzentruber: Atomic-scale surface modifications using a tunnelling microscope, Nature 325, 419-421 (1987)

24.39 P.G. Piva, G.A. DiLabio, J.L. Pitters, J. Zikovsky, M. Rezeq, S. Dogel, W.A. Hofer, R.A. Wolkow: Field regulation of single-molecule conductivity by a charged surface atom, Nature 435, 658-661 (2005)

24.40 J.A. Stroscio, D.M. Eigler: Atomic and molecular manipulation with the scanning tunneling microscope, Science 254, 1319-1326 (1991)

24.41 A.J. Heinrich, C.P. Lutz, J.A. Gupta, D.M. Eigler: Molecule cascades, Science 298, 1381-1387 (2002)

24.42 L. Bartels, G. Meyer, K.H. Rieder: Basic steps of lateral manipulation of single atoms and diatomic clusters with a scanning tunneling microscope tip, Phys. Rev. Lett. 79, 697-700 (1997)

24.43 J.J. Schulz, R. Koch, K.H. Rieder: New mechanism for single atom manipulation, Phys. Rev. Lett. 84, 
4597-4600 (2000)

24.44 J.A. Stroscio, R.J. Celotta: Controlling the dynamics of a single atom in lateral atom manipulation, Science 306, 242-247 (2004)

24.45 M. Lastapis, M. Martin, D. Riedel, L. Hellner, G. Comtet, G. Dujardin: Picometer-scale electronic control of molecular dynamics inside a single molecule, Science 308, 1000-1003 (2005)

24.46 J.A. Stroscio, F. Tavazza, J.N. Crain, R.J. Celotta, A.M. Chaka: Electronically induced atom motion engineered CoCun nanostructures, Science 313, 948-951 (2006)

24.47 M. Ternes, C.P. Lutz, C.F. Hirjibehedin, F.J. Giessibl, A.J. Heinrich: The force needed to move an atom on a surface, Science 319, 1066-1069 (2008)

24.48 J.I. Pascual, N. Lorente, Z. Song, H. Conrad, H.P. Rust: Selectivity in vibrationally mediated single-molecule chemistry, Nature 423, 525-528 (2003)

24.49 T.C. Shen, C. Wang, G.C. Abeln, J.R. Tucker, J.W. Lyding, P. Avouris, R.E. Walkup: Atomic-scale desorption through electronic and vibrational excitation mechanisms, Science 268, 1590-1592 (1995)

24.50 T. Komeda, Y. Kim, M. Kawai, B.N.J. Persson, H. Ueba: Lateral hopping of molecules induced by excitation of internal vibration mode, Science 295, 2055-2058 (2002)

24.51 Y.W. Mo: Reversible rotation of antimony dimers on the silicon (001) surface with a scanning tunneling microscope, Science 261, 886-888 (1993)

24.52 B.C. Stipe, M.A. Rezaei, W. Ho: Inducing and viewing the rotational motion of a single molecule, Science 279, 1907-1909 (1998)

24.53 P. Liljeroth, J. Repp, G. Meyer: Current-induced hydrogen tautomerization and conductance switching of naphthalocyanine molecules, Science 317, 1203-1206 (2007)

24.54 P. Maksymovych, D.B. Dougherty, X.Y. Zhu, J.T. Yates Jr.: Nonlocal dissociative chemistry of adsorbed molecules induced by localized electron injection into metal surfaces, Phys. Rev. Lett. 99, 016101 (2007)

24.55 S. Katano, Y. Kim, M. Hori, M. Trenary, M. Kawai: Reversible control of hydrogenation of a single molecule, Science 316, 1883-1886 (2007)

24.56 G.V. Nazin, X.H. Qiu, W. Ho: Visualization and spectroscopy of a metal-molecule-metal bridge, Science 302, 77-81 (2003)

24.57 J. Repp, G. Meyer, S. Paavilainen, F.E. Olsson, $M$. Persson: Imaging bond formation between a gold atom and pentacene on an insulating surface, Science 312, 1196-1199 (2006)

24.58 R. Yamachika, M. Grobis, A. Wachowiak, M.F. Crommie: Controlled atomic doping of a single C-60 molecule, Science 304, 281-284 (2004)

24.59 S.W. Hla, L. Bartels, G. Meyer, K.H. Rieder: Inducing all steps of a chemical reaction with the scanning tunneling microscope tip: Towards single molecule engineering, Phys. Rev. Lett. 85, 2777-2780 (2000)
24.60 Y. Kim, T. Komeda, M. Kawai: Single-molecule reaction and characterization by vibrational excitation, Phys. Rev. Lett. 89, 126104 (2002)

24.61 M. Berthe, R. Stiufiuc, B. Grandidier, D. Deresmes, C. Delerue, D. Stievenard: Probing the carrier capture rate of a single quantum level, Science 319, 436-438 (2008)

24.62 N. Neel, J. Kroeger, L. Limot, K. Palotas, W.A. Hofer, R. Berndt: Conductance and Kondo effect in a controlled single-atom contact, Phys. Rev. Lett. 98, 016801 (2007)

24.63 J. Tersoff, D.R. Hamann: Theory and application for the scanning tunneling microscope, Phys. Rev. Lett. 50, 1998-2001 (1983)

24.64 C.J. Chen: Introduction to Scanning Tunneling Microscopy (0xford Univ. Press, 0xford 2007)

24.65 J. Wintterlin, J. Wiechers, H. Brune, T. Gritsch, H. Hofer, R.J. Behm: Atomic-resolution imaging of close-packed metal surfaces by scanning tunneling microscopy, Phys. Rev. Lett. 62, 59-62 (1989)

24.66 J.A. Stroscio, R.M. Feenstra, A.P. Fein: Electronic structure of the Si (111) $2 \times 1$ surface by scanningtunneling microscopy, Phys. Rev. Lett. 57, 25792582 (1986)

24.67 A.L.V. de Parga, 0.S. Hernan, R. Miranda, A.L. Yeyati, N. Mingo, A. Martin-Rodero, F. Flores: Electron resonances in sharp tips and their role in tunneling spectroscopy, Phys. Rev. Lett. 80, 357-360 (1998)

24.68 S.H. Pan, E.W. Hudson, J.C. Davis: Vacuum tunneling of superconducting quasiparticles from atomically sharp scanning tunneling microscope tips, Appl. Phys. Lett. 73, 2992-2994 (1998)

24.69 J.T. Li, W.D. Schneider, R. Berndt, O.R. Bryant, S. Crampin: Surface-state lifetime measured by scanning tunneling spectroscopy, Phys. Rev. Lett. 81, 4464-4467 (1998)

24.70 L. Burgi, 0. Jeandupeux, H. Brune, K. Kern: Probing hot-electron dynamics at surfaces with a cold scanning tunneling microscope, Phys. Rev. Lett. 82, 4516-4519 (1999)

24.71 J.W.G. Wildoer, C. Harmans, H. van Kempen: 0bservation of Landau levels at the InAs(110) surface by scanning tunneling spectroscopy, Phys. Rev. B 55, 16013-16016 (1997)

24.72 M. Morgenstern, V. Gudmundsson, R. Dombrowski, C. Wittneven, R. Wiesendanger: Nonlocality of the exchange interaction probed by scanning tunneling spectroscopy, Phys. Rev. B 201301, 63 (2001)

24.73 L. Limot, E. Pehlke, J. Kroger, R. Berndt: Surfacestate localization at adatoms, Phys. Rev. Lett. 94, 036805 (2005)

24.74 F.E. Olsson, M. Persson, A.G. Borisov, J.P. Gauyacq, J. Lagoute, S. Folsch: Localization of the Cu(111) surface state by single Cu adatoms, Phys. Rev. Lett. 93, 206803 (2004)

24.75 N. Nilius, T.M. Wallis, M. Persson, W. Ho: Distance dependence of the interaction between single atoms: Gold dimers on NiAl(110), Phys. Rev. Lett. 90, 196103 (2003) 
24.76 H.J. Lee, W. Ho, M. Persson: Spin splitting of s and $p$ states in single atoms and magnetic coupling in dimers on a surface, Phys. Rev. Lett. 92, 186802 (2004)

24.77 M.V. Grishin, F.I. Dalidchik, S.A. Kovalevskii, N.N. Kolchenko, B.R. Shub: Isotope effect in the vibrational spectra of water measured in experiments with a scanning tunneling microscope, Jetp Lett. 66, 37-40 (1997)

24.78 Y. Sainoo, Y. Kim, T. Okawa, T. Komeda, H. Shigekawa, M. Kawai: Excitation of molecular vibrational modes with inelastic scanning tunneling microscopy processes: Examination through action spectra of cis-2-butene on Pd(110), Phys. Rev. Lett. 95, 246102 (2005)

24.79 X.H. Qiu, G.V. Nazin, W. Ho: Vibronic states in single molecule electron transport, Phys. Rev. Lett. 92, 2016102 (2004)

24.80 A.C. Hewson: The Kondo Problem to Heavy Fermions (Cambridge Univ. Press, Cambridge 1993)

24.81 J.T. Li, W.D. Schneider, R. Berndt, B. Delley: Kondo scattering observed at a single magnetic impurity, Phys. Rev. Lett. 80, 2893-2896 (1998)

24.82 V. Madhavan, W. Chen, T. Jamneala, M.F. Crommie, N.S. Wingreen: Tunneling into a single magnetic atom: Spectroscopic evidence of the Kondo resonance, Science 280, 567-569 (1998)

24.83 T.W. Odom, J.L. Huang, C.L. Cheung, C.M. Lieber: Magnetic clusters on single-walled carbon nanotubes: The Kondo effect in a one-dimensional host, Science 290, 1549-1552 (2000)

24.84 M. Ouyang, J.L. Huang, C.L. Cheung, C.M. Lieber: Energy gaps in "metallic" single-walled carbon nanotubes, Science 292, 702-705 (2001)

24.85 U. Fano: Effects of configuration interaction on intensities and phase shifts, Phys. Rev. 124, 18661878 (1969)

24.86 P. Wahl, L. Diekhoner, M.A. Schneider, L. Vitali, G. Wittich, K. Kern: Kondo temperature of magnetic impurities at surfaces, Phys. Rev. Lett. 93, 176603 (2004)

24.87 Y.-S. Fu, S.-H. Ji, X. Chen, X.-C. Ma, R. Wu, C.C. Wang, W.-H. Duan, X.-H. Qiu, B. Sun, P. Zhang, J.-F. Jia, Q.-K. Xue: Manipulating the Kondo resonance through quantum size effects, Phys. Rev. Lett. 99, 256601 (2007)

24.88 J. Henzl, K. Morgenstern: Contribution of the surface state to the observation of the surface Kondo resonance, Phys. Rev. Lett. 98, 266601 (2007)

24.89 P. Wahl, P. Simon, L. Diekhoener, V.S. Stepanyuk, P. Bruno, M.A. Schneider, K. Kern: Exchange interaction between single magnetic adatoms, Phys. Rev. Lett. 98, 056601 (2007)

24.90 T. Jamneala, V. Madhavan, M.F. Crommie: Kondo response of a single antiferromagnetic chromium trimer, Phys. Rev. Lett. 87, 256804 (2001)

24.91 P. Wahl, L. Diekhoner, G. Wittich, L. Vitali, M.A. Schneider, K. Kern: Kondo effect of molecular complexes at surfaces: Ligand control of the local spin coupling, Phys. Rev. Lett. 95, 166601 (2005)

24.92 A.D. Zhao, Q.X. Li, L. Chen, H.J. Xiang, W.H. Wang, S. Pan, B. Wang, X.D. Xiao, J.L. Yang, J.G. Hou,
Q.S. Zhu: Controlling the Kondo effect of an adsorbed magnetic ion through its chemical bonding, Science 309, 1542-1544 (2005)

24.93 V. Iancu, A. Deshpande, S.-W. Hla: Manipulation of the Kondo effect via two-dimensional molecular assembly, Phys. Rev. Lett. 97, 266603 (2006)

24.94 L. Gao, W. Ji, Y.B. Hu, Z.H. Cheng, Z.T. Deng, Q. Liu, N. Jiang, X. Lin, W. Guo, S.X. Du, W.A. Hofer, X.C. Xie, H.J. Gao: Site-specific Kondo effect at ambient temperatures in iron-based molecules, Phys. Rev. Lett. 99, 106402 (2007)

24.95 H.C. Manoharan, C.P. Lutz, D.M. Eigler: Quantum mirages formed by coherent projection of electronic structure, Nature 403, 512-515 (2000)

24.96 H.A. Mizes, J.S. Foster: Long-range electronic perturbations caused by defects using scanning tunneling microscopy, Science 244, 559-562 (1989)

24.97 P.T. Sprunger, L. Petersen, E.W. Plummer, E. Laegsgaard, F. Besenbacher: Giant friedel oscillations on the beryllium(0001) surface, Science $275,1764-$ 1767 (1997)

24.98 P. Hofmann, B.G. Briner, M. Doering, H.P. Rust, E.W. Plummer, A.M. Bradshaw: Anisotropic twodimensional Friedel oscillations, Phys. Rev. Lett. 79, 265-268 (1997)

24.99 E.J. Heller, M.F. Crommie, C.P. Lutz, D.M. Eigler: Scattering and absorption of surface electron waves in quantum corrals, Nature 369, 464-466 (1994)

24.100 M. van der Wielen, A.J.A. van Roij, H. van Kempen: Direct observation of Friedel oscillations around incorporated sie, dopants in GaAs by low-temperature scanning tunneling microscopy, Phys. Rev. Lett. 76, 1075-1078 (1996)

24.101 T. Maltezopoulos, A. Bolz, C. Meyer, C. Heyn, W. Hansen, M. Morgenstern, R. Wiesendanger: Wave-function mapping of InAs quantum dots by scanning tunneling spectroscopy, Phys. Rev. Lett. 91, 196804 (2003)

24.102 0. Millo, D. Katz, Y.W. Cao, U. Banin: Imaging and spectroscopy of artificial-atom states in core/shell nanocrystal quantum dots, Phys. Rev. Lett. 86, 5751-5754 (2001)

24.103 R. Temirov, S. Soubatch, A. Luican, F.S. Tautz: Freeelectron-like dispersion in an organic monolayer film on a metal substrate, Nature 444, 350-353 (2006)

24.104 K. Suzuki, K. Kanisawa, C. Janer, S. Perraud, K. Takashina, T. Fujisawa, Y. Hirayama: Spatial imaging of two-dimensional electronic states in semiconductor quantum wells, Phys. Rev. Lett. 98, 136802 (2007)

24.105 S.G. Lemay, J.W. Janssen, M. van den Hout, M. Mooij, M.J. Bronikowski, P.A. Willis, R.E. Smalley, L.P. Kouwenhoven, C. Dekker: Two-dimensional imaging of electronic wavefunctions in carbon nanotubes, Nature 412, 617-620 (2001)

24.106 L.C. Venema, J.W.G. Wildoer, J.W. Janssen, S.J. Tans, H. Tuinstra, L.P. Kouwenhoven, C. Dekker: Imaging electron wave functions of quantized energy levels in carbon nanotubes, Science 283, 52-55 (1999) 
24.107 C.R. Moon, L.S. Mattos, B.K. Foster, G. Zeltzer, W. Ko, H.C. Manoharan: Quantum phase extraction in isospectral electronic nanostructures, Science 319, 782-787 (2008)

24.108 X.H. Lu, M. Grobis, K.H. Khoo, S.G. Louie, M.F. Crommie: Spatially mapping the spectral density of a single (-60 molecule, Phys. Rev. Lett. 90, 096802 (2003)

24.109 F. Marczinowski, J. Wiebe, J.M. Tang, M.E. Flatte, F. Meier, M. Morgenstern, R. Wiesendanger: Local electronic structure near $\mathrm{mn}$ acceptors in InAs: Surface-induced symmetry breaking and coupling to host states, Phys. Rev. Lett. 99, 157202 (2007)

24.110 A.M. Yakunin, A.Y. Silov, P.M. Koenraad, J.H. Wolter, W. Van Roy, J. De Boeck, J.M. Tang, M.E. Flatte: Spatial structure of an individual Mn acceptor in GaAs, Phys. Rev. Lett. 92, 216806 (2004)

24.111 J.N. Crain, D.T. Pierce: End states in one-dimensional atom chains, Science 307, 703-706 (2005)

24.112 N. Nilius, T.M. Wallis, W. Ho: Development of one-dimensional band structure in artificial gold chains, Science 297, 1853-1856 (2002)

24.113 D. Kitchen, A. Richardella, J.-M. Tang, M.E. Flatte, A. Yazdani: Atom-by-atom substitution of $\mathrm{Mn}$ in GaAs and visualization of their hole-mediated interactions, Nature 442, 436-439 (2006)

24.114 C. Wittneven, R. Dombrowski, M. Morgenstern, R. Wiesendanger: Scattering states of ionized dopants probed by low temperature scanning tunneling spectroscopy, Phys. Rev. Lett. 81, 56165619 (1998)

24.115 D. Haude, M. Morgenstern, I. Meinel, R. Wiesendanger: Local density of states of a three-dimensional conductor in the extreme quantum limit, Phys. Rev. Lett. 86, 1582-1585 (2001)

24.116 R. Joynt, R.E. Prange: Conditions for the quantum hall effect, Phys. Rev. B 29, 3303-3317 (1984)

24.117 M. Morgenstern, J. Klijn, C. Meyer, R. Wiesendanger: Real-space observation of drift states in a two-dimensional electron system at high magnetic fields, Phys. Rev. Lett. 90, 056804 (2003)

24.118 M. Morgenstern, J. Klijn, C. Meyer, M. Getzlaff, R. Adelung, R.A. Romer, K. Rossnagel, L. Kipp, M. Skibowski, R. Wiesendanger: Direct comparison between potential landscape and local density of states in a disordered two-dimensional electron system, Phys. Rev. Lett. 89, 136806 (2002)

24.119 E. Abrahams, P.W. Anderson, D.C. Licciardello, T.V. Ramakrishnan: Scaling theory of localization: Absence of quantum diffusion in two dimensions, Phys. Rev. 42, 673-676 (1979)

24.120 C. Meyer, J. Klijn, M. Morgenstern, R. Wiesendanger: Direct measurement of the local density of states of a disordered one-dimensional conductor, Phys. Rev. Lett. 91, 076803 (2003)

24.121 N. Oncel, A. van Houselt, J. Huijben, A.S. Hallback, 0. Gurlu, H.J.W. Zandvliet, B. Poelsema: Quantum confinement between self-organized Pt nanowires on Ge(001), Phys. Rev. Lett. 95, 116801 (2005)
24.122 J. Lee, S. Eggert, H. Kim, S.J. Kahng, H. Shinohara, Y. Kuk: Real space imaging of one-dimensional standing waves: Direct evidence for a Luttinger liquid, Phys. Rev. Lett. 93, 166403 (2004)

24.123 R.E. Peierls: Quantum Theory of Solids (Clarendon, Oxford 1955)

24.124 C.G. Slough, W.W. McNairy, R.V. Coleman, B. Drake, P.K. Hansma: Charge-density waves studied with the use of a scanning tunneling microscope, Phys. Rev. B 34, 994-1005 (1986)

24.125 X.L. Wu, C.M. Lieber: Hexagonal domain-like charge density wave phase of $\mathrm{TaS}_{2}$ determined by scanning tunneling microscopy, Science 243, 1703-1705 (1989)

24.126 T. Nishiguchi, M. Kageshima, N. Ara-Kato, A. Kawazu: Behavior of charge density waves in a one-dimensional organic conductor visualized by scanning tunneling microscopy, Phys. Rev. Lett. 81, 3187-3190 (1998)

24.127 X.L. Wu, C.M. Lieber: Direct observation of growth and melting of the hexagonal-domain chargedensity-wave phase in $1 \mathrm{~T}-\mathrm{TaS}_{2}$ by scanning tunneling microscopy, Phys. Rev. Lett. 64, 1150-1153 (1990)

24.128 J.M. Carpinelli, H.H. Weitering, E.W. Plummer, R. Stumpf: Direct observation of a surface charge density wave, Nature 381, 398-400 (1996)

24.129 H.H. Weitering, J.M. Carpinelli, A.P. Melechko, J.D. Zhang, M. Bartkowiak, E.W. Plummer: Defectmediated condensation of a charge density wave, Science 285, 2107-2110 (1999)

24.130 S. Modesti, L. Petaccia, G. Ceballos, I. Vobornik, G. Panaccione, G. Rossi, L. Ottaviano, R. Larciprete, S. Lizzit, A. Goldoni: Insulating ground state of Sn/Si(111)-(root $3 \times$ root 3)R30 degrees, Phys. Rev. Lett. 98, 126401 (2007)

24.131 K. Swamy, A. Menzel, R. Beer, E. Bertel: Charge-density waves in self-assembled halogen-bridged metal chains, Phys. Rev. Lett. 86, 1299-1302 (2001)

24.132 H.W. Yeom, S. Takeda, E. Rotenberg, I. Matsuda, K. Horikoshi, J. Schaefer, C.M. Lee, S.D. Kevan, T. Ohta, T. Nagao, S. Hasegawa: Instability and charge density wave of metallic quantum chains on a silicon surface, Phys. Rev. Lett. 82, 48984901 (1999)

24.133 J.J. Kim, W. Yamaguchi, T. Hasegawa, K. Kitazawa: Observation of mott localization gap using low temperature scanning tunneling spectroscopy in commensurate $1 \mathrm{~T}-\mathrm{T}$ a Sa 2, Phys. Rev. Lett. 73, 2103-2106 (1994)

24.134 A. Wachowiak, R. Yamachika, K.H. Khoo, Y. Wang, M. Grobis, D.H. Lee, S.G. Louie, M.F. Crommie: Visualization of the molecular Jahn-Teller effect in an insulating $\mathrm{K}_{4} \mathrm{C}_{60}$ monolayer, Science 310 , 468470 (2005)

24.135 J. Bardeen, L.N. Cooper, J.R. Schrieffer: Theory of superconductivity, Phys. Rev. 108, 1175-1204 (1957)

24.136 A. Yazdani, B.A. Jones, C.P. Lutz, M.F. Crommie, D.M. Eigler: Probing the local effects of magnetic impurities on superconductivity, Science 275, 1767-1770 (1997) 
24.137 K. Inoue, H. Takayanagi: Local tunneling spectroscopy of a Nb/InAs/Nb superconducting proximity system with a scanning tunneling microscope, Phys. Rev. B 43, 6214-6215 (1991)

24.138 S.H. Tessmer, M.B. Tarlie, D.J. VanHarlingen, D.L. Maslov, P.M. Goldbart: Probing the superconducting proximity effect in $\mathrm{NbSe}_{2}$ by scanning tunneling microscopy, Phys. Rev. Lett. 77, 924-927 (1996)

24.139 H.F. Hess, R.B. Robinson, R.C. Dynes, J.M. Valles, J.V. Waszczak: Scanning-tunneling-microscope observation of the Abrikosov flux lattice and the density of states near and inside a fluxoid, Phys. Rev. Lett. 62, 214-216 (1989)

24.140 H.F. Hess, R.B. Robinson, J.V. Waszczak: Vortexcore structure observed with a scanning tunneling microscope, Phys. Rev. Lett. 64, 2711-2714 (1990)

24.141 N. Hayashi, M. Ichioka, K. Machida: Star-shaped local density of states around vortices in a typeII superconductor, Phys. Rev. Lett. 77, 4074-4077 (1996)

24.142 H. Sakata, M. Oosawa, K. Matsuba, N. Nishida, H. Takeya, K. Hirata: Imaging of a vortex lattice transition in $\mathrm{YNi}_{2} \mathrm{~B}_{2} \mathrm{C}$ by scanning tunneling spectroscopy, Phys. Rev. Lett. 84, 1583-1586 (2000)

24.143 S. Behler, S.H. Pan, P. Jess, A. Baratoff, H.J. Guntherodt, F. Levy, G. Wirth, J. Wiesner: Vortex pinning in ion-irradiated $\mathrm{NbSe}_{2}$ studied by scanning tunneling microscopy, Phys. Rev. Lett. 72, 17501753 (1994)

24.144 R. Berthe, U. Hartmann, C. Heiden: Influence of a transport current on the Abrikosov flux lattice observed with a low-temperature scanning tunneling microscope, Ultramicroscopy 42, 696-698 (1992)

24.145 N.C. Yeh, C.T. Chen, G. Hammerl, J. Mannhart, A. Schmehl, C.W. Schneider, R.R. Schulz, S. Tajima, K. Yoshida, D. Garrigus, M. Strasik: Evidence of doping-dependent pairing symmetry in cuprate superconductors, Phys. Rev. Lett. 87, 087003 (2001)

24.146 K. McElroy, R.W. Simmonds, J.E. Hoffman, D.H. Lee, J. Orenstein, H. Eisaki, S. Uchida, J.C. Davis: Relating atomic-scale electronic phenomena to wave-like quasiparticle states in superconducting $\mathrm{Bi}_{2} \mathrm{Sr}_{2} \mathrm{CaCu}_{2} \mathrm{O}_{8}+\delta$, Nature 422, 592-596 (2003)

24.147 K. McElroy, J. Lee, J.A. Slezak, D.H. Lee, H. Eisaki, S. Uchida, J.C. Davis: Atomic-scale sources and mechanism of nanoscale electronic disorder in $\mathrm{Bi}_{2} \mathrm{Sr}_{2} \mathrm{CaCu}_{2} \mathrm{O}_{8+\delta}$, Science 309, 1048-1052 (2005)

24.148 S.H. Pan, E.W. Hudson, K.M. Lang, H. Eisaki, S. Uchida, J.C. Davis: Imaging the effects of individual zinc impurity atoms on superconductivity in $\mathrm{Bi}_{2} \mathrm{Sr}_{2} \mathrm{CaCu}_{2} \mathrm{O}_{8+\delta}$, Nature 403, 746-750 (2000)

24.149 A. Polkovnikov, S. Sachdev, M. Vojta: Impurity in a d-wave superconductor: Kondo effect and STM spectra, Phys. Rev. Lett. 86, 296-299 (2001)

24.150 A.N. Pasupathy, A. Pushp, K.K. Gomes, C.V. Parker, J. Wen, Z. Xu, G. Gu, S. Ono, Y. Ando, A. Yazdani: Electronic origin of the inhomogeneous pairing interaction in the high-T-c superconductor $\mathrm{Bi}_{2} \mathrm{Sr}_{2} \mathrm{CaCu}_{2} \mathrm{O}_{8+\delta}$, Science 320, 196-201 (2008)

24.151 I. Maggioaprile, C. Renner, A. Erb, E. Walker, 0 . Fischer: Direct vortex lattice imaging and tunneling spectroscopy of flux lines on $\mathrm{YBa}_{2} \mathrm{Cu}_{3} \mathrm{O}_{7-\delta}$, Phys. Rev. Lett. 75, 2754-2757 (1995)

24.152 S.H. Pan, E.W. Hudson, A.K. Gupta, K.W. Ng, H. Eisaki, S. Uchida, J.C. Davis: STM studies of the electronic structure of vortex cores in $\mathrm{Bi}_{2} \mathrm{Sr}_{2} \mathrm{CaCu}_{2} \mathrm{O}_{8+\delta}$, Phys. Rev. Lett. 85, 1536-1539 (2000)

24.153 C. Renner, B. Revaz, K. Kadowaki, I. Maggio-Aprile, 0. Fischer: Observation of the low temperature pseudogap in the vortex cores of $\mathrm{Bi}_{2} \mathrm{Sr}_{2} \mathrm{CaCu}_{2} \mathrm{O}_{8+\delta}$, Phys. Rev. Lett. 80, 3606-3609 (1998)

24.154 D.P. Arovas, A.J. Berlinsky, C. Kallin, S.C. Zhang: Superconducting vortex with antiferromagnetic core, Phys. Rev. Lett. 79, 2871-2874 (1997)

24.155 J.E. Hoffman, E.W. Hudson, K.M. Lang, V. Madhavan, H. Eisaki, S. Uchida, J.C. Davis: A four unit cell periodic pattern of quasi-particle states surrounding vortex cores in $\mathrm{Bi}_{2} \mathrm{Sr}_{2} \mathrm{CaCu}_{2} \mathrm{O}_{8+\delta}$, Science 295, 466-469 (2002)

24.156 M. Vershinin, S. Misra, S. Ono, Y. Abe, Y. Ando, A. Yazdani: Local ordering in the pseudogap state of the high-T-c superconductor $\mathrm{Bi}_{2} \mathrm{Sr}_{2} \mathrm{CaCu}_{2} \mathrm{O}_{8}+\delta$, Science 303, 1995-1998 (2004)

24.157 T. Hanaguri, C. Lupien, Y. Kohsaka, D.H. Lee, M. Azuma, M. Takano, H. Takagi, J.C. Davis: A 'checkerboard' electronic crystal state in lightly hole-doped $\mathrm{Ca}_{2-x} \mathrm{Na}_{x} \mathrm{CuO}_{2} \mathrm{Cl}_{2}$, Nature 430, 10011005 (2004)

24.158 Y. Kohsaka, C. Taylor, K. Fujita, A. Schmidt, C. Lupien, T. Hanaguri, M. Azuma, M. Takano, H. Eisaki, H. Takagi, S. Uchida, J.C. Davis: An intrinsic bond-centered electronic glass with unidirectional domains in underdoped cuprates, Science 315, 1380-1385 (2007)

24.159 0. Naaman, W. Teizer, R.C. Dynes: Fluctuation dominated Josephson tunneling with a scanning tunneling microscope, Phys. Rev. Lett. 87, 097004 (2001)

24.160 M. Bode, M. Getzlaff, R. Wiesendanger: Spinpolarized vacuum tunneling into the exchangesplit surface state of $\mathrm{Gd}(0001)$, Phys. Rev. Lett. 81, 4256-4259 (1998)

24.161 A. Kubetzka, M. Bode, 0. Pietzsch, R. Wiesendanger: Spin-polarized scanning tunneling microscopy with antiferromagnetic probe tips, Phys. Rev. Lett. 88, 057201 (2002)

24.162 0. Pietzsch, A. Kubetzka, M. Bode, R. Wiesendanger: Observation of magnetic hysteresis at the nanometer scale by spin-polarized scanning tunneling spectroscopy, Science 292, 2053-2056 (2001)

24.163 S. Heinze, M. Bode, A. Kubetzka, 0. Pietzsch, $X$. Nie, S. Blugel, R. Wiesendanger: Real-space imaging of two-dimensional antiferromagnetism on the atomic scale, Science 288, 1805-1808 (2000) 
24.164 A. Kubetzka, P. Ferriani, M. Bode, S. Heinze, G. Bihlmayer, K. von Bergmann, 0. Pietzsch, S. Blugel, R. Wiesendanger: Revealing antiferromagnetic order of the Fe monolayer on W(001): Spin-polarized scanning tunneling microscopy and first-principles calculations, Phys. Rev. Lett. 94, 087204 (2005)

24.165 A. Wachowiak, J. Wiebe, M. Bode, 0. Pietzsch, M. Morgenstern, R. Wiesendanger: Direct observation of internal spin structure of magnetic vortex cores, Science 298, 577-580 (2002)

24.166 M. Bode, M. Heide, K. von Bergmann, P. Ferriani, S. Heinze, G. Bihlmayer, A. Kubetzka, 0. Pietzsch, S. Bluegel, R. Wiesendanger: Chiral magnetic order at surfaces driven by inversion asymmetry, Nature 447, 190-193 (2007)

24.167 C.L. Gao, U. Schlickum, W. Wulfhekel, J. Kirschner: Mapping the surface spin structure of large unit cells: Reconstructed Mn films on Fe(001), Phys. Rev. Lett. 98, 107203 (2007)

24.168 K. Von Bergmann, S. Heinze, M. Bode, E.Y. Vedmedenko, G. Bihlmayer, S. Blugel, R. Wiesendanger: Observation of a complex nanoscale magnetic structure in a hexagonal Fe monolayer, Phys. Rev. Lett. 96, 167203 (2006)

24.169 Y. Yayon, V.W. Brar, L. Senapati, S.C. Erwin, M.F. Crommie: Observing spin polarization of individual magnetic adatoms, Phys. Rev. Lett. 99, 067202 (2007)

24.170 M. Bode, 0. Pietzsch, A. Kubetzka, R. Wiesendanger: Shape-dependent thermal switching behavior of superparamagnetic nanoislands, Phys. Rev. Lett. 92, 067201 (2004)

24.171 S. Krause, L. Berbil-Bautista, G. Herzog, M. Bode, R. Wiesendanger: Current-induced magnetization switching with a spin-polarized scanning tunneling microscope, Science 317, 1537-1540 (2007)

24.172 P.J. Eaton, P. West: Atomic Force Microscopy (0xford Univ. Press, Oxford 2010)

24.173 R. Garcia, R. Perez: Dynamic atomic force microscopy methods, Surf. Sci. Rep. 47, 197-301 (2002)

24.174 U. Dürig: Extracting interaction forces and complementary observables in dynamic probe microscopy, Appl. Phys. Lett. 76, 1203-1205 (2000)

24.175 F.J. Giessibl: A direct method to calculate tipsample forces from frequency shifts in frequencymodulation atomic force microscopy, Appl. Phys. Lett. 78, 123-125 (2001)

24.176 B. Gotsmann, B. Anczykowski, C. Seidel, H. Fuchs: Determination of tip-sample interaction forces from measured dynamic force spectroscopy curves, Appl. Surf. Sci. 140, 314-319 (1999)

24.177 J.E. Sader, S.P. Jarvis: Accurate formulas for interaction force and energy in frequency modulation force spectroscopy, Appl. Phys. Lett. 84, 1801-1803 (2004)

24.178 F.J. Giessibl: Atomic resolution of the silicon (111)$(7 \times 7)$ surface by atomic force microscopy, Science 267, 68-71 (1995)
24.179 M.A. Lantz, H.J. Hug, P.J.A. van Schendel, R. Hoffmann, S. Martin, A. Baratoff, A. Abdurixit, H.J. Guntherodt, C. Gerber: Low temperature scanning force microscopy of the $\mathrm{Si}(111)-(7 \times 7)$ surface, Phys. Rev. Lett. 84, 2642-2645 (2000)

24.180 N. Suehira, Y. Sugawara, S. Morita: Artifact and fact of Si(111)7 77 surface images observed with a low temperature noncontact atomic force microscope (LT-NC-AFM), Jpn. J. Appl. Phys. Part 2 Lett. 40, L292-L294 (2001)

24.181 K. Suzuki, M. Iwatsuki, S. Kitamura, C.B. Mooney: Development of low temperature ultrahigh vacuum atomic force microscope/scanning tunneling microscope, Jpn. J. Appl. Phys. Part 1 Regul. Pap. Short Notes Rev. Pap. 39, 3750-3752 (2000)

24.182 T. Uozumi, Y. Tomiyoshi, N. Suehira, Y. Sugawara, S. Morita: Observation of Si(100) surface with noncontact atomic force microscope at $5 \mathrm{~K}$, Appl. Surf. Sci. 188, 279-284 (2002)

24.183 Y.J. Li, H. Nomura, N. Ozaki, Y. Naitoh, M. Kageshima, Y. Sugawara, C. Hobbs, L. Kantorovich: Origin of $\mathrm{p}(2 \times 1)$ phase on $\mathrm{Si}(001)$ by noncontact atomic force microscopy at $5 \mathrm{~K}$, Phys. Rev. Lett. 96, 106104 (2006)

24.184 A. Sweetman, S. Jarvis, R. Danza, J. Bamidele, S. Gangopadhyay, G.A. Shaw, L. Kantorovich, P. Moriarty: Toggling bistable atoms via mechanical switching of bond angle, Phys. Rev. Lett. 106, 136101 (2011)

24.185 A. Sweetman, S. Jarvis, R. Danza, J. Bamidele, L. Kantorovich, P. Moriarty: Manipulating Si(100) at $5 \mathrm{~K}$ using qPlus frequency modulated atomic force microscopy: Role of defects and dynamics in the mechanical switching of atoms, Phys. Rev. B 84, 085426 (2011)

24.186 A. Schwarz, W. Allers, U.D. Schwarz, R. Wiesendanger: Simultaneous imaging of the in and as sublattice on $\operatorname{InAs}(110)-(1 \times 1)$ with dynamic scanning force microscopy, Appl. Surf. Sci. 140, 293-297 (1999)

24.187 A. Schwarz, W. Allers, U.D. Schwarz, R. Wiesendanger: Dynamic-mode scanning force $\mathrm{mi}-$ croscopy study of $n-\ln A s(110)-(1 \times 1)$ at low temperatures, Phys. Rev. B 61, 2837-2845 (2000)

24.188 A. Sweetman, A. Stannard, Y. Sugimoto, M. Abe, S. Morita, P. Moriarty: Simultaneous noncontact AFM and STM of Ag:Si(111) $-(\sqrt{3} \times \sqrt{3})$ R30 $0^{\circ}$, Phys. Rev. B 87, 075310 (2013)

24.189 W. Allers, A. Schwarz, U.D. Schwarz, R. Wiesendanger: Dynamic scanning force microscopy at low temperatures on a van der Waals surface: graphite (0001), Appl. Surf. Sci. 140, 247-252 (1999)

24.190 M. Ashino, A. Schwarz, H. Holscher, U.D. Schwarz, R. Wiesendanger: Interpretation of the atomic scale contrast obtained on graphite and singlewalled carbon nanotubes in the dynamic mode of atomic force microscopy, Nanotechnology 16, S134-S137 (2005)

24.191 S. Hembacher, F.J. Giessibl, J. Mannhart, C.F. Quate: Local spectroscopy and atomic imaging of tunneling current, forces, and dissipation on graphite, Phys. Rev. Lett. 94, 056101 
(2005)

24.192 H. Holscher, W. Allers, U.D. Schwarz, A. Schwarz, R. Wiesendanger: Interpretation of "true atomic resolution" images of graphite (0001) in noncontact atomic force microscopy, Phys. Rev. B 62, 6967-6970 (2000)

24.193 W. Allers, A. Schwarz, U.D. Schwarz, R. Wiesendanger: Dynamic scanning force microscopy at low temperatures on a noble-gas crystal: Atomic resolution on the xenon(111) surface, Europhys. Lett. 48, 276-279 (1999)

24.194 M.Z. Baykara, H. Moenig, T.C. Schwendemann, 0. Uenverdi, E.I. Altman, U.D. Schwarz: Threedimensional interaction force and tunneling current spectroscopy of point defects on rutile $\mathrm{TiO}_{2}$ (110), Appl. Phys. Lett. 108, 071601 (2016)

24.195 A. Yurtsever, Y. Sugimoto, M. Abe, S. Morita: NCAFM imaging of the TiO(2)(110)-(1 $\times 1)$ surface at low temperature, Nanotechnology 21, 165702 (2010)

24.196 R. Hoffmann, M.A. Lantz, H.J. Hug, P.J.A. van Schendel, P. Kappenberger, S. Martin, A. Baratoff, H.J. Guntherodt: Atomic resolution imaging and force versus distance measurements on $\mathrm{KBr}(001)$ using low temperature scanning force microscopy, Appl. Surf. Sci. 188, 238-244 (2002)

24.197 D.G. de Oteyza, P. Gorman, Y.-C. Chen, S. Wickenburg, A. Riss, D.J. Mowbray, G. Etkin, Z. Pedramrazi, H.-Z. Tsai, A. Rubio, M.F. Crommie, F.R. Fischer: Direct imaging of covalent bond structure in single-molecule chemical reactions, Science 340, 1434-1437 (2013)

24.198 L. Gross: Recent advances in submolecular resolution with scanning probe microscopy, Nat. Chem. 3, 273-278 (2011)

24.199 L. Gross, F. Mohn, N. Moll, P. Liljeroth, G. Meyer: The chemical structure of a molecule resolved by atomic force microscopy, Science 325, 1110-1114 (2009)

24.200 L. Gross, F. Mohn, N. Moll, G. Meyer, R. Ebel, W.M. Abdel-Mageed, M. Jaspars: Organic structure determination using atomic-resolution scanning probe microscopy, Nat. Chem. 2, 821-825 (2010)

24.201 L. Gross, F. Mohn, N. Moll, B. Schuler, A. Criado, E. Guitian, D. Pena, A. Gourdon, G. Meyer: Bondorder discrimination by atomic force microscopy, Science 337, 1326-1329 (2012)

24.202 B. Schuler, G. Meyer, D. Pena, 0.C. Mullins, L. Gross: Unraveling the molecular structures of asphaltenes by atomic force microscopy, J. Am. Chem. Soc. 137, 9870-9876 (2015)

24.203 A.M. Sweetman, S.P. Jarvis, H.Q. Sang, I. Lekkas, P. Rahe, Y. Wang, J.B. Wang, N.R. Champness, L. Kantorovich, P. Moriarty: Mapping the force field of a hydrogen-bonded assembly, Nat. Commun. 5, 3931 (2014)

24.204 P. Hapala, G. Kichin, C. Wagner, F.S. Tautz, R. Temirov, P. Jelinek: Mechanism of high-resolution STM/AFM imaging with functionalized tips, Phys. Rev. B 90, 085421 (2014)

24.205 N. Moll, L. Gross, F. Mohn, A. Curioni, G. Meyer: The mechanisms underlying the enhanced resolution of atomic force microscopy with functionalized tips, New J. Phys. 12, 125020 (2010)

24.206 F. Mohn, L. Gross, N. Moll, G. Meyer: Imaging the charge distribution within a single molecule, Nat. Nanotechnol. 7, 227-231 (2012)

24.207 B. Schuler, S.-X. Liu, Y. Geng, S. Decurtins, G. Meyer, L. Gross: Contrast formation in Kelvin probe force microscopy of single pi-conjugated molecules, Nano Lett. 14, 3342-3346 (2014)

24.208 H. Holscher, W. Allers, U.D. Schwarz, A. Schwarz, R. Wiesendanger: Determination of tip-sample interaction potentials by dynamic force spectroscopy, Phys. Rev. Lett. 83, 4780-4783 (1999)

24.209 H. Holscher, U.D. Schwarz, R. Wiesendanger: Calculation of the frequency shift in dynamic force microscopy, Appl. Surf. Sci. 140, 344-351 (1999)

24.210 R. Hoffmann, C. Barth, A.S. Foster, A.L. Shluger, H.J. Hug, H.J. Guntherodt, R.M. Nieminen, M. Reichling: Measuring site-specific cluster-surface bond formation, J. Am. Chem. Soc. 127, 1786317866 (2005)

24.211 H. Holscher, A. Schwarz, W. Allers, U.D. Schwarz, R. Wiesendanger: Quantitative analysis of dynamic-force-spectroscopy data on graphite(0001) in the contact and noncontact regimes, Phys. Rev. B 61, 12678-12681 (2000)

24.212 M.A. Lantz, R. Hoffmann, A.S. Foster, A. Baratoff, H.J. Hug, H.R. Hidber, H.J. Guntherodt: Site-specific force-distance characteristics on $\mathrm{NaCl}(001)$ : Measurements versus atomistic simulations, Phys. Rev. B 74, 245426 (2006)

24.213 M.A. Lantz, H.J. Hug, R. Hoffmann, P.J.A. van Schendel, P. Kappenberger, S. Martin, A. Baratoff, H.J. Guntherodt: Quantitative measurement of short-range chemical bonding forces, Science 291, 2580-2583 (2001)

24.214 Z.X. Sun, M.P. Boneschanscher, I. Swart, D. Vanmaekelbergh, P. Liljeroth: Quantitative atomic force microscopy with carbon monoxide terminated tips, Phys. Rev. Lett. 106, 046104 (2011)

24.215 Y. Sugimoto, P. Pou, M. Abe, P. Jelinek, R. Perez, S. Morita, 0. Custance: Chemical identification of individual surface atoms by atomic force microscopy, Nature 446, 64-67 (2007)

24.216 M. Abe, Y. Sugimoto, 0. Custance, S. Morita: Atom tracking for reproducible force spectroscopy at room temperature with non-contact atomic force microscopy, Nanotechnology 16, 3029-3034 (2005)

24.217 M.Z. Baykara, M. Todorovic, H. Monig, T.C. Schwendemann, 0. Unverdi, L. Rodrigo, E.I. Altman, R. Perez, U.D. Schwarz: Atom-specific forces and defect identification on surfaceoxidized $\mathrm{Cu}(100)$ with combined 3D-AFM and STM measurements, Phys. Rev. B 87, 155414 (2013)

24.218 H. Holscher, S.M. Langkat, A. Schwarz, R. Wiesendanger: Measurement of three-dimensional force fields with atomic resolution using dynamic force spectroscopy, Appl. Phys. Lett. 81, 4428-4430 (2002)

24.219 M.Z. Baykara, T.C. Schwendemann, B.J. Albers, N. Pilet, H. Monig, E.I. Altman, U.D. Schwarz: Exploring atomic-scale lateral forces in the at- 
tractive regime: A case study on graphite (0001), Nanotechnology 23, 405703 (2012)

24.220 M.Z. Baykara, T.C. Schwendemann, E.I. Altman, U.D. Schwarz: Three-dimensional atomic force microscopy-taking surface imaging to the next level, Adv. Mater. 22, 2838-2853 (2010)

24.221 B.J. Albers, T.C. Schwendemann, M.Z. Baykara, N. Pilet, M. Liebmann, E.I. Altman, U.D. Schwarz: Data acquisition and analysis procedures for high-resolution atomic force microscopy in three dimensions, Nanotechnology 20, 264002 (2009)

24.222 M.Z. Baykara, O.E. Dagdeviren, T.C. Schwendemann, H. Monig, E.I. Altman, U.D. Schwarz: Probing three-dimensional surface force fields with atomic resolution: Measurement strategies, limitations and artifact reduction, Beilstein J. Nanotechnol. 3, 637-650 (2012)

24.223 S. Kawai, T. Glatzel, S. Koch, A. Baratoff, E. Meyer: Interaction-induced atomic displacements revealed by drift-corrected dynamic force spectroscopy, Phys. Rev. B 83, 035421 (2011)

24.224 Y. Sugimoto, K. Ueda, M. Abe, S. Morita: Three-dimensional scanning force/tunneling spectroscopy at room temperature, J. Phys. Condens. Matter 24, 084008 (2012)

24.225 S. Fremy, S. Kawai, R. Pawlak, T. Glatzel, A. Baratoff, E. Meyer: Three-dimensional dynamic force spectroscopy measurements on $\operatorname{KBr}(001)$ : Atomic deformations at small tip-sample separations, Nanotechnology 23, 055401 (2012)

24.226 R. Pawlak, S. Kawai, S. Fremy, T. Glatzel, E. Meyer: Atomic-scale mechanical properties of orientated C(60) molecules revealed by noncontact atomic force microscopy, ACS Nano 5, 6349-6354 (2011)

24.227 N. Oyabu, 0. Custance, I.S. Yi, Y. Sugawara, S. Morita: Mechanical vertical manipulation of selected single atoms by soft nanoindentation using near contact atomic force microscopy, Phys. Rev. Lett. 90, 176102 (2003)

24.228 N. Oyabu, Y. Sugimoto, M. Abe, 0. Custance, S. Morita: Lateral manipulation of single atoms at semiconductor surfaces using atomic force microscopy, Nanotechnology 16, S112-S117 (2005)

24.229 J. Bamidele, S.H. Lee, Y. Kinoshita, R. Turansky, Y. Naitoh, Y.J. Li, Y. Sugawara, I. Stich, L. Kantorovich: Vertical atomic manipulation with dynamic atomic-force microscopy without tip change via a multi-step mechanism, Nat. Commun. 5, 5476 (2014)

24.230 Y. Sugimoto, M. Abe, S. Hirayama, N. Oyabu, 0 . Custance, S. Morita: Atom inlays performed at room temperature using atomic force microscopy, Nat. Mater. 4, 156-159 (2005)

24.231 Y. Sugimoto, P. Pou, 0. Custance, P. Jelinek, M. Abe, R. Perez, S. Morita: Complex patterning by vertical interchange atom manipulation using atomic force microscopy, Science 322, 413-417 (2008)

24.232 S. Kawai, A.S. Foster, F.F. Canova, H. Onodera, S.-I. Kitamura, E. Meyer: Atom manipulation on an insulating surface at room temperature, Nat. Commun. 5, 5403 (2014)
24.233 S. Hirth, F. Ostendorf, M. Reichling: Lateral manipulation of atomic size defects on the $\mathrm{CaF}_{2}(111)$ surface, Nanotechnology 17, S148-S154 (2006)

24.234 S. Kawai, M. Koch, E. Gnecco, A. Sadeghi, R. Pawlak, T. Glatzel, J. Schwarz, S. Goedecker, S. Hecht, A. Baratoff, L. Grill, E. Meyer: Quantifying the atomic-level mechanics of single long physisorbed molecular chains, Proc. Natl. Acad. Sci. U.S.A. 111, 3968-3972 (2014)

24.235 G. Langewisch, J. Falter, H. Fuchs, A. Schirmeisen: Forces during the controlled displacement of organic molecules, Phys. Rev. Lett. 110, 036101 (2013)

24.236 R. Pawlak, W. Ouyang, A.E. Filippov, L. Kalikhman-Razvozov, S. Kawai, T. Glatzel, E. Gnecco, A. Baratoff, Q. Zheng, 0. Hod, M. Urbakh, E. Meyer: Single-molecule tribology: Force microscopy manipulation of a porphyrin derivative on a copper surface, ACS Nano 10, 713-722 (2016)

24.237 R. Pawlak, S. Fremy, S. Kawai, T. Glatzel, H.J. Fang, L.A. Fendt, F. Diederich, E. Meyer: Directed rotations of single porphyrin molecules controlled by localized force spectroscopy, ACS Nano 6, 63186324 (2012)

24.238 S. Kawai, A. Benassi, E. Gnecco, H. Soede, R. Pawlak, X. Feng, K. Muellen, D. Passerone, C.A. Pignedoli, P. Ruffieux, R. Fasel, E. Meyer: Superlubricity of graphene nanoribbons on gold surfaces, Science 351, 957-961 (2016)

24.239 M. Nonnenmacher, M.P. Oboyle, H.K. Wickramasinghe: Kelvin probe force microscopy, Appl. Phys. Lett. 58, 2921-2923 (1991)

24.240 S. Sadewasser, T. Glatzel: Kelvin Probe Force Microscopy (Springer, Berlin, Heidelberg 2012)

24.241 T. König, G.H. Simon, H.P. Rust, M. Heyde: Work function measurements of thin oxide films on metals-Mg0 on $\mathrm{Ag}(001), \mathrm{J}$. Phys. Chem. C 113, 11301-11305 (2009)

24.242 K. Moloni, B.M. Moskowitz, E.D. Dahlberg: Domain structures in single crystal magnetite below the verwey transition as observed with a lowtemperature magnetic force microscope, Geophys. Res. Lett. 23, 2851-2854 (1996)

24.243 M. Liebmann, U. Kaiser, A. Schwarz, R. Wiesendanger, U.H. Pi, T.W. Noh, Z.G. Khim, D.W. Kim: Domain nucleation and growth of $\mathrm{La}_{0.7} \mathrm{Ca}_{0.3} \mathrm{MnO}_{3-\delta} / \mathrm{LaAlO}_{3}$ films studied by low temperature magnetic force microscopy, J. Appl. Phys. 93, 8319-8321 (2003)

24.244 Q.Y. Lu, C.C. Chen, A. de Lozanne: Observation of magnetic domain behavior in colossal magnetoresistive materials with a magnetic force microscope, Science 276, 2006-2008 (1997)

24.245 G.M. Xiao, J.H. Ross, A. Parasiris, K.D.D. Rathnayaka, D.G. Naugle: Low-temperature MFM studies of CMR manganites, Physica C 341, 769-770 (2000)

24.246 A. Moser, H.J. Hug, I. Parashikov, B. Stiefel, 0. Fritz, H. Thomas, K. Baratoff, H.J. Guntherodt P. Chaudhari: Observation of single vortices condensed into a vortex-glass phase by magnetic force microscopy, Phys. Rev. Lett. 74, 1847-1850 (1995) 
24.247 U.H. Pi, D.H. Kim, Z.G. Khim, U. Kaiser, M. Liebmann, A. Schwarz, R. Wiesendanger: Vortex dynamics in $\mathrm{Bi}_{2} \mathrm{Sr}_{2} \mathrm{CaCu}_{2} \mathrm{O}_{8}$ single crystal with low density columnar defects studied by magnetic force microscope, J. Low Temp. Phys. 131, 9931002 (2003)

24.248 M. Roseman, P. Grutter: Estimating the magnetic penetration depth using constant-height magnetic force microscopy images of vortices, New J. Phys. 3, 241-248 (2001)

24.249 M. Roseman, P. Grutter, A. Badia, V. Metlushko: Flux lattice imaging of a patterned niobium thin film, J. Appl. Phys. 89, 6787-6789 (2001)

24.250 A. Volodin, K. Temst, C. Van Haesendonck, Y. Bruynseraede: Imaging of vortices in conventional superconductors by magnetic force $\mathrm{mi}-$ croscopy, Physica C 332, 156-159 (2000)

24.251 A. Volodin, K. Temst, C. Van Haesendonck, Y. Bruynseraede, M.I. Montero, I.K. Schuller: Magnetic-force microscopy of vortices in thin niobium films: Correlation between the vortex distribution and the thickness-dependent film morphology, Europhys. Lett. 58, 582-588 (2002)

24.252 C.W. Yuan, Z. Zheng, A.L. de Lozanne, M. Tortonese, D.A. Rudman, J.N. Eckstein: Vortex images in thin films of $\mathrm{YBa}_{2} \mathrm{Cu}_{3} \mathrm{O}_{7-x}$ and $\mathrm{Bi}_{2} \mathrm{Sr}_{2} \mathrm{CaCu}_{2} \mathrm{O}_{8+x}$ obtained by low-temperature magnetic force $\mathrm{mi}-$ croscopy, J. Vac. Sci. Technol. B 14, 1210-1213 (1996)

24.253 A. Moser, H.J. Hug, B. Stiefel, H.J. Guntherodt: Low temperature magnetic force microscopy on $\mathrm{YBa}_{2} \mathrm{Cu}_{3} \mathrm{O}_{7-\delta}$ thin films, J. Magn. Magn. Mater. 190, 114-123 (1998)

24.254 A. Volodin, K. Temst, C. Van Haesendonck, Y. Bruynseraede: Observation of the Abrikosov vortex lattice in $\mathrm{NbSe}_{2}$ with magnetic force microscopy, Appl. Phys. Lett. 73, 1134-1136 (1998)

24.255 A. Schwarz, R. Wiesendanger: Magnetic sensitive force microscopy, Nano Today 3, 28-39 (2008)

24.256 R. Wiesendanger, D. Burgler, G. Tarrach, A. Wadas, D. Brodbeck, H.J. Guntherodt, G. Guntherodt, R.J. Gambino, R. Ruf: Vacuum tunneling of spinpolarized electrons detected by scanning tunneling microscopy, J. Vac. Sci. Technol. B 9, 519-524 (1991)

24.257 H. Momida, T. Oguchi: First-principles study on exchange force image of $\mathrm{NiO}(001)$ surface using a ferromagnetic Fe probe, Surf. Sci. 590, 42-50 (2005)

24.258 U. Kaiser, A. Schwarz, R. Wiesendanger: Magnetic exchange force microscopy with atomic resolution, Nature 446, 522-525 (2007)

24.259 R. Schmidt, C. Lazo, U. Kaiser, A. Schwarz, S. Heinze, R. Wiesendanger: Quantitative measurement of the magnetic exchange interaction across a vacuum gap, Phys. Rev. Lett. 106, 257202 (2011)

24.260 R. Schmidt, C. Lazo, H. Holscher, U.H. Pi, V. Caciuc, A. Schwarz, R. Wiesendanger, S. Heinze: Probing the magnetic exchange forces of iron on the atomic scale, Nano Lett. 9, 200-204 (2008)

24.261 R. Schmidt, A. Schwarz, R. Wiesendanger: Magnetization switching utilizing the magnetic exchange interaction, Phys. Rev. B 86, 174402 (2012)

24.262 J.A. Sidles, J.L. Garbini, K.J. Bruland, D. Rugar, 0. Zuger, S. Hoen, C.S. Yannoni: Magnetic resonance force microscopy, Rev. Modern Phys. 67, 249-265 (1995)

24.263 J.A. Sidles, J.L. Garbini, G.P. Drobny: The theory of oscillator-coupled magnetic resonance with potential applications to molecular imaging, Rev. Sci. Instrum. 63, 3881-3899 (1992)

24.264 D. Rugar, R. Budakian, H.J. Mamin, B.W. Chui: Single spin detection by magnetic resonance force microscopy, Nature 430, 329-332 (2004)

24.265 H.J. Mamin, D. Rugar: Sub-attonewton force detection at millikelvin temperatures, Appl. Phys. Lett. 79, 3358-3360 (2001)

24.266 D. Rugar, 0. Zuger, S. Hoen, C.S. Yannoni, H.M. Vieth, R.D. Kendrick: Force detection of nuclear magnetic resonance, Science 264, 1560-1563 (1994)

24.267 Z. Zhang, P.C. Hammel, P.E. Wigen: Observation of ferromagnetic resonance in a microscopic sample using magnetic resonance force microscopy, Appl. Phys. Lett. 68, 2005-2007 (1996)

24.268 C.L. Degen, M. Poggio, H.J. Mamin, C.T. Rettner, D. Rugar: Nanoscale magnetic resonance imaging, Proc. Natl. Acad. Sci. U.S.A. 106, 1313-1317 (2009)

24.269 R.P. Feynman: There is plenty of room at the bottom, Eng. Sci. 23, 22-25 (1960) 